Article

\title{
Synthesis of Pyrrolidine Monocyclic Analogues of Pochonicine and Its Stereoisomers: Pursuit of Simplified Structures and Potent $\beta-N$-Acetylhexosaminidase Inhibition
}

\author{
Xin Yan ${ }^{1,2}$, Yuna Shimadate ${ }^{3}$, Atsushi Kato ${ }^{3, *}$ (]) Yi-Xian Li ${ }^{1,2, *}$, Yue-Mei Jia ${ }^{1,2}$, \\ George W. J. Fleet ${ }^{4,5}$ and Chu-Yi Yu ${ }^{1,2,5, *(D)}$ \\ 1 Beijing National Laboratory for Molecular Science (BNLMS), CAS Key Laboratory of Molecular Recognition \\ and Function, Institute of Chemistry, Chinese Academy of Sciences, Beijing 100190, China; \\ yanxin2012@iccas.ac.cn (X.Y.); jiayuemei@iccas.ac.cn (Y.-M.J.) \\ 2 University of Chinese Academy of Sciences, Beijing 100049, China \\ 3 Department of Hospital Pharmacy, University of Toyama, 2630 Sugitani, Toyama 930-0194, Japan; \\ m1961224@ems.u-toyama.ac.jp \\ 4 Chemistry Research Laboratory, Department of Chemistry, University of Oxford, Mansfield Road, \\ Oxford OX13TA, UK; george.fleet@chem.ox.ac.uk \\ 5 National Engineering Research Center for Carbohydrate Synthesis, Jiangxi Normal University, \\ Nanchang 330022, China \\ * Correspondence: kato@med.u-toyama.ac.jp (A.K.); tamarali@iccas.ac.cn (Y.-X.L.); yucy@iccas.ac.cn (C.-Y.Y.); \\ Tel.: +86-010-626-12893 (C.-Y.Y.)
}

Academic Editors: Ramón J. Estévez Cabanas, George Fleet, Atsushi Kato and Robert J. Nash Received: 21 February 2020; Accepted: 23 March 2020; Published: 25 March 2020

\begin{abstract}
Ten pairs of pyrrolidine analogues of pochonicine and its stereoisomers have been synthesized from four enantiomeric pairs of polyhydroxylated cyclic nitrones. Among the ten $N$-acetylamino pyrrolidine analogues, only compounds with 2,5-dideoxy-2,5-imino-D-mannitol (DMDP) and pochonicine (1) configurations showed potent inhibition of $\beta-N$-acetylhexosaminidases ( $\beta$-HexNAcases); while 1-amino analogues lost almost all their inhibitions towards the tested enzymes. The assay results reveal the importance of the $N$-acetylamino group and the possible right configurations of pyrrolidine ring required for this type of inhibitors.
\end{abstract}

Keywords: Pochonicine; pharmacophore; synthesis; $\beta$-N-acetylhexosaminidase; iminosugars; structure-activity relationship

\section{Introduction}

Since its isolation from fungus Pochonia suchlasporia var. suchlasporia TAMA 87 in 2009 [1], pochonicine (1) (Figure 1) has been an attractive synthetic target due to its potent and specific inhibition of $\beta$ - $N$-acetylhexosaminidases ( $\beta$-HexNAcases), including $\beta$ - $N$-acetylglucosaminidases ( $\beta$-GlcNAcases) and $\beta$ - $N$-acetylgalactosaminidases ( $\beta$-GalNAcases) $[1,2]$. $\beta$-HexNAcases are associated with many crucial biological processes [3]. In fungi and insects, the enzymes play important roles in the metabolism of the polysaccharide chitin [4,5]; in mammals, $\beta$-HexNAcases participate in the regulation of cell signalling and influence protein expression, degradation and trafficking [6]. In humans, many diseases including lysosomal storage disorders [7], type-II diabetes [6], insulin resistance [8] and Alzheimer's disease [9-11] can be attributed to abnormality of $\beta$-HexNAcases. Therefore, study of $\beta$-HexNAcase inhibitors may provide alternate strategies for discovery of therapeutic drugs. 

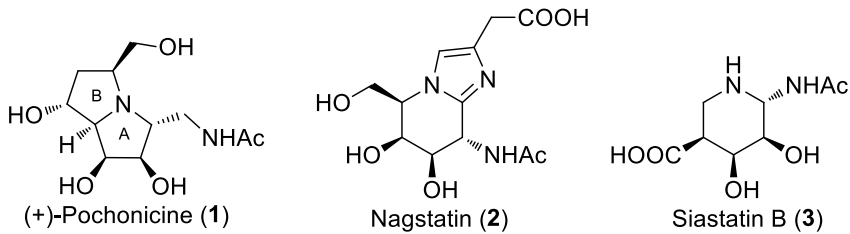

Figure 1. Naturally occurring potent $N$-acetylhexosaminidases (HexNAcase) inhibitors.

As one of the most potent $\beta$-HexNAcase inhibitors, pochonicine (1) possesses a polyhydroxylated pyrrolizidine skeleton, in contrast to the polyhydroxylated piperidine ring present in the other two naturally occurring iminosugars, nagstatin (2) [12] and siastatin B (3) (Figure 1) [13]. The $\mathrm{N}$-acetylamino group is the common structural feature that distinguishes these natural products from the other iminosugars [14]. A diverse range of potent synthetic $\beta$-HexNAcase iminosugar inhibitors have been reported including pyrrolidines (4 [15-17], 5 [18,19], 6 [20,21] and 7 [22]), piperidines (8 [23,24], 9 [25] and 10 [26]), azepanes (11 [27-29]) and azetidines (12 [30] and 13 [31]); almost all of them contain an $\mathrm{N}$-acetylamino group (Figure 2) [32].

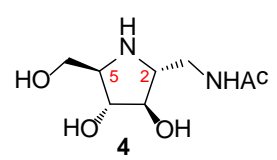

0:16 $\mu \mathrm{M}\left[{ }^{15}\right] ; 0$ 30 $\left.\mu \mathrm{M}^{16}\right]$<smiles>CN(C)C1CN[C@H](CO)[C@@H](O)[C@H]1O</smiles>

NOt determined

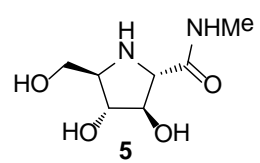

$\left.00^{\circ} 03 \mathrm{M}^{18}\right] ; 0$. $20 \mu \mathrm{M}^{18}$<smiles>CN[C@H]1[C@@H](CO)N[C@H](CO)[C@@H](O)[C@H]1O</smiles>

$\left.\left.41 \mu \mathrm{M}^{26}\right] ; 65 \mu \mathrm{M}^{26}\right]$

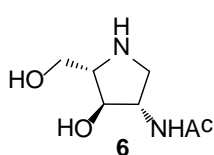

$\left.3.4 \mu \mathrm{M}^{20}\right] ; 0$ 64 $\left.\mu \mathrm{M}^{20}\right]$<smiles>CNC1CNCC(O)[C@H](O)[C@@H]1O</smiles>

$\left.\mathrm{Hex} A K_{\mathrm{i}}={ }_{3} 6 \mu \mathrm{M}^{29}\right]$

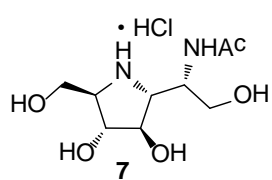

$\left.\left.3.4 \mu \mathrm{M}^{22}\right] ; 15 \mu \mathrm{M}^{[22}\right]$

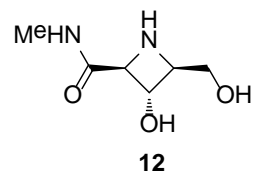

$\left.2.8 \mu \mathrm{M}^{30}\right] ; 3.3 \mu \mathrm{M}^{[30]}$;

$\left.14 \mu \mathrm{M}^{30}\right]$<smiles>CN(C)C1CNC(CO)C1O</smiles>

$\left.\left.2 \cdot 9 \mu \mathrm{M}^{24}\right] ; 70 \mu \mathrm{M}^{24}\right]$

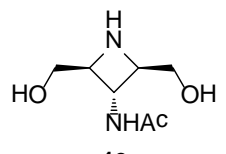

13

No inhibition[31]

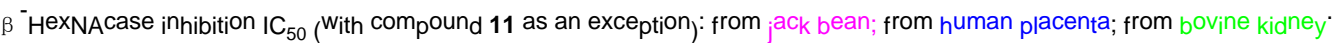

Figure 2. Examples of synthetic HexNAcase inhibitors.

The novel structure and inhibition properties of pochonicine (1) led to the rapid report of total syntheses [2], together with its enantiomer [33] and analogues [34]. However, syntheses of such highly substituted bicyclic pyrrolizidines with up to seven contiguous stereogenic centres are long and complex $[35,36]$. In contrast, construction of monocyclic pyrrolidine iminosugars with only four chiral centres are easier to accomplish. The pyrrolidine core of the corresponding iminosugar frequently appears to be the HexNAcase pharmacophore [37-39], as the pyrrolidine sections are responsible for mimicking the transition-state of enzyme reaction [40]. Among these monocyclic iminosugars, a series of pyrrolidines containing acetamide groups which are essential for their $\beta$-HexNAcase inhibitions were reported (Figure 2), among which considerable examples were accomplished before the isolation of pochonicine (1) [41,42]. Expectedly, quite a number of these five-membered acetamide derivatives were found to be potent $\beta$-HexNAcase inhibitors $[16,21,25,26]$. In continuation of our interests in structure-activity relationship study of iminosugars [39,43-46], in this work, 20 stereoisomeric pyrrolidine analogues of pochonicine (1) were synthesized and systematically assayed as glycosidase inhibitors, in order to look for novel molecules with simplified structure and remained potent inhibitory activities. 


\section{Results and Discussion}

\subsection{Synthesis of 1-N-Acetylamino-2,5-Imino-1,2,5-Trideoxy-L-Mannitol hydrochloride (A-10)}

Synthesis of acetamide modified pyrrolidines generally rely on asymmetric synthesis from achiral starting materials or begin with carbohydrate precursors [32]. In the second strategy, carbohydrate-derived nitrones have significant advantages due to their ready chirality, availability and versatile chemistry [47,48]. In this work, polyhydroxylated nitrones A-H (Figure 3) were readily prepared from the enantiomers of xylose, arabinose, lyxose and ribose by literature methods [16,49-51].
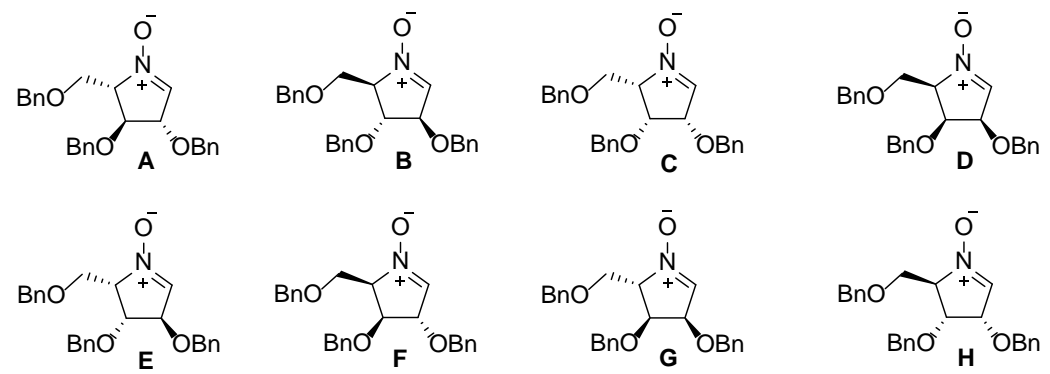

Figure 3. Polyhydroxylated nitrones used in building pyrrolidine cores.

The D-xylose-derived nitrone $\mathbf{A}$ is a convenient starting material for the synthesis of 1-amino-2,5-imino-1,2,5-trideoxy-L-mannitol hydrochloride (A-11) and its 1-N-acetylamino derivative (A-10) [16]. Nucleophilic additions with trimethylsilyl cyanide (TMSCN) [16] and nitromethane [52] were studied as agents for the introduction of aminomethyl substituents. Reaction of nitrone $\mathbf{A}$ with TMSCN at room temperature provide hydroxylamine A-2 in $96 \%$ yield as the sole product, and its C-2 configuration was determined as S-configuration through NOESY experiments since a strong interaction of $\mathrm{H}-2$ and $\mathrm{H}-4$ was observed; in contrast, the aza-Henry reaction gave a pair of inseparable epimers (A-2' $\mathbf{a}$ and $\left.\mathbf{A}-\mathbf{2}^{\prime} \mathbf{b}\right)$ in a 1:1 ratio. Since the corresponding reduction products were also difficult to purify, the attempt to introduce aminomethyl groups by aza-Henry reaction was not further investigated. The addition product A-2 was treated with Raney $\mathrm{Ni} / \mathrm{H}_{2}$ in the presence of $\mathrm{Boc}_{2} \mathrm{O}$, and then deprotected to afford diamine A-3 in good yield. Final hydrogenation of A-3 gave 1-amino-2,5-imino-1,2,5-trideoxy-L-mannitol dihydrochloride (A-11) in quantitative yield (Scheme 1).

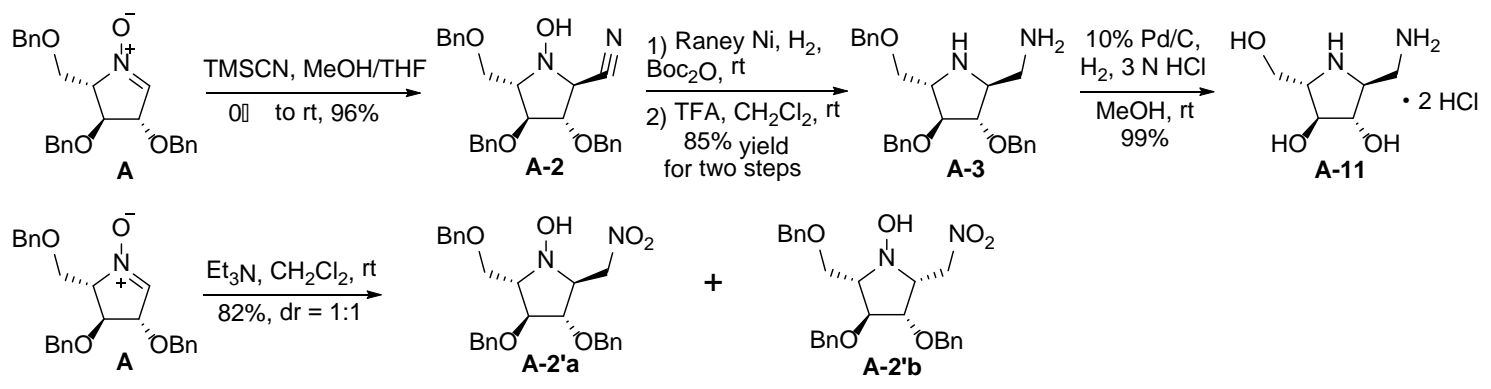

Scheme 1. Synthesis of compound A-11 from L-arabino-nitrone (A).

Treatment of diamine A-3 with acetic anhydride provided compound A-4 in high yield, but the attempt to release the secondary amine selectively by hydrochloric acid was unsatisfactory, giving the target product A-5 in only 19\% yield together with part of diamine A-3 recovered (Scheme 2). Selective reduction of the $\mathrm{N}-\mathrm{O}$ bond of hydroxylamine A-2, can be achieved in the presence of $\mathrm{SmI}_{2}$ according to the reported method in moderate yield [53]. Since excessive amount of $\mathrm{SmI}_{2}$ was needed in above step, other reduction conditions were also tried. Though the typical $\mathrm{Zn}-\mathrm{Cu}(\mathrm{OAc})_{2}-\mathrm{AcOH}$ system [16,54] led to complex mixtures, the modified condition with zinc replaced by iron [55] provided 
the target amine A-6 in excellent yield with no effect on the nitrile. Subsequent $N$-protection gave the carbamate A-7, which was then hydrogenated to convert the cyanide group to the primary amine. However, the reduction to amine A-8 proceeded in low yield (20-30\%). Though the remaining two steps, $N$-acetylation and deprotection both can go smoothly to afford their corresponding products, the unsatisfactory reduction yield of compound A-7 seriously reduced the total yield of the whole route. Selective acetylation of the primary amine A-3 was also tried by strictly control of the usage of acetic anhydride and reaction time [15,16], but no target product was obtained. Unexpectedly, acetylation with acetic acid in dichloromethane provided the monoacetylated compound A-5 in 18\% yield (improved to $24 \%$ yield when anhydrous $\mathrm{MgSO}_{4}$ was added). However, further attempts to improve the reaction were unsuccessful. In contrast, the mild acylation reagent $N, N^{\prime}, N^{\prime \prime}, N^{\prime \prime \prime}$-tetraacetylglycoluril [56] showed excellent selectivity for primary amine acetylation, giving the monoacetylated A-5 in $92 \%$ yield when refluxing together in dichloromethane. Final hydrogenation of the intermediate then furnished the target product A-10 quantitatively.

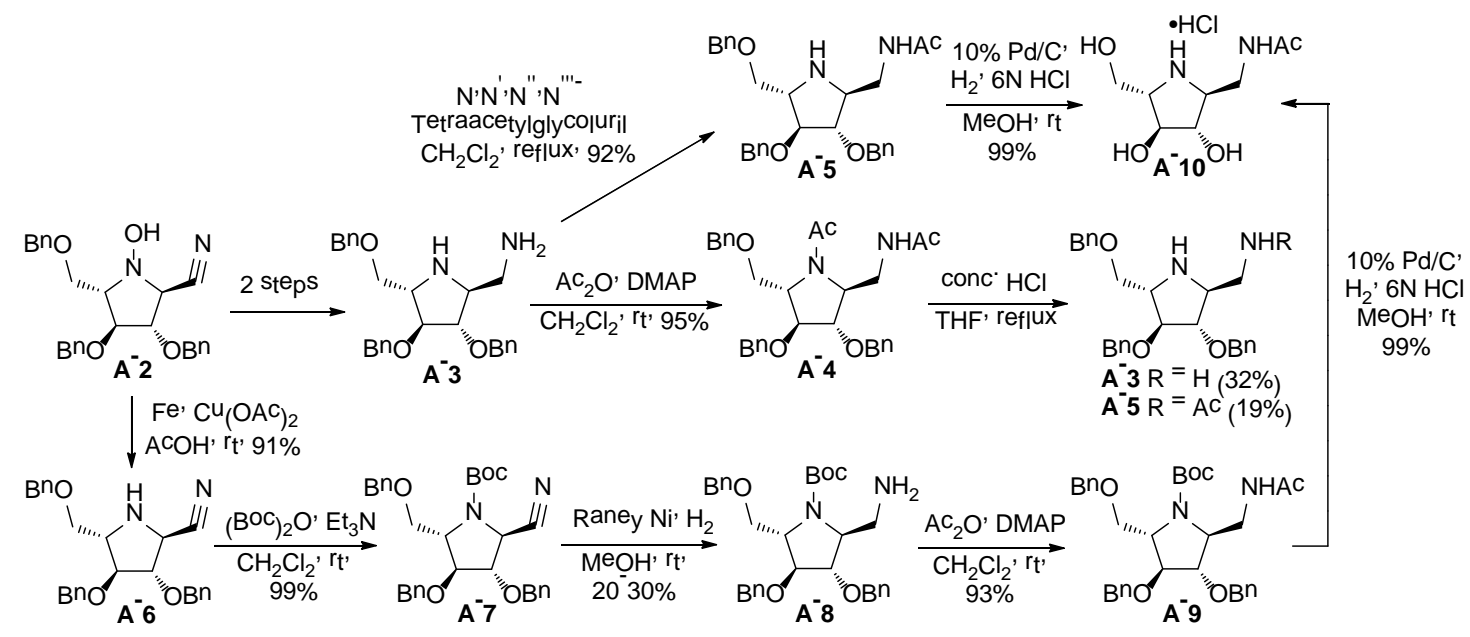

Scheme 2. Synthesis of monoacetylated compound A-10.

\subsection{Synthesis of 1-Amino and 1-N-Acetylamino Modified Pyrrolidine Stereoisomers}

According to the strategy in Schemes 1 and 2, other 18 stereoisomeric pyrrolidine analogues of pochonicine (1) were synthesized from the corresponding nitrones $\mathbf{B}-\mathbf{H}$. Reaction of TMSCN with nitrones gave exclusively trans-addition products in high yields, with nitrone $\mathbf{E}$ and $\mathbf{F}$ as exceptions. Cyanation of nitrone $\mathbf{E}$ and $\mathbf{F}$ both afforded a pair of diastereomers with trans/cis ratios as 63:37 and 61:39, respectively. The configurations of the newly constructed chiral centres in compound D-2, F-2b and H-2 were unambiguously confirmed by X-ray crystallographic analysis (See Supplementary Materials). The structures of their corresponding enantiomers C-2, E-2b and G-2 can also be confirmed since their NMR data were indeed identical. Reduction of the resulting hydroxylamines by Raney $\mathrm{Ni} / \mathrm{H}_{2}$ in the presence of $\mathrm{Boc}_{2} \mathrm{O}$ and subsequent deprotection afforded diamines $\mathbf{B}-\mathbf{3}-\mathbf{H}-\mathbf{3}$, which were then acetylated on the primary amine groups to give compounds $\mathbf{B}-\mathbf{5}-\mathbf{H}-\mathbf{5}$. Final hydrogenation of intermediates B-3-H-3 and B-5- $\mathbf{H}-\mathbf{5}$ provided the target products, i.e., $\mathbf{4} \cdot \mathbf{H C l}, \mathbf{C}-\mathbf{1 0}-\mathbf{H}-\mathbf{1 0}$ and B-11-H-11 (Table 1).

\subsection{Glycosidase Inhibition}

The synthesized 1-N-acetylamino and 1-amino pyrrolidine analogues were assayed against a range of enzymes, as shown in Tables 2 and 3.

Compound D-10 which resembles the pyrrolidine ring of pochonicine (1) exhibited potent inhibition of $\beta$-GlcNAcases from various resources including bovine liver, HL60 and Jack bean ( $\mathrm{IC}_{50}$ $2.8 \mu \mathrm{M}, 10 \mu \mathrm{M}$ and $0.12 \mu \mathrm{M}$, respectively). While compound $4 \cdot \mathbf{H C l}$, the 1 -deoxy-1-N-acetylamino 
derivative of 2,5-dideoxy-2,5-imino-D-mannitol (DMDP) [57], also behaved as similar potent inhibitor of bovine liver and Jack bean $\beta$-GlcNAcase ( $\mathrm{IC}_{50} 4.7 \mu \mathrm{M}$ and $0.21 \mu \mathrm{M}$, respectively). Both the two compounds were found to potent inhibitors of HL60 $\beta$-GalNAcase $\left(\mathrm{IC}_{50} 9.5 \mu \mathrm{M}\right.$ and $8.8 \mu \mathrm{M}$, respectively). However, comparing to the natural product pochonicine (1), the very powerful inhibitor of $\beta$-GlcNAcases and $\beta$-GalNAcases, both the two analogues showed significant decrease in the inhibition of two enzymes. Unexpectedly, pochonicine (1) did not exhibit any inhibition of $\alpha$-GalNAcases, but compound D-10 showed moderate inhibition of chicken liver $\alpha$-GalNAcase $\left(\mathrm{IC}_{50} 65.3 \mu \mathrm{M}\right)$. For other 1- $\mathrm{N}$-acetylamino compounds tested in Table 2, part of the compounds are only moderate or weak inhibition of the tested $\beta$-HexNAcases, and the other completely lost their $\beta$-HexNAcase inhibitory activities.

As shown in Table 3, 1-amino pyrrolidine analogues failed to provide positive assay results. Most of them showed weak or no inhibition of all the glycosidases tested. As an exception, compound D-11 with the pyrrolidine ring of pochonicine (1) were found to be moderate inhibitor of $\alpha$-mannosidase $\left(\mathrm{IC}_{50} 54 \mu \mathrm{M}\right)$ and $\beta$-GlcNAcase $\left(\mathrm{IC}_{50} 99 \mu \mathrm{M}\right)$ from Jack bean, and $\alpha$-GalNAcase ( $\left.\mathrm{IC}_{50} 44 \mu \mathrm{M}\right)$ from chicken liver. The assay results further indicated the importance of acetamide groups for $\beta$-HexNAcase inhibitors. However, the more potent inhibition of compound D-11 of $\alpha$-GalNAcase than compound D-10 may indicate the significant role of the scaffold in interaction with active sites of the enzyme instead of the 1- $N$-acetylamino group.

Though all the compounds in Tables 2 and 3 can also be regarded as $1-N$-acetylamino and 1 -amino derivatives of their corresponding pyrrolidines (for example, compound $4 \cdot \mathbf{H C l}$ is the 1-deoxy-1-Nacetylamino derivative of DMDP), they lost almost all their inhibition towards other tested glycosidases including glucosidase, galactosidase, mannosidase, $\alpha$-L-fucosidase, trehalase, amyloglucosidase, $\alpha$-L-rhamnosidase and $\beta$-glucuronidase, revealing the importance of C-1 hydroxyl groups in interaction with the corresponding enzymes.

Therefore, both configurations of the pyrrolidine ring and the 1-N-acetylamino group have significant influences on the inhibition of $\beta$-HexNAcases and $\alpha$-GalNAcase. In detail, $1-N$-acetylamino pyrrolidine analogues with the same configuration as DMDP and pochonicine (1) showed powerful inhibition of these enzymes, revealing the importance of the right configurations of A ring. Furthermore, the results indicate that pochonicine analogue with the A ring in DMDP configuration may also turn out to be potent inhibitors of the above enzymes. The structure-activity relationship reported in this work may be helpful in pursuing simplified pochonicine (1) analogues and more potent glycosidase inhibitors. 
Table 1. 1-Amino and 1-N-acetylamino pyrrolidine analogues synthesized from cyclic nitrones.

\begin{tabular}{|c|c|c|c|c|c|c|}
\hline Entry & Cyclic Nitrone & Hydroxylamine & Diamine & Monoacetylated Pyrrolidine & 1-Amino Product & 1-N-Acetylamino Product \\
\hline 1 & $\begin{array}{c}\text { Bno } \\
\text { Larabino-nitrone } \\
\text { (A) }\end{array}$ & $\begin{array}{c}\mathrm{Bn} \mathrm{A}^{\mathrm{B}-\mathrm{OBn}} \\
\left.(96 \%)^{\mathrm{a}}\right)^{\mathrm{a}}\end{array}$ & 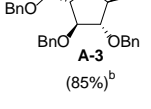 & $\begin{array}{c}\mathrm{BnO} \\
\mathrm{A}-5 \\
(88 \%))^{\mathrm{O}} \mathrm{Bn}\end{array}$ & $\begin{array}{c}\mathrm{HO} \mathrm{A}-11^{\mathrm{OH}} \\
(99 \%)^{\mathrm{d}}\end{array}$ & ${ }_{(99 \%)^{e}}$ \\
\hline 2 & 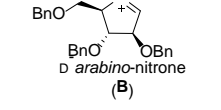 & $\begin{array}{c}\mathrm{BnO} \\
(94 \%)\end{array}$ & $\begin{array}{c}\mathrm{BnO} \\
(88 \%)\end{array}$ & $\begin{array}{c}\mathrm{Bno}{ }_{\mathrm{B}-5} \mathrm{OB} \\
(91 \%)\end{array}$ & 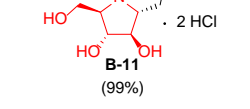 & $\begin{array}{l}\mathrm{OH}_{4} \cdot \mathrm{HCl} \\
(99 \%) \\
(\mathrm{HCl})\end{array}$ \\
\hline 3 & 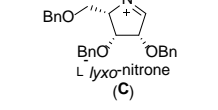 & $\begin{array}{c}\mathrm{Bno} \\
\mathrm{C}-2 \mathrm{Bn} \\
(96 \%)\end{array}$ & $\begin{array}{c}\mathrm{BnO}{ }_{C-3} \mathrm{OBn} \\
(86 \%)\end{array}$ & $\begin{array}{c}-5 \\
(85 \%)\end{array}$ & $\begin{array}{c}\mathrm{HO} \mathrm{C}^{\mathrm{C}-11} \\
(99 \%)\end{array}$ & $\begin{array}{c}\mathrm{HO} \\
\mathrm{C}-10 \\
(99 \%)\end{array}$ \\
\hline 4 & 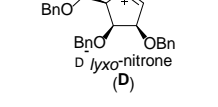 & $\begin{array}{c}\mathrm{Bn} \mathrm{D}_{\mathrm{D}-2} \mathrm{OBn} \\
(92 \%)\end{array}$ & $\begin{array}{c}\mathrm{D}-3 \\
(89 \%)\end{array}$ & $\begin{array}{c}5-5 \\
(82 \%)\end{array}$ & $\begin{array}{c}{ }^{\mathrm{H}} \mathrm{O}_{-11}^{\mathrm{OH}} \\
(999 \%)\end{array}$ & $\begin{array}{l}\text { D-10 } \\
(99 \%)\end{array}$ \\
\hline \multirow{2}{*}{5} & & & $\begin{array}{r}\mathrm{BnO}_{\mathrm{E}-3 \mathrm{a}}^{\mathrm{O}} \\
(88 \%))\end{array}$ & $\begin{array}{l}\text { E-5a } \\
(90 \%)\end{array}$ & $(99 \%)$ & $\begin{array}{l}\text { E-10a } \\
(99 \%)\end{array}$ \\
\hline & (E) & 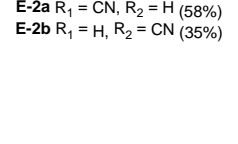 & 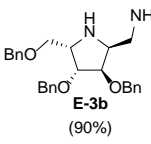 & $\begin{array}{l}\text { E-55 } \\
(82 \%)\end{array}$ & (9.116) & $\begin{array}{l}\mathrm{E}^{-10 \mathrm{~b}} \\
(99 \%)\end{array}$ \\
\hline \multirow{2}{*}{6} & 27 & 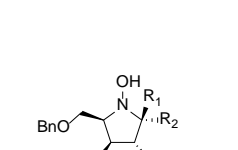 & $\begin{array}{r}\mathrm{F}-3 \mathrm{a} \\
(83 \%)\end{array}$ & $\begin{array}{c}\mathrm{BnO}-5 \mathrm{a} n \\
(87 \%)\end{array}$ & $\begin{array}{c}\mathrm{HO}=\mathrm{OH} \\
\begin{array}{c}\mathrm{E}-11 \mathrm{OH} \\
(99 \%)\end{array}\end{array}$ & $\begin{array}{l}\mathrm{F}_{\mathrm{F}-10 \mathrm{OH}}^{\mathrm{OH}} \\
(99 \%)\end{array}$ \\
\hline & $\begin{array}{l}\text { Bny } \\
\text { Dxylonitrone } \\
\text { (F) }\end{array}$ & $\begin{array}{l}\mathrm{F}-2 \mathrm{a} \mathrm{R}_{1} \mathrm{CN}_{1}, \mathrm{R}_{2}=\mathrm{H}(59 \%) \\
\mathrm{F}-2 \mathrm{~b} \mathrm{R}_{1}=\mathrm{H}, \mathrm{R}_{2}=\mathrm{CN}(37 \% \%)\end{array}$ & $\underbrace{\mathrm{OBn}}_{\substack{\mathrm{F}-3 \mathrm{~b} \\
(85 \%)}}$ & $\begin{array}{c}\mathrm{Bnd}_{\mathrm{F}-5 \mathrm{~b}} \mathrm{oBn} \\
(83 \%)\end{array}$ & 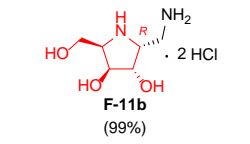 & $\begin{array}{l}\mathrm{Fo}_{\mathrm{F}}^{\mathrm{H}} \mathrm{ob} \\
(990 \%)\end{array}$ \\
\hline
\end{tabular}


Table 1. Cont

\begin{tabular}{|c|c|c|c|c|c|c|}
\hline Entry & Cyclic Nitrone & Hydroxylamine & Diamine & Monoacetylated Pyrrolidine & 1-Amino Product & 1-N-Acetylamino Product \\
\hline 7 & $\underbrace{\substack{\mathrm{O} \\
N}}_{\substack{\text { Bno } \\
\text { Liibo-nitrone } \\
(\mathbf{G})}}$ & $\begin{array}{c}\text { Bnd G-2 } \\
(87 \%) \\
\text { OBn }\end{array}$ & 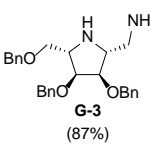 & $(86 \%)$ & 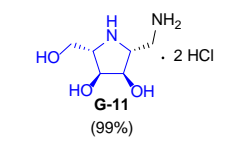 & $\sum_{\substack{\mathrm{G}-10 \\
(99 \%)}}^{\mathrm{OH}}$ \\
\hline 8 & 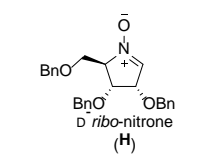 & $\begin{array}{c}\mathrm{Bno \sigma} \\
(91 \%)\end{array}$ & $\begin{array}{c}\mathrm{BnO} \\
\begin{array}{c}\mathrm{H}-3 \\
(88 \%)\end{array}\end{array}$ & $\begin{array}{c}\mathrm{Bnoc} \\
{ }_{\text {H-5 }} \mathrm{OBn} \\
(81 \%)\end{array}$ & 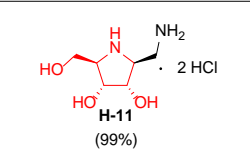 & . \\
\hline
\end{tabular}

${ }^{\text {a }}$ Yield of the corresponding hydroxylamines starting from cyclic nitrones; ${ }^{\mathrm{b}}$ Total yield in 2 steps starting from hydroxylamines to diamines; ${ }^{\mathrm{c}}$ Yield of monoacetylated pyrrolidines from the corresponding diamines; ${ }^{\mathrm{d}}$ Hydrogenation yield of 1 -amino products from diamines; ${ }^{\mathrm{e}}$ Hydrogenation yield of 1 - $N$-acetylamino products from the corresponding monoacetylated pyrrolidines.

Table 2. Concentrations of 1- $N$-acetylamino pyrrolidine analogues giving $50 \%$ inhibition of various enzymes.

\begin{tabular}{|c|c|c|c|c|c|c|c|c|c|c|c|}
\hline \multirow{3}{*}{ Enzyme } & \multicolumn{11}{|c|}{$\mathrm{IC}_{50}(\mu \mathrm{M})$} \\
\hline & & & & & ᄀ". $\mathrm{HCl}$ & & & & & & \\
\hline & A-10 & $4 \cdot \mathrm{HCl}$ & C-10 & D-10 & E-10a & F-10a & E-10b & F-10b & G-10 & H-10 & Pochonicine (1) \\
\hline \multicolumn{12}{|l|}{$\alpha$-Glucosidase } \\
\hline Yeast & $\mathrm{NI}^{\mathrm{a}}(0.5 \%)^{\mathrm{b}}$ & 450 & NI $(0 \%)$ & NI $(0 \%)$ & NI $(0 \%)$ & NI (6.91\%) & NI $(0 \%)$ & NI $(0 \%)$ & NI $(0 \%)$ & NI $(0 \%)$ & —c $^{c}$ \\
\hline Rice & 568 & 1000 & NI (18.2\%) & NI (26.1\%) & NI (15.9\%) & NI $(12.5 \%)$ & NI $(0 \%)$ & NI $(0 \%)$ & $\mathrm{NI}(0 \%)$ & NI (5.6\%) & - \\
\hline Rat intestinal maltase & 92 & 211 & NI (35.4\%) & NI (9.24\%) & NI (12.7\%) & NI (14.6\%) & NI $(0 \%)$ & NI (12.7\%) & NI $(16.0 \%)$ & NI (13.6\%) & - \\
\hline \multicolumn{12}{|l|}{$\beta$-Glucosidase } \\
\hline Almond & NI (16.1\%) & 170 & NI $(0 \%)$ & NI $(41.0 \%)$ & NI (37.9\%) & NI (11.6\%) & NI (1.2\%) & NI (19.8\%) & NI (15.3\%) & NI (17.1\%) & - \\
\hline Bovine liver & $\mathrm{NI}(2.0 \%)$ & $\mathrm{NI}(33.8 \%)$ & NI (1.0\%) & NI (2.4\%) & NI (4.1\%) & NI $(0.3 \%)$ & NI $(0 \%)$ & NI $(0 \%)$ & NI $(0 \%)$ & NI (1.7\%) & - \\
\hline \multicolumn{12}{|l|}{$\alpha$-Galactosidase } \\
\hline Coffee beans & NI $(0 \%)$ & NI (1.1\%) & NI $(0.36 \%)$ & 380 & NI $(0.36 \%)$ & NI $(0.36 \%)$ & NI $(0 \%)$ & $\mathrm{NI}(0 \%)$ & $\mathrm{NI}(0 \%)$ & NI $(0 \%)$ & - \\
\hline $\begin{array}{l}\text { - } \text {-Gavactosialine liver } \\
\alpha \text {-Mannosidase }\end{array}$ & NI (1.6\%) & 511 & NI (1.3\%) & NI (3.3\%) & NI (4.9\%) & NI (4.2\%) & NI (3.9\%) & NI (4.2\%) & NI (2.9\%) & NI (3.6\%) & - \\
\hline $\begin{array}{l}\alpha \text {-Mannosidase } \\
\text { Jack bean } \\
\beta \text {-Mannosidase }\end{array}$ & NI $(0 \%)$ & NI $(0 \%)$ & NI $(0.4 \%)$ & 205 & NI (0.7\%) & $\mathrm{NI}(0 \%)$ & NI (3.8\%) & NI (1.6\%) & NI (4.0\%) & NI (9.5\%) & - \\
\hline $\begin{array}{l}\text { Snail } \\
\alpha \text {-L-Fucosidase }\end{array}$ & NI $(0 \%)$ & 296 & $\mathrm{NI}(0 \%)$ & NI $(0 \%)$ & NI (25.8\%) & NI (3.23\%) & NI $(0 \%)$ & NI $(0 \%)$ & NI $(0 \%)$ & NI $(0 \%)$ & - \\
\hline Bovine kidney & $\mathrm{NI}(0 \%)$ & NI (1.5\%) & NI (4.9\%) & NI (1.7\%) & NI (41.7\%) & NI (8.4\%) & NI (20.5\%) & NI (3.9\%) & NI (11.5\%) & NI (22.3\%) & - \\
\hline
\end{tabular}


Table 2. Cont.

\begin{tabular}{|c|c|c|c|c|c|c|c|c|c|c|c|}
\hline \multirow{3}{*}{ Enzyme } & \multicolumn{11}{|c|}{$\mathrm{IC}_{50}(\mu \mathrm{M})$} \\
\hline & & & & & & & & & & & \\
\hline & A-10 & $4 \cdot \mathrm{HCl}$ & $\mathrm{C}-10$ & D-10 & E-10a & F-10a & $\mathrm{E}-10 \mathrm{~b}$ & F-10b & G-10 & H-10 & Pochonicine (1) \\
\hline \multicolumn{12}{|l|}{$\alpha, \alpha$-Trehalase } \\
\hline \multirow{2}{*}{\multicolumn{12}{|c|}{ Amyloglucosidase }} \\
\hline & & & & & & & & & & & \\
\hline A. niger & $\mathrm{NI}(5.5 \%)$ & NI $(40.6 \%)$ & NI $(1.9 \%)$ & $\mathrm{NI}(3.8 \%)$ & NI (4.0\%) & NI $(2.8 \%)$ & $\mathrm{NI}(6.4 \%)$ & NI (5.7\%) & NI (7.6\%) & $\mathrm{NI}(8.3 \%)$ & - \\
\hline \multicolumn{12}{|l|}{$\alpha$-L-Rhamnosidase } \\
\hline P. decumbens & NI (10.1\%) & $\mathrm{NI}(6.5 \%)$ & NI $(3.6 \%)$ & NI $(8.3 \%)$ & NI $(11.8 \%)$ & NI (5.3\%) & NI $(30.8 \%)$ & NI $(3.0 \%)$ & NI $(9.5 \%)$ & NI $(7.7 \%)$ & - \\
\hline \multicolumn{12}{|l|}{$\beta$-Glucuronidase } \\
\hline E. coli & NI $(0 \%)$ & NI $(0.6 \%)$ & NI $(0.3 \%)$ & NI $(0 \%)$ & NI $(0 \%)$ & NI $(0 \%)$ & NI $(0 \%)$ & NI $(0 \%)$ & NI $(0 \%)$ & NI $(0 \%)$ & - \\
\hline Bovine liver & $\mathrm{NI}(4.6 \%)$ & NI (1.7\%) & NI $(0 \%)$ & NI $(1.3 \%)$ & NI $(0 \%)$ & NI $(0 \%)$ & NI (4.3\%) & NI $(0 \%)$ & NI $(0 \%)$ & NI $(0 \%)$ & - \\
\hline \multicolumn{12}{|l|}{$\beta-N$ Acetylhexosaminidase } \\
\hline Bovine liver & 943 & 4.7 & NI $(36.3 \%)$ & 2.8 & 95 & NI $(23.2 \%)$ & NI (38.4\%) & 652 & 299 & NI (33.7\%) & 0.021 [2] \\
\hline HL60 & NI (12.7\%) & 34 & NI $(3.9 \%)$ & 10 & 591 & NI $(0 \%)$ & NI $(4.5 \%)$ & NI $(16.6 \%)$ & NI $(18.8 \%)$ & NI $(0.3 \%)$ & 0.018 [2] \\
\hline Jack bean & 202 & 0.21 & 129 & 0.12 & 10 & NI (42\%) & 115 & 98 & 26 & 241 & $0.0016[2]$ \\
\hline A. oryzae & - & - & - & - & - & - & - & - & - & - & $0.33[2]$ \\
\hline Human placenta & - & - & - & - & - & - & - & - & - & - & 0.012 [2] \\
\hline \multicolumn{12}{|l|}{$\beta-N$-Acetylgalactosaminidase } \\
\hline HL60 & NI (13.0\%) & 9.5 & NI (1.4\%) & 8.8 & 490 & NI $(0.9 \%)$ & NI (10.1\%) & NI (36.1\%) & NI $(15.9 \%)$ & NI (5.3\%) & 0.049 [2] \\
\hline$\alpha-N$-Acetylgalactosaminidase & & & & & & & & & & & \\
\hline Chicken liver & NI $(0 \%)$ & NI (3.3\%) & NI (6.1\%) & 65.3 & NI $(1.4 \%)$ & NI $(0 \%)$ & NI $(0.5 \%)$ & NI $(2.3 \%)$ & NI $(2.3 \%)$ & NI (6.1\%) & $\mathrm{NI}(9.0 \%)[2]$ \\
\hline
\end{tabular}

Table 3. Concentrations of 1-amino pyrrolidine analogues giving $50 \%$ inhibition of various enzymes.

\begin{tabular}{|c|c|c|c|c|c|c|c|c|c|c|}
\hline \multirow{3}{*}{ Enzyme } & \multicolumn{10}{|c|}{$\mathrm{IC}_{50}(\mu \mathrm{M})$} \\
\hline & $n^{H} \int^{N H}$ & & & & & & 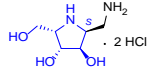 & ${ }_{\mathrm{HO}} \overbrace{\mathrm{HO}}^{\mathrm{N}} \overbrace{0}^{\mathrm{R}}$ & $\stackrel{H}{\mathrm{~N}}$ & ${ }_{\mathrm{Ho}} \overbrace{\mathrm{HO}}^{\mathrm{H}} \gamma^{\mathrm{N}}$ \\
\hline & A-11 & B-11 & C-11 & D-11 & E-11 & F-11a & E-11b & F-11b & G-11 & H-11 \\
\hline \multicolumn{11}{|l|}{$\alpha$-Glucosidase } \\
\hline Yeast & $\mathrm{NI}^{\mathrm{a}}(0 \%)^{\mathrm{b}}$ & NI (42.7\%) & NI (9.55\%) & NI $(4.52 \%)$ & NI (9.55\%) & NI (10.1\%) & NI $(0 \%)$ & NI $(0 \%)$ & $\mathrm{NI}(0 \%)$ & NI (0\%) \\
\hline Rice & 286 & 725 & NI $(0 \%)$ & NI $(8.4 \%)$ & NI $(15.7 \%)$ & NI (31.5\%) & NI $(12.9 \%)$ & NI $(16.9 \%)$ & NI $(11.8 \%)$ & NI $(3.4 \%)$ \\
\hline Rat intestinal maltase & 68 & 251 & NI (37.1\%) & $\mathrm{NI}(4.02 \%)$ & NI $(9.95 \%)$ & NI (48.0\%) & NI $(10.9 \%)$ & NI $(20.8 \%)$ & NI (\%2.64) & NI (10.1\%) \\
\hline$\beta$-Glucosidase & & & & & & & & & & \\
\hline
\end{tabular}


Table 3. Cont

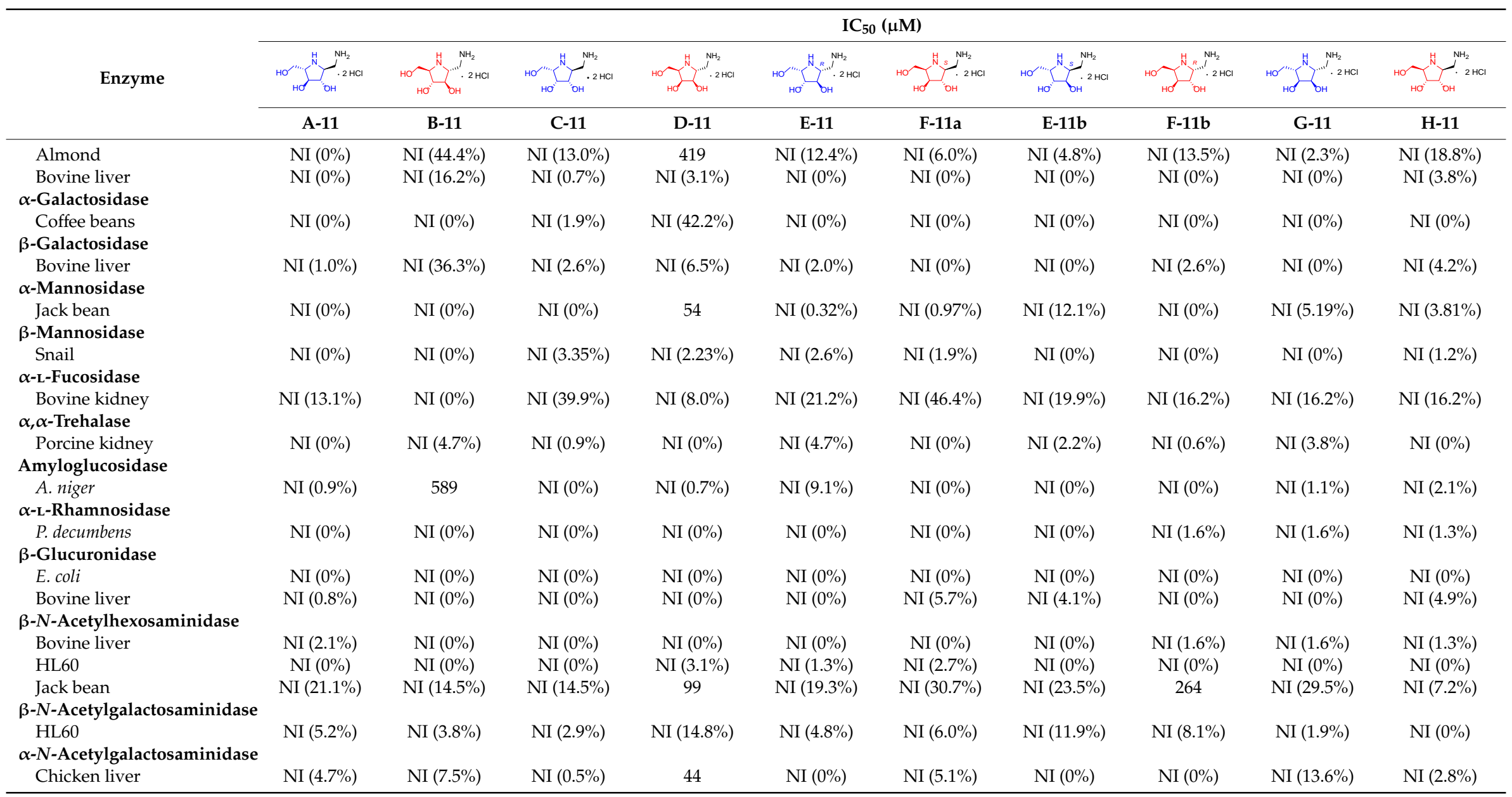

${ }^{a}$ NI: No Inhibition (less than $50 \%$ at $1000 \mu \mathrm{M}$ ); ${ }^{\text {b }}$ ( ): Inhibition $\%$ at $1000 \mu \mathrm{M}$. 


\section{Materials and Methods}

\subsection{General Methods}

All reagents were used as received without any further purification or prepared as described in the literature. TLC plates were visualized by ultraviolet light or by treatment with a spray of Pancaldi reagent $\left(\left(\mathrm{NH}_{4}\right)_{6} \mathrm{MoO}_{4}, \mathrm{Ce}\left(\mathrm{SO}_{4}\right)_{2}, \mathrm{H}_{2} \mathrm{SO}_{4}, \mathrm{H}_{2} \mathrm{O}\right)$ or a $0.5 \%$ solution of $\mathrm{KMnO}_{4}$ in acetone. Column chromatography was performed on a flash column chromatography with silica gel (200-300 mesh, Inno-chem, Beijing, China). NMR spectra were measured in $\mathrm{CDCl}_{3}$ (with TMS as internal standard) or $\mathrm{D}_{2} \mathrm{O}$ (with $\mathrm{H}_{2} \mathrm{O}$ as internal standard) on a Bruker AV300, AV400 or AV500 magnetic resonance spectrometer (Bruker, Ettlingen, Germany) $\left({ }^{1} \mathrm{H}\right.$ NMR at $300 \mathrm{MHz}, 400 \mathrm{MHz}$ or $500 \mathrm{MHz},{ }^{13} \mathrm{C} \mathrm{NMR}$ at $125 \mathrm{MHz}$ ). High-resolution mass spectra (HRMS) were performed on a Thermo Fisher Exactive Spectrometer (Thermo Fisher Scientific, Waltham, MA, USA). Polarimetry was determined using an Optical Activity AA-10R polarimeter with concentrations (c) given in gram per $100 \mathrm{~mL}$. Infrared spectra were recorded as films on KBr plates on a Nicolet-6700 FT-IR spectrometer (Thermo Fisher Scientific, Waltham, MA, USA).

\subsection{Material and Methods for the Enzyme Inhibition Assay}

With rat intestinal maltase as an exception, other enzymes were purchased from Sigma-Aldrich Chemical Co. (St. Louis, MO, USA). Brush border membranes prepared from rat small intestine according to the method of Kessler et al. [58] were assayed at pH 6.8 for rat intestinal maltase using maltose. The released D-glucose was determined colorimetrically using the Glucose CII-test Wako (Wako Pure Chemical Ind.; Osaka, Japan). Other glycosidase activities were determined using an appropriate $p$-nitrophenyl glycoside as substrate in a buffer solution at the optimal $\mathrm{pH}$ value of each enzyme. The reaction was stopped by adding $400 \mathrm{mM} \mathrm{Na} \mathrm{CO}_{3}$. The released $p$-nitrophenol was measured spectrometrically at $400 \mathrm{~nm}$ [59].

\subsection{Chemistry}

3.3.1. General Procedure for Synthesis of Hydroxylamines A-2, B-2, C-2, D-2, E-2a, E-2b, F-2a, F-2b, G-2 and H-2 with A-2 as an Example

To a solution of nitrone $\mathbf{A}(1.25 \mathrm{~g}, 3.00 \mathrm{mmol})$ in THF $(5 \mathrm{~mL})$ and methanol $(25 \mathrm{~mL})$ was added dropwise TMSCN $(0.45 \mathrm{~mL}, 3.60 \mathrm{mmol})$ under Ar atmosphere at $0{ }^{\circ} \mathrm{C}$. After stirring at room temperature for 6-8 h, TLC showed completion of the reaction. The resulting solution was concentrated in vacuo, and the residue was purified by flash column chromatography (silica gel, petroleum ether/EtOAc $=6 / 1$ ) to give hydroxylamine A-2 (colourless syrup, $1.28 \mathrm{~g}$, 96\% yield). Data for (2S,3S,4S,5S)-3,4-bis(benzyloxy)-5-(benzyloxymethyl)-2-cyano-1-hydroxypyrrolidine (A-2) (Ref. [16]): $[\alpha]_{\mathrm{D}}{ }^{23}-9.5$ (c 1.1 in $\left.\mathrm{CH}_{2} \mathrm{Cl}_{2}\right) ; v_{\max } / \mathrm{cm}^{-1}: 3366(\mathrm{~s}), 3088(\mathrm{~m}), 3063(\mathrm{~m}), 3030(\mathrm{~s}), 2920$ (s), 2867 (vs), 2237 (w), 1497 (s), 1454 (vs), 1362 (s), 1207 (m), 1100 (vs), 1028 (s), 738 (vs), 697 (vs); ${ }^{1} \mathrm{H} \mathrm{NMR}$ $\left(500 \mathrm{MHz}, \mathrm{CDCl}_{3}\right) \delta(\mathrm{ppm}): 7.36-7.20(\mathrm{~m}, 15 \mathrm{H}), 6.52(\mathrm{~s}, 1 \mathrm{H}, \mathrm{OH}), 4.54-4.37(\mathrm{~m}, 6 \mathrm{H}), 4.20(\mathrm{~d}, J=1.5 \mathrm{~Hz}$, $1 \mathrm{H}, \mathrm{H}-2), 4.13(\mathrm{t}, J=2.1 \mathrm{~Hz}, 1 \mathrm{H}, \mathrm{H}-3), 3.93(\mathrm{dd}, J=6.5$ and $2.2 \mathrm{~Hz}, 1 \mathrm{H}, \mathrm{H}-4), 3.72(\mathrm{dd}, J=10.5 \mathrm{and} 3.3 \mathrm{~Hz}$, $1 \mathrm{H}, \mathrm{H}-6), 3.54\left(\mathrm{dd}, J=10.5\right.$ and $\left.4.0 \mathrm{~Hz}, 1 \mathrm{H}, \mathrm{H}-6^{\prime}\right), 3.28-3.25(\mathrm{~m}, 1 \mathrm{H}, \mathrm{H}-5) ;{ }^{13} \mathrm{C} \mathrm{NMR}\left(125 \mathrm{MHz}, \mathrm{CDCl}_{3}\right) \delta$ (ppm): 137.4, 137.3, 136.4, 128.7, 128.4, 128.3, 128.0, 127.97, 127.93, 127.88, 127.82, 115.7, 83.6 (C-3), 81.2 (C-4), $73.3\left(\mathrm{PhCH}_{2}\right), 72.3\left(\mathrm{PhCH}_{2}\right), 72.0\left(\mathrm{PhCH}_{2}\right), 69.4$ (C-5), 66.4 (C-6), 61.2 (C-2); HRMS (ESI): calcd for $\mathrm{C}_{27} \mathrm{H}_{28} \mathrm{O}_{4} \mathrm{~N}_{2} \mathrm{Na}^{+}\left[\mathrm{M}+\mathrm{Na}^{+}\right]$467.1941, found 467.1941.

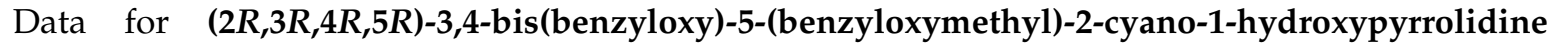
(B-2): Colourless syrup, $1.26 \mathrm{~g}, 94 \%$ yield from nitrone B $(1.26 \mathrm{~g}, 3.02 \mathrm{mmol}) ;[\alpha]_{\mathrm{D}}^{22}+8.7(c 1.0$ in $\left.\mathrm{CH}_{2} \mathrm{Cl}_{2}\right) ; v_{\text {max }} / \mathrm{cm}^{-1}$ : $3359(\mathrm{~m}), 3031(\mathrm{~m}), 2868(\mathrm{~m}), 2239(\mathrm{w}), 1496(\mathrm{~m}), 1454(\mathrm{~s}), 1362(\mathrm{~m}), 1207(\mathrm{~m})$, 1097 (vs), 1027 (m), 737 (s), 697 (vs); ${ }^{1} \mathrm{H}$ NMR (500 MHz, CDCl $\left.{ }_{3}\right) \delta(\mathrm{ppm}):$ 7.36-7.20 (m, 15H), 6.46 (s, 1H, OH), 4.55-4.37 (m, 6H), $4.20(\mathrm{~d}, \mathrm{~d}, J=1.5 \mathrm{~Hz}, 1 \mathrm{H}, \mathrm{H}-2), 4.14(\mathrm{t}, J=2.1 \mathrm{~Hz}, 1 \mathrm{H}, \mathrm{H}-3), 3.94(\mathrm{dd}$, $J=6.5$ and $2.2 \mathrm{~Hz}, 1 \mathrm{H}, \mathrm{H}-4), 3.72(\mathrm{dd}, J=10.5$ and $3.3 \mathrm{~Hz}, 1 \mathrm{H}, \mathrm{H}-6), 3.54(\mathrm{dd}, J=10.5$ and $4.0 \mathrm{~Hz}, 1 \mathrm{H}$, 
H-6' $), 3.28-3.26$ (m, 1H, H-5); ${ }^{13} \mathrm{C}$ NMR (125 MHz, CDCl $) \delta$ (ppm): 137.4, 137.3, 136.4, 128.7, 128.4, 128.3, 128.0, 127.96, 127.93, 127.87, 127.82, 115.7, 83.6 (C-3), $81.2(\mathrm{C}-4), 73.3\left(\mathrm{PhCH}_{2}\right), 72.3\left(\mathrm{PhCH}_{2}\right), 72.0$ $\left(\mathrm{PhCH}_{2}\right), 69.4$ (C-5), 66.4 (C-6), 61.1 (C-2); HRMS (ESI): calcd for $\mathrm{C}_{27} \mathrm{H}_{28} \mathrm{O}_{4} \mathrm{~N}_{2} \mathrm{Na}^{+}\left[\mathrm{M}+\mathrm{Na}^{+}\right]$467.1941, found 467.1937 .

Data for (2S,3S,4R,5S)-3,4-bis(benzyloxy)-5-(benzyloxymethyl)-2-cyano-1-hydroxypyrrolidine (C-2) (Ref. [60]): Colourless syrup, $1.32 \mathrm{~g}, 96 \%$ yield from nitrone $\mathrm{C}(1.30 \mathrm{~g}, 3.12 \mathrm{mmol}) ;[\alpha]_{\mathrm{D}}{ }^{23}+8.1(c 1.5 \mathrm{in}$ $\left.\mathrm{CH}_{2} \mathrm{Cl}_{2}\right) ; v_{\max } / \mathrm{cm}^{-1}: 3306(\mathrm{~m}), 3030(\mathrm{~m}), 2925(\mathrm{~s}), 2855(\mathrm{~s}), 2251(\mathrm{w}), 1497(\mathrm{~m}), 1454(\mathrm{~s}), 1362(\mathrm{~m}), 1209$ (m), 1144 (s), 1102 (s), 1027 (s), 736 (s), 697 (vs); ${ }^{1} \mathrm{H}$ NMR (500 MHz, $\left.\mathrm{CDCl}_{3}\right) \delta$ (ppm.): 7.34-7.23 (m, $15 \mathrm{H}), 6.72(\mathrm{~s}, 1 \mathrm{H}, \mathrm{OH}), 4.69-4.44(\mathrm{~m}, 6 \mathrm{H}), 4.33(\mathrm{t}, J=5.5 \mathrm{~Hz}, 1 \mathrm{H}, \mathrm{H}-3), 4.28(\mathrm{~d}, J=5.6 \mathrm{~Hz}, 1 \mathrm{H} . \mathrm{H}-2), 4.22$ $(\mathrm{t}, J=5.7 \mathrm{~Hz}, 1 \mathrm{H}, \mathrm{H}-4), 3.72(\mathrm{dd}, J=9.6 \mathrm{~Hz}$ and $6.9 \mathrm{~Hz}, 1 \mathrm{H}, \mathrm{H}-6), 3.66(\mathrm{dd}, J=9.3 \mathrm{~Hz}$ and $6.7 \mathrm{~Hz}, 1 \mathrm{H}$, H-6'), 3.55-3.51 (m, 1H, H-5); ${ }^{13} \mathrm{C}$ NMR (125 MHz, $\left.\mathrm{CDCl}_{3}\right) \delta$ (ppm): 137.79, 137.70, 136.7, 128.6, 128.45, 128.43, 128.2, 128.0, 127.9, 127.89, 127.81, 116.4, 80.2 (C-3), 75.8 (C-4), $73.8\left(\mathrm{PhCH}_{2}\right), 73.4\left(\mathrm{PhCH}_{2}\right), 73.1$ $\left(\mathrm{PhCH}_{2}\right), 68.9$ (C-5), 67.4 (C-6), 60.4 (C-2); HRMS (ESI): calcd for $\mathrm{C}_{27} \mathrm{H}_{28} \mathrm{O}_{4} \mathrm{~N}_{2} \mathrm{Na}^{+}\left[\mathrm{M}+\mathrm{Na}^{+}\right]$467.1941, found 467.1939 .

Data for (2R,3R,4S,5R)-3,4-bis(benzyloxy)-5-(benzyloxymethyl)-2-cyano-1-hydroxypyrrolidine (D-2) (Ref. [61]): Colourless syrup, $1.19 \mathrm{~g}$, 92\% yield from nitrone $\mathbf{D}(1.22 \mathrm{~g}, 2.93 \mathrm{mmol}) ;[\alpha]_{\mathrm{D}}{ }^{23}-10.2(\mathrm{c}$ 1.0 in $\left.\mathrm{CH}_{2} \mathrm{Cl}_{2}\right) ; v_{\max } / \mathrm{cm}^{-1}$ : $3328(\mathrm{~m}), 3030(\mathrm{~m}), 2925(\mathrm{~s}), 2870(\mathrm{~s}), 2251(\mathrm{w}), 1497(\mathrm{~m}), 1454(\mathrm{~s}), 1362(\mathrm{~m})$, 1209 (m), 1145 (s), 1102 (s), 1027 (s), 736 (s), 697 (vs); $\left.{ }^{1} \mathrm{H} \mathrm{NMR} \mathrm{(500} \mathrm{MHz,} \mathrm{CDCl}_{3}\right) \delta(\mathrm{ppm}):$ 7.36-7.22 (m, $15 \mathrm{H}), 6.78(\mathrm{~s}, 1 \mathrm{H}, \mathrm{OH}), 4.69-4.43(\mathrm{~m}, 6 \mathrm{H}), 4.33(\mathrm{t}, J=5.5 \mathrm{~Hz}, 1 \mathrm{H}, \mathrm{H}-3), 4.28(\mathrm{~d}, J=5.6 \mathrm{~Hz}, 1 \mathrm{H} . \mathrm{H}-2)$, $4.22(\mathrm{t}, J=5.7 \mathrm{~Hz}, 1 \mathrm{H}, \mathrm{H}-4), 3.72(\mathrm{dd}, J=9.6 \mathrm{~Hz}$ and $6.9 \mathrm{~Hz}, 1 \mathrm{H}, \mathrm{H}-6), 3.65(\mathrm{dd}, J=9.3 \mathrm{~Hz}$ and $6.7 \mathrm{~Hz}$, 1H, H-6'), 3.55-3.51 (m, 1H, H-5); ${ }^{13} \mathrm{C}$ NMR $\left(125 \mathrm{MHz}, \mathrm{CDCl}_{3}\right) \delta(\mathrm{ppm}): 137.79,137.71,136.7,128.6$, 128.46, 128.43, 128.2, 128.0, 127.89, 127.81, 116.5, 80.2 (C-3), $75.8(\mathrm{C}-4), 73.8\left(\mathrm{PhCH}_{2}\right), 73.4\left(\mathrm{PhCH}_{2}\right), 73.1$ $\left(\mathrm{PhCH}_{2}\right), 69.0$ (C-5), 67.4 (C-6), 60.4 (C-2); HRMS (ESI): calcd for $\mathrm{C}_{27} \mathrm{H}_{28} \mathrm{O}_{4} \mathrm{~N}_{2} \mathrm{Na}^{+}\left[\mathrm{M}+\mathrm{Na}^{+}\right]$467.1941, found 467.1938 .

(2R,3R,4R,5S)-3,4-bis(benzyloxy)-5-(benzyloxymethyl)-2-cyano-1-hydroxypyrrolidine (E-2a) and (2S,3R,4R,5S)-3,4-bis(benzyloxy)-5-(benzyloxymethyl)-2-cyano-1-hydroxypyrrolidine (E-2b): 92\% total yield from nitrone $\mathrm{E}(1.34 \mathrm{~g}, 3.21 \mathrm{mmol})$.

Data for E-2a: White solid, $821 \mathrm{mg}, 58 \%$ yield; m.p. $117-119^{\circ} \mathrm{C} ;[\alpha]_{\mathrm{D}}{ }^{23}+7.8\left(\mathrm{c} 0.7 \mathrm{in} \mathrm{CH}_{2} \mathrm{Cl}_{2}\right) ; v_{\max } / \mathrm{cm}^{-1}$ : $3308(\mathrm{~m}), 3028(\mathrm{~m}), 2870(\mathrm{~m}), 2245(\mathrm{w}), 1497(\mathrm{~m}), 1453(\mathrm{~m}), 1361(\mathrm{~m}), 1144(\mathrm{~m}), 1109(\mathrm{~s}), 1043(\mathrm{~m})$, $1028(\mathrm{~m}), 736(\mathrm{vs}), 693(\mathrm{vs}) ;{ }^{1} \mathrm{HNMR}\left(500 \mathrm{MHz}, \mathrm{CDCl}_{3}\right) \delta(\mathrm{ppm}): 7.37-7.23(\mathrm{~m}, 15 \mathrm{H}), 5.75(\mathrm{~s}, 1 \mathrm{H}, \mathrm{OH})$, 4.56-4.45 (m, 6H), $4.15(\mathrm{dd}, J=5.6$ and $1.4 \mathrm{~Hz}, 1 \mathrm{H}, \mathrm{H}-2), 4.00(\mathrm{dd}, J=6.1$ and $1.4 \mathrm{~Hz}, 1 \mathrm{H}, \mathrm{H}-4), 3.83$ $(\mathrm{t}, J=9.1 \mathrm{~Hz}, 1 \mathrm{H}), 3.73(\mathrm{~m}, 2 \mathrm{H}, \mathrm{H}-6), 3.39-3.35(\mathrm{~m}, 1 \mathrm{H}, \mathrm{H}-5) ;{ }^{13} \mathrm{C} \mathrm{NMR}\left(125 \mathrm{MHz}, \mathrm{CDCl}_{3}\right) \delta(\mathrm{ppm})$ : 137.8, 137.2, 136.4, 128.6, 128.5, 128.4, 128.3, 128.0, 127.91, 127.90, 127.83, 127.81, 118.35, 83.9 (C-3), 80.0 (C-4), $73.5\left(\mathrm{PhCH}_{2}\right), 72.4\left(\mathrm{PhCH}_{2}\right), 72.3\left(\mathrm{PhCH}_{2}\right), 69.1$ (C-6), 67.1 (C-5), 63.2 (C-2); HRMS (ESI): calcd for $\mathrm{C}_{27} \mathrm{H}_{28} \mathrm{O}_{4} \mathrm{~N}_{2} \mathrm{Na}^{+}\left[\mathrm{M}+\mathrm{Na}^{+}\right]$467.1941, found 467.1938.

Data for E-2b: White solid, $491 \mathrm{mg}, 35 \%$ yield; m.p. $111-114{ }^{\circ} \mathrm{C} ;[\alpha]_{\mathrm{D}}{ }^{23}-38.1$ (c 1.0 in $\left.\mathrm{CH}_{2} \mathrm{Cl}_{2}\right)$; $v_{\max } / \mathrm{cm}^{-1}: 3359(\mathrm{~m}), 3031(\mathrm{~m}), 2868(\mathrm{~m}), 2237(\mathrm{w}), 1496(\mathrm{~m}), 1454(\mathrm{~m}), 1362(\mathrm{~m}), 1207(\mathrm{~m}), 1097(\mathrm{~s})$, 1027 (m), 737 (vs), 697 (vs); ${ }^{1} \mathrm{H}$ NMR (500 MHz, $\left.\mathrm{CDCl}_{3}\right) \delta(\mathrm{ppm}): 7.35-7.21(\mathrm{~m}, 15 \mathrm{H}), 5.98(\mathrm{~s}, 1 \mathrm{H}, \mathrm{OH})$, $4.59-4.48(\mathrm{~m}, 6 \mathrm{H}), 4.37(\mathrm{~d}, J=6.4 \mathrm{~Hz}, 1 \mathrm{H}, \mathrm{H}-2), 4.18(\mathrm{dd}, J=7.7$ and $4.3 \mathrm{~Hz}, 1 \mathrm{H}, \mathrm{H}-4), 4.07(\mathrm{dd}, J=6.3$ and $4.4 \mathrm{~Hz}, 1 \mathrm{H}, \mathrm{H}-3), 3.80(\mathrm{dd}, J=9.7$ and $5.7 \mathrm{~Hz}, 1 \mathrm{H}, \mathrm{H}-6), 3.68\left(\mathrm{dd}, J=9.6\right.$ and $\left.5.7 \mathrm{~Hz}, 1 \mathrm{H}, \mathrm{H}-6^{\prime}\right)$, 3.63-3.59 (m, 1H, H-5); ${ }^{13} \mathrm{C} \mathrm{NMR}\left(125 \mathrm{MHz}, \mathrm{CDCl}_{3}\right) \delta$ (ppm): 137.7, 137.5, 136.6, 128.6, 128.4, 128.3, $128.2,127.9,127.8,127.78,127.73,114.6,81.1(\mathrm{C}-3), 80.8(\mathrm{C}-4), 73.5\left(\mathrm{PhCH}_{2}\right), 73.0\left(\mathrm{PhCH}_{2}\right), 72.9\left(\mathrm{PhCH}_{2}\right)$, 67.4 (C-6), 66.7 (C-5), 60.6 (C-2); HRMS (ESI): calcd for $\mathrm{C}_{27} \mathrm{H}_{28} \mathrm{O}_{4} \mathrm{~N}_{2} \mathrm{Na}^{+}\left[\mathrm{M}+\mathrm{Na}^{+}\right]$467.1941, found 467.1939.

(2S,3S,4S,5R)-3,4-bis(benzyloxy)-5-(benzyloxymethyl)-2-cyano-1-hydroxypyrrolidine (F-2a) and (2R,3S,4S,5R)-3,4-bis(benzyloxy)-5-(benzyloxymethyl)-2-cyano-1-hydroxypyrrolidine (F-2b): $96 \%$ total yield from nitrone $\mathrm{F}(1.50 \mathrm{~g}, 3.60 \mathrm{mmol})$. 
Data for F-2a: White solid, $985 \mathrm{mg}, 59 \%$ yield; m.p. $118-119{ }^{\circ} \mathrm{C} ;[\alpha]_{\mathrm{D}}{ }^{23}-5.5\left(\mathrm{c} 1.0\right.$ in $\left.\mathrm{CH}_{2} \mathrm{Cl}_{2}\right) ; v_{\mathrm{max}} / \mathrm{cm}^{-1}$ : $3326(\mathrm{~m}), 3028(\mathrm{~m}), 2911(\mathrm{~m}), 2870(\mathrm{~m}), 2245(\mathrm{w}), 1497(\mathrm{~m}), 1454(\mathrm{~s}), 1241(\mathrm{~m}), 1216(\mathrm{~m}), 1144(\mathrm{~s}), 1110$ (vs), 736 (vs), 693 (vs); ${ }^{1} \mathrm{H}$ NMR (500 MHz, $\left.\mathrm{CDCl}_{3}\right) \delta(\mathrm{ppm}): 7.37-7.23$ (m,15H), 5.70 (s, 1H, OH), 4.56-4.45 $(\mathrm{m}, 6 \mathrm{H}), 4.15(\mathrm{dd}, J=5.7$ and $1.4 \mathrm{~Hz}, 1 \mathrm{H}, \mathrm{H}-2), 4.00(\mathrm{dd}, J=6.1$ and $1.4 \mathrm{~Hz}, 1 \mathrm{H}, \mathrm{H}-4), 3.83(\mathrm{dd}, J=9.2$ and $7.7 \mathrm{~Hz}, 1 \mathrm{H}, \mathrm{H}-3), 3.75-3.71(\mathrm{~m}, 2 \mathrm{H}, \mathrm{H}-6), 3.39-3.35(\mathrm{~m}, 1 \mathrm{H}) ;{ }^{13} \mathrm{C} \mathrm{NMR}\left(125 \mathrm{MHz}, \mathrm{CDCl}_{3}\right) \delta(\mathrm{ppm})$ : 137.8, 137.2, 136.4, 128.6, 128.5, 128.4, 128.3, 128.0, 127.91, 127.90, 127.83, 127.81, 118.3, 83.9 (C-3), 80.0 (C-4), $73.5\left(\mathrm{PhCH}_{2}\right), 72.4\left(\mathrm{PhCH}_{2}\right), 72.3\left(\mathrm{PhCH}_{2}\right), 69.1$ (C-6), 67.1 (C-5), 63.2 (C-2); HRMS (ESI): calcd for $\mathrm{C}_{27} \mathrm{H}_{28} \mathrm{O}_{4} \mathrm{~N}_{2} \mathrm{Na}^{+}\left[\mathrm{M}+\mathrm{Na}^{+}\right]$467.1941, found 467.1938.

Data for F-2b: White solid, $612 \mathrm{mg}, 37 \%$ yield; m.p. $113-115{ }^{\circ} \mathrm{C}$; $[\alpha]_{\mathrm{D}}{ }^{23}+35.3\left(\mathrm{c} 1.0\right.$ in $\left.\mathrm{CH}_{2} \mathrm{Cl}_{2}\right)$; $v_{\max } / \mathrm{cm}^{-1}$ : $3359(\mathrm{~m}), 3031(\mathrm{~m}), 2868(\mathrm{~m}), 2239(\mathrm{w}), 1496(\mathrm{~m}), 1454(\mathrm{~m}), 1362(\mathrm{~m}), 1207(\mathrm{~s}), 1097(\mathrm{~s}), 1027$ (m), 737 (vs), 697 (vs); ${ }^{1} \mathrm{H}$ NMR (500 MHz, $\left.\mathrm{CDCl}_{3}\right) \delta$ (ppm):7.35-7.21 (m, 15H), 6.04 (s, 1H, OH), 4.59 $-4.47(\mathrm{~m}, 6 \mathrm{H}), 4.37(\mathrm{~d}, J=6.3 \mathrm{~Hz}, 1 \mathrm{H}, \mathrm{H}-2), 4.18(\mathrm{dd}, J=7.7$ and $4.3 \mathrm{~Hz}, 1 \mathrm{H}, \mathrm{H}-4), 4.07(\mathrm{dd}, J=6.3$ and $4.4 \mathrm{~Hz}, 1 \mathrm{H}, \mathrm{H}-3$ ), $3.80(\mathrm{dd}, J=9.7$ and $5.7 \mathrm{~Hz}, 1 \mathrm{H}, \mathrm{H}-6), 3.68\left(\mathrm{dd}, J=9.6\right.$ and $\left.5.7 \mathrm{~Hz}, 1 \mathrm{H}, \mathrm{H}-6^{\prime}\right), 3.63-3.59$ (m, 1H, H-5); $\left.{ }^{13} \mathrm{C} \mathrm{NMR} \mathrm{(125} \mathrm{MHz,} \mathrm{CDCl}_{3}\right) \delta$ (ppm): 137.7, 137.5, 136.6, 128.6, 128.4, 128.3, 128.2, 127.9, 127.8, 127.78, 127.74, 114.6, $81.1(\mathrm{C}-3), 80.8(\mathrm{C}-4), 73.5\left(\mathrm{PhCH}_{2}\right), 73.0\left(\mathrm{PhCH}_{2}\right), 72.9\left(\mathrm{PhCH}_{2}\right), 67.4(\mathrm{C}-6)$, 66.7 (C-5), 60.6 (C-2); HRMS (ESI): calcd for $\mathrm{C}_{27} \mathrm{H}_{28} \mathrm{O}_{4} \mathrm{~N}_{2} \mathrm{Na}^{+}\left[\mathrm{M}+\mathrm{Na}^{+}\right]$467.1941, found 467.1939.

Data for $(2 R, 3 R, 4 S, 5 S)-3,4$-bis(benzyloxy)-5-(benzyloxymethyl)-2-cyano-1-hydroxypyrrolidine (G-2) (Ref. [61]): White solid, $1.39 \mathrm{~g}, 87 \%$ yield from nitrone G (1.51 g, $3.62 \mathrm{mmol}) ;$ m.p. $106-109{ }^{\circ} \mathrm{C}$; $[\alpha]_{\mathrm{D}}^{23}+0.6\left(c 1.0\right.$ in $\left.\mathrm{CH}_{2} \mathrm{Cl}_{2}\right) ; v_{\max } / \mathrm{cm}^{-1}: 3403(\mathrm{~m}), 3029(\mathrm{~m}), 2922(\mathrm{~m}), 2871(\mathrm{~m}), 2242(\mathrm{w}), 1497(\mathrm{~m})$, 1453 (m), 1352 (m), 1224 (m), 1145 (s), 1101 (s), 1027 (s), 742 (s), 694 (vs); ${ }^{1} \mathrm{HNMR}\left(500 \mathrm{MHz}, \mathrm{CDCl}_{3}\right)$ $\delta$ (ppm):7.35-7.24 (m,15H), $6.05(\mathrm{~s}, 1 \mathrm{H}, \mathrm{OH}), 4.70-4.43(\mathrm{~m}, 6 \mathrm{H}), 4.10-4.05(\mathrm{~m}, 2 \mathrm{H}, \mathrm{H}-3, \mathrm{H}-2), 3.83(\mathrm{t}$, $J=6.1 \mathrm{~Hz}, 1 \mathrm{H}, \mathrm{H}-4), 3.53-3.51$ (m, 2H, H-6, H-6'), 3.31-3.30 (m,1H, H-5); ${ }^{13} \mathrm{C}$ NMR $\left(125 \mathrm{MHz}, \mathrm{CDCl}_{3}\right)$ $\delta$ (ppm): 137.6, 137.4, 136.7, 128.6, 128.48, 128.44, 128.2, 128.13, 128.11, 127.98, 127.90, 127.88, 127.86, 118.5, 77.3 (C-3), $75.2(\mathrm{C}-4), 73.3\left(\left(\mathrm{PhCH}_{2}\right)\right), 72.8\left(\mathrm{PhCH}_{2}\right), 72.2\left(\mathrm{PhCH}_{2}\right), 72.0$ (C-5), 68.0 (C-6), 61.8 (C-2); HRMS (ESI): calcd for $\mathrm{C}_{27} \mathrm{H}_{28} \mathrm{O}_{4} \mathrm{~N}_{2} \mathrm{Na}^{+}\left[\mathrm{M}+\mathrm{Na}^{+}\right]$467.1941, found 467.1936.

Data for (2S,3S,4R,5R)-3,4-bis(benzyloxy)-5-(benzyloxymethyl)-2-cyano-1-hydroxypyrrolidine (H-2): White solid, $1.45 \mathrm{~g}, 91 \%$ yield from nitrone $\mathbf{H}(1.50 \mathrm{~g}, 3.60 \mathrm{mmol}) ; \mathrm{m} . \mathrm{p} .109-111^{\circ} \mathrm{C} ;[\alpha]_{\mathrm{D}}{ }^{23}-1.4$ (c 1.1 in $\left.\mathrm{CH}_{2} \mathrm{Cl}_{2}\right)$; $v_{\max } / \mathrm{cm}^{-1}: 3407(\mathrm{~m}), 3029(\mathrm{~m}), 2873(\mathrm{~m}), 2242(\mathrm{w}), 1497(\mathrm{~m}), 1453(\mathrm{~m}), 1352(\mathrm{~m})$, 1225 (m), 1149 (s), 1102 (s), 1035 (s), 1028 (s), 743 (vs), 694 (vs); $\left.{ }^{1} \mathrm{H} \mathrm{NMR} \mathrm{(500} \mathrm{MHz,} \mathrm{CDCl}{ }_{3}\right) \delta$ (ppm): 7.34-7.23 (m,15H), $6.11(\mathrm{~s}, 1 \mathrm{H}, \mathrm{OH}), 4.68-4.43(\mathrm{~m}, 6 \mathrm{H}), 4.10-4.05(\mathrm{~m}, 2 \mathrm{H}, \mathrm{H}-3, \mathrm{H}-2), 3.81(\mathrm{t}, J=5.1 \mathrm{~Hz}$, $1 \mathrm{H}, \mathrm{H}-4), 3.53-3.48$ (m, 2H, H-6, H-6'), 3.31-3.28 (m,1H, H-5); ${ }^{13} \mathrm{C} \mathrm{NMR}\left(125 \mathrm{MHz}, \mathrm{CDCl}_{3}\right) \delta(\mathrm{ppm})$ : 137.6, 137.4, 136.7, 128.6, 128.49, 128.45, 128.2, 128.13, 128.11, 127.98, 127.91, 127.8, 118.6, 77.3 (C-3), 75.2 (C-4), $73.3\left(\mathrm{PhCH}_{2}\right), 72.8\left(\mathrm{PhCH}_{2}\right), 72.2\left(\mathrm{PhCH}_{2}\right), 72.0$ (C-5), 68.0 (C-6), 61.8 (C-2); HRMS (ESI): calcd for $\mathrm{C}_{27} \mathrm{H}_{28} \mathrm{O}_{4} \mathrm{~N}_{2} \mathrm{Na}^{+}\left[\mathrm{M}+\mathrm{Na}^{+}\right] 467.1941$, found 467.1933.

3.3.2. General Procedure for Synthesis of Hydroxylamines A-3, B-3, C-3, D-3, E-3a, E-3b, F-3a, F-3b, G-3 and H-3 with A-3 as an Example

Hydroxylamine A-2 (1.28 g, $2.88 \mathrm{mmol})$ was dissolved in methanol $(10 \mathrm{~mL})$, followed by Boc ${ }_{2} \mathrm{O}$ $(1.46 \mathrm{~mL}, 6.30 \mathrm{mmol})$ and Raney $\mathrm{Ni}(1.0 \mathrm{~g}, 60 \%)$. The suspension was stirred under hydrogen atmosphere for $4 \mathrm{~h}$ when TLC showed completion of the reaction. Hydrogen was replaced by nitrogen, and catalyst was removed from the reaction mixture by filtration. The filtrate was concentrated in vacuo to afford the crude product as a colourless oil. The intermediate was dissolved in dichloromethane $(10 \mathrm{~mL})$ and cooled to $0{ }^{\circ} \mathrm{C}$, trifluoroacetic acid $(0.54 \mathrm{~mL}, 7.20 \mathrm{mmol})$ was added dropwise. After stirring at room temperature for $3 \mathrm{~h}$, TLC showed completion of the reaction. The mixture was neutralized by aqueous $\mathrm{NaHCO}_{3}$ and extracted with dichloromethane $(3 \times 10 \mathrm{~mL})$. The organic phases were combined, washed with brine and dried over $\mathrm{MgSO}_{4} . \mathrm{After}$ concentrated in vacuo, the crude product was purified by flash column chromatography (silica gel, dichloromethane/methanol = 25:1) to give diamine A-3 (colourless syrup, $1.05 \mathrm{~g}$, 85\% yield for two steps). Data for (2S,3S,4S,5S)-2-(aminomethyl)-3,4-bis(benzyloxy)-5-(benzyloxymethyl)pyrrolidine 
(A-3): $[\alpha]_{\mathrm{D}}{ }^{23}-16.3$ (c 1.0 in $\left.\mathrm{CH}_{2} \mathrm{Cl}_{2}\right) ; v_{\max } / \mathrm{cm}^{-1}: 3031$ (m), 2923 (s), 2865 (s), 1682 (vs), 1453 (m), 1203 (vs), 1130 (vs), 1027 (m), 737 (s), 721 (s), 697 (s); ${ }^{1} \mathrm{H} \mathrm{NMR}\left(500 \mathrm{MHz}, \mathrm{CDCl}_{3}\right) \delta(\mathrm{ppm}): 7.34-7.24(\mathrm{~m}, 15 \mathrm{H})$, $4.55-4.49(\mathrm{~m}, 6 \mathrm{H}), 3.91(\mathrm{t}, J=3.8 \mathrm{~Hz}, 1 \mathrm{H}), 3.76(\mathrm{t}, J=3.8 \mathrm{~Hz}, 1 \mathrm{H}), 3.56-3.50(\mathrm{~m}, 2 \mathrm{H}), 3.32(\mathrm{q}, J=5.0 \mathrm{~Hz}$, $1 \mathrm{H}), 3.15-3.12(\mathrm{~m}, 1 \mathrm{H}), 2.79-2.71(\mathrm{~m}, 2 \mathrm{H}), 1.77\left(\mathrm{~s}, 3 \mathrm{H}, \mathrm{NH}, \mathrm{NH}_{2}\right) ;{ }^{13} \mathrm{C} \mathrm{NMR}\left(125 \mathrm{MHz}, \mathrm{CDCl}_{3}\right) \delta(\mathrm{ppm})$ : 138.2, 138.16, 138.15, 128.44, 128.41, 127.8, 127.78, 127.75, 127.71, 127.6, 87.3, 86.1, 73.2, 71.9, 71.7, 70.3, 64.1, 61.6, 44.3; HRMS (ESI): calcd for $\mathrm{C}_{27} \mathrm{H}_{33} \mathrm{O}_{3} \mathrm{~N}_{2}{ }^{+}\left[\mathrm{M}+\mathrm{H}^{+}\right]$433.2486, found 433.2478.

Data for (2R,3R,4R,5R)-2-(aminomethyl)-3,4-bis(benzyloxy)-5-(benzyloxymethyl)pyrrolidine (B-3): Colourless syrup, $380 \mathrm{mg}$, 88\% yield from hydroxylamine B-2 $(450 \mathrm{mg}, 1.01 \mathrm{mmol}) ;[\alpha]_{\mathrm{D}}{ }^{23}+11.8(c 0.8$ in $\left.\mathrm{CH}_{2} \mathrm{Cl}_{2}\right) ; v_{\max } / \mathrm{cm}^{-1}: 3030(\mathrm{~m}), 2866(\mathrm{~m}), 1682(\mathrm{vs}), 1496(\mathrm{~m}), 1453(\mathrm{~m}), 1204(\mathrm{vs}), 1130$ (vs), $1027(\mathrm{~m})$, $736(\mathrm{~s}), 720(\mathrm{~s}), 697(\mathrm{~s}) ;{ }^{1} \mathrm{H}$ NMR $\left(500 \mathrm{MHz}, \mathrm{CDCl}_{3}\right) \delta(\mathrm{ppm}): 7.34-7.24(\mathrm{~m}, 15 \mathrm{H}), 4.55-4.49(\mathrm{~m}, 6 \mathrm{H}), 3.91$ $(\mathrm{t}, J=3.8 \mathrm{~Hz}, 1 \mathrm{H}), 3.76(\mathrm{t}, J=3.8 \mathrm{~Hz}, 1 \mathrm{H}), 3.56-3.50(\mathrm{~m}, 2 \mathrm{H}), 3.32(\mathrm{q}, J=5.0 \mathrm{~Hz}, 1 \mathrm{H}), 3.16-3.12(\mathrm{~m}, 1 \mathrm{H})$, 2.79-2.71 (m, 2H), $1.76\left(\mathrm{~s}, 3 \mathrm{H}, \mathrm{NH}, \mathrm{NH}_{2}\right) ;{ }^{13} \mathrm{C} \mathrm{NMR}\left(125 \mathrm{MHz}, \mathrm{CDCl}_{3}\right) \delta(\mathrm{ppm}): 138.2,138.16,138.15$, $128.44,128.41,127.8,127.78,127.75,127.71,127.6,87.3,86.1,73.2,71.9,71.7,70.3,64.1,61.6,44.3$; HRMS (ESI): calcd for $\mathrm{C}_{27} \mathrm{H}_{33} \mathrm{O}_{3} \mathrm{~N}_{2}{ }^{+}\left[\mathrm{M}+\mathrm{H}^{+}\right]$433.2486, found 433.2478.

Data for (2S,3S,4R,5S)-2-(aminomethyl)-3,4-bis(benzyloxy)-5-(benzyloxymethyl)pyrrolidine (C-3): Colourless syrup, $372 \mathrm{mg}$, 86\% yield from hydroxylamine C-2 $(450 \mathrm{mg}, 1.01 \mathrm{mmol}) ;[\alpha]_{\mathrm{D}}{ }^{22}+13.3$ (c 1.0 in $\left.\mathrm{CH}_{2} \mathrm{Cl}_{2}\right) ; v_{\max } / \mathrm{cm}^{-1}: 3328(\mathrm{~m}), 3030(\mathrm{~m}), 2864(\mathrm{~m}), 1495(\mathrm{~m}), 1453(\mathrm{~s}), 1143(\mathrm{~s}), 1094(\mathrm{~s}), 1027(\mathrm{~m})$, 736 (s), 696 (vs); ${ }^{1} \mathrm{H}$ NMR (500 MHz, CDCl $) \delta(\mathrm{ppm}): 7.32-7.25(\mathrm{~m}, 15 \mathrm{H}), 4.73-4.43(\mathrm{~m}, 6 \mathrm{H}), 4.04(\mathrm{t}$, $J=4.1 \mathrm{~Hz}, 1 \mathrm{H}), 3.70-3.61(\mathrm{~m}, 3 \mathrm{H}), 3.42(\mathrm{q}, J=6.6 \mathrm{~Hz}, 1 \mathrm{H}), 3.30(\mathrm{q}, J=6.3 \mathrm{~Hz}, 1 \mathrm{H}), 2.77(\mathrm{dd}, J=12.6$ and $4.2 \mathrm{~Hz}, 1 \mathrm{H}), 2.59(\mathrm{dd}, J=12.6$ and $6.5 \mathrm{~Hz}, 1 \mathrm{H}), 1.50\left(\mathrm{~s}, 3 \mathrm{H}, \mathrm{NH}, \mathrm{NH}_{2}\right) ;{ }^{13} \mathrm{C} \mathrm{NMR}\left(125 \mathrm{MHz}, \mathrm{CDCl}_{3}\right) \delta$ (ppm): 138.6, 138.2, 138.1, 128.44, 128.41, 128.3, 127.8, 127.78, 127.76, 127.6, 127.5, 81.9, 78.0, 73.3, 73.2, 72.3, 69.7, 61.9, 59.0, 45.2; HRMS (ESI): calcd for $\mathrm{C}_{27} \mathrm{H}_{33} \mathrm{O}_{3} \mathrm{~N}_{2}{ }^{+}\left[\mathrm{M}+\mathrm{H}^{+}\right]$433.2486, found 433.2479.

Data for (2R,3R,4S,5R)-2-(aminomethyl)-3,4-bis(benzyloxy)-5-(benzyloxymethyl)pyrrolidine (D-3): Colourless syrup, $372 \mathrm{mg}$, 86\% yield from hydroxylamine D-2 (450 mg, $1.01 \mathrm{mmol}) ;[\alpha]_{\mathrm{D}}{ }^{23}-19.6$ (c 1.2 in $\left.\mathrm{CH}_{2} \mathrm{Cl}_{2}\right) ; v_{\max } / \mathrm{cm}^{-1}: 3292(\mathrm{w}), 3029(\mathrm{~m}), 2859(\mathrm{~m}), 1585(\mathrm{~m}), 1495(\mathrm{~m}), 1453(\mathrm{~s}), 1143(\mathrm{~s}), 1094(\mathrm{~s}), 1027$ (m), $734(\mathrm{~s}), 696(\mathrm{vs}) ;{ }^{1} \mathrm{H}$ NMR $\left(500 \mathrm{MHz}, \mathrm{CDCl}_{3}\right) \delta(\mathrm{ppm}): 7.32-7.25(\mathrm{~m}, 15 \mathrm{H}), 4.73-4.43(\mathrm{~m}, 6 \mathrm{H}), 4.04$ $(\mathrm{t}, J=4.1 \mathrm{~Hz}, 1 \mathrm{H}), 3.70-3.61(\mathrm{~m}, 3 \mathrm{H}), 3.42(\mathrm{q}, J=6.6 \mathrm{~Hz}, 1 \mathrm{H}), 3.30(\mathrm{q}, J=6.3 \mathrm{~Hz}, 1 \mathrm{H}), 2.77(\mathrm{dd}, J=12.6$ and $4.2 \mathrm{~Hz}, 1 \mathrm{H}), 2.59(\mathrm{dd}, J=12.6$ and $4.3 \mathrm{~Hz}, 1 \mathrm{H}), 1.50\left(\mathrm{~s}, 3 \mathrm{H}, \mathrm{NH}, \mathrm{NH}_{2}\right) ;{ }^{13} \mathrm{C} \mathrm{NMR}\left(125 \mathrm{MHz}, \mathrm{CDCl}_{3}\right)$ $\delta$ (ppm): 138.6, 138.2, 138.1, 128.44, 128.41, 128.3, 127.8, 127.78, 127.76, 127.6, 127.5, 81.9, 78.0, 73.3, 73.2, 72.3, 69.7, 61.9, 59.0, 45.2; HRMS (ESI): calcd for $\mathrm{C}_{27} \mathrm{H}_{33} \mathrm{O}_{3} \mathrm{~N}_{2}{ }^{+}\left[\mathrm{M}+\mathrm{H}^{+}\right]$433.2486, found 433.2478 .

Data for (2R,3R,4R,5S)-2-(aminomethyl)-3,4-bis(benzyloxy)-5-(benzyloxymethyl)pyrrolidine (E-3a): Colourless syrup, $379 \mathrm{mg}$, 88\% yield from hydroxylamine E-2a (450 mg, $1.01 \mathrm{mmol}) ;[\alpha]_{\mathrm{D}}{ }^{23}+12.1$ (c 1.0 in $\left.\mathrm{CH}_{2} \mathrm{Cl}_{2}\right) ; v_{\max } / \mathrm{cm}^{-1}: 3062(\mathrm{w}), 3029(\mathrm{~m}), 2923(\mathrm{~s}), 2860(\mathrm{~s}), 1688(\mathrm{~m}), 1496(\mathrm{~m}), 1453(\mathrm{~s}), 1201(\mathrm{~m}), 1093$ (vs), 735 (vs), 697 (vs); $\left.{ }^{1} \mathrm{H} \mathrm{NMR} \mathrm{(500} \mathrm{MHz,} \mathrm{CDCl}_{3}\right) \delta$ (ppm): 7.35-7.24 (m, 15H), 4.57-4.44 (m, 6H), 3.92 $(\mathrm{dd}, J=4.6$ and $1.1 \mathrm{~Hz}, 1 \mathrm{H}), 3.73(\mathrm{dd}, J=9.2$ and $6.0 \mathrm{~Hz}, 1 \mathrm{H}), 3.65(\mathrm{dd}, J=4.5$ and $1.1 \mathrm{~Hz}, 1 \mathrm{H}), 3.62(\mathrm{dd}$, $J=9.2$ and $6.8 \mathrm{~Hz}, 1 \mathrm{H}), 3.52(\mathrm{q}, J=5.95 \mathrm{~Hz}, 1 \mathrm{H}), 3.12-3.08(\mathrm{~m}, 1 \mathrm{H}), 2.85(\mathrm{dd}, 12.6$ and $4.9 \mathrm{~Hz}, 1 \mathrm{H}), 2.76$ $(\mathrm{dd}, J=12.6$ and $7.1 \mathrm{~Hz}, 1 \mathrm{H}), 1.61\left(\mathrm{~s}, 3 \mathrm{H}, \mathrm{NH}, \mathrm{NH}_{2}\right) ;{ }^{13} \mathrm{C} \mathrm{NMR}\left(125 \mathrm{MHz}, \mathrm{CDCl}_{3}\right) \delta(\mathrm{ppm}): 138.3,138.2$, 138.0, 128.48, 128.41, 128.3, 127.8, 127.79, 127.75, 127.72, 127.69, 127.63, 127.60, 85.8, 82.9, 73.4, 71.67, 71.64, 69.7, 66.0, 60.6, 45.5; HRMS (ESI): calcd for $\mathrm{C}_{27} \mathrm{H}_{33} \mathrm{O}_{3} \mathrm{~N}_{2}{ }^{+}\left[\mathrm{M}+\mathrm{H}^{+}\right]$433.2486, found 433.2477.

Data for (2S,3R,4R,5S)-2-(aminomethyl)-3,4-bis(benzyloxy)-5-(benzyloxymethyl)pyrrolidine (E-3b): Colourless syrup, $387 \mathrm{mg}$, 90\% yield from hydroxylamine E-2b $(450 \mathrm{mg}, 1.01 \mathrm{mmol}) ;[\alpha]_{\mathrm{D}}{ }^{23}-27.1(c$ 0.9 in $\left.\mathrm{CH}_{2} \mathrm{Cl}_{2}\right) ; v_{\max } / \mathrm{cm}^{-1}$ : $3062(\mathrm{w}), 3029(\mathrm{~m}), 2920(\mathrm{~s}), 2861$ (s), $1495(\mathrm{~m}), 1453(\mathrm{~s}), 1362(\mathrm{~m}), 1094(\mathrm{vs})$, 1027 (m), 735 (vs), 697 (vs); ${ }^{1} \mathrm{H} \mathrm{NMR}\left(500 \mathrm{MHz}, \mathrm{CDCl}_{3}\right) \delta$ (ppm): 7.34-7.25 (m, 15H), 4.57-4.38 (m, 6H), $4.04(\mathrm{dd}, J=4.6$ and $2.3 \mathrm{~Hz}, 1 \mathrm{H}), 3.96(\mathrm{dd}, J=5.3$ and $2.3 \mathrm{~Hz}, 1 \mathrm{H}), 3.67-3.60(\mathrm{~m}, 2 \mathrm{H}), 3.55(\mathrm{dd}, J=8.6$ and $6.3 \mathrm{~Hz}, 1 \mathrm{H}), 3.36(\mathrm{q}, J=6.1 \mathrm{~Hz}, 1 \mathrm{H}), 2.86-2.78(\mathrm{~m}, 2 \mathrm{H}), 1.63\left(\mathrm{~s}, 3 \mathrm{H}, \mathrm{NH}, \mathrm{NH}_{2}\right) ;{ }^{13} \mathrm{C} \mathrm{NMR}(125 \mathrm{MHz}$, $\left.\mathrm{CDCl}_{3}\right) \delta$ (ppm): 138.3, 138.2, 138.1, 128.4, 128.39, 128.35, 127.78, 127.74, 127.71, 127.6, 127.57, 127.56, 82.9, 82.5, 73.3, 72.2, 71.9, 69.5, 61.1, 58.5, 42.1; HRMS (ESI): calcd for $\mathrm{C}_{27} \mathrm{H}_{33} \mathrm{O}_{3} \mathrm{~N}_{2}{ }^{+}\left[\mathrm{M}+\mathrm{H}^{+}\right]$433.2486, found 433.2479 . 
Data for (2S,3S,4S,5R)-2-(aminomethyl)-3,4-bis(benzyloxy)-5-(benzyloxymethyl)pyrrolidine (F-3a): Colourless syrup, $359 \mathrm{mg}$, 83\% yield from hydroxylamine F-2a $(450 \mathrm{mg}, 1.01 \mathrm{mmol}) ;[\alpha]_{\mathrm{D}}{ }^{23}-9.3(c 1.0$ in $\left.\mathrm{CH}_{2} \mathrm{Cl}_{2}\right) ; v_{\max } / \mathrm{cm}^{-1}: 3087(\mathrm{w}), 3029(\mathrm{~m}), 2922(\mathrm{~s}), 2859(\mathrm{~s}), 1688(\mathrm{~m}), 1496(\mathrm{~m}), 1453(\mathrm{~s}), 1363(\mathrm{~m})$, 1201 (s), 1094 (vs), 736 (vs), 697 (vs); ${ }^{1} \mathrm{H}$ NMR $\left(500 \mathrm{MHz}, \mathrm{CDCl}_{3}\right) \delta$ (ppm):7.35-7.24 (m, 15H), 4.58-4.44 $(\mathrm{m}, 6 \mathrm{H}), 3.92(\mathrm{dd}, J=4.6$ and $1.2 \mathrm{~Hz}, 1 \mathrm{H}), 3.73(\mathrm{dd}, J=9.2$ and $6.0 \mathrm{~Hz}, 1 \mathrm{H}), 3.65(\mathrm{dd}, J=4.1$ and $1.2 \mathrm{~Hz}$,$) ,$ $3.62(\mathrm{dd}, J=9.2$ and $6.8 \mathrm{~Hz}, 1 \mathrm{H}), 3.54-3.50(\mathrm{~m}, 1 \mathrm{H}), 3.12-3.08(\mathrm{~m}, 1 \mathrm{H}), 2.84(\mathrm{dd}, J=12.6$ and $4.9 \mathrm{~Hz}, 1 \mathrm{H})$, $2.76(\mathrm{dd}, J=12.6$ and $7.1 \mathrm{~Hz}, 1 \mathrm{H}), 1.60\left(\mathrm{~s}, 3 \mathrm{H}, \mathrm{NH}, \mathrm{NH}_{2}\right) ;{ }^{13} \mathrm{C} \mathrm{NMR}\left(125 \mathrm{MHz}, \mathrm{CDCl}_{3}\right) \delta(\mathrm{ppm}): 138.3$, 138.2, 138.0, 128.48, 128.41, 128.3, 127.8, 127.79, 127.72, 127.69, 127.63, 127.60, 85.8, 82.9, 73.4, 71.69, 71.64, 69.7, 66.0, 60.6, 45.5; HRMS (ESI): calcd for $\mathrm{C}_{27} \mathrm{H}_{33} \mathrm{O}_{3} \mathrm{~N}_{2}{ }^{+}\left[\mathrm{M}+\mathrm{H}^{+}\right]$433.2486, found 433.2478.

Data for (2R,3S,4S,5R)-2-(aminomethyl)-3,4-bis(benzyloxy)-5-(benzyloxymethyl)pyrrolidine (F-3b): Colourless syrup, $367 \mathrm{mg}$, 85\% yield from hydroxylamine $\mathbf{F}-2 \mathbf{b}(450 \mathrm{mg}, 1.01 \mathrm{mmol}) ;[\alpha]_{\mathrm{D}}{ }^{22}+23.8($ c 1.0 in $\left.\mathrm{CH}_{2} \mathrm{Cl}_{2}\right) ; v_{\max } / \mathrm{cm}^{-1}: 3062(\mathrm{w}), 3029(\mathrm{~m}), 2922(\mathrm{~s}), 2858(\mathrm{~s}), 1495(\mathrm{~m}), 1453(\mathrm{~s}), 1362(\mathrm{~m}), 1093$ (vs), $1027(\mathrm{~m}), 734$ (s), $696(\mathrm{vs}) ;{ }^{1} \mathrm{H}$ NMR $\left(500 \mathrm{MHz}, \mathrm{CDCl}_{3}\right) \delta(\mathrm{ppm}): 7.34-7.25(\mathrm{~m}, 15 \mathrm{H}), 4.57-4.38(\mathrm{~m}, 6 \mathrm{H})$, $4.04(\mathrm{dd}, J=4.6$ and $2.3 \mathrm{~Hz}, 1 \mathrm{H}), 3.96(\mathrm{dd}, J=5.3$ and $2.3 \mathrm{~Hz}, 1 \mathrm{H}), 3.67-3.60(\mathrm{~m}, 2 \mathrm{H}), 3.55(\mathrm{dd}, J=8.6$ and $6.3 \mathrm{~Hz}, 1 \mathrm{H}), 3.36(\mathrm{q}, J=6.1 \mathrm{~Hz}, 1 \mathrm{H}), 2.86-2.78(\mathrm{~m}, 2 \mathrm{H}), 1.62\left(\mathrm{~s}, 3 \mathrm{H}, \mathrm{NH}, \mathrm{NH}_{2}\right) ;{ }^{13} \mathrm{C} \mathrm{NMR}(125 \mathrm{MHz}$, $\left.\mathrm{CDCl}_{3}\right) \delta$ (ppm): 138.3, 138.2, 138.1, 128.47, 128.42, 128.3, 127.8, 127.77, 127.74, 127.69, 127.60, 127.5, 82.9, 82.5, 73.4, 72.3, 71.9, 69.6, 61.1, 58.6, 42.1; HRMS (ESI): calcd for $\mathrm{C}_{27} \mathrm{H}_{33} \mathrm{O}_{3} \mathrm{~N}_{2}{ }^{+}\left[\mathrm{M}+\mathrm{H}^{+}\right]$433.2486, found 433.2478 .

Data for (2R,3R,4S,5S)-2-(aminomethyl)-3,4-bis(benzyloxy)-5-(benzyloxymethyl)pyrrolidine (G-3): Colourless syrup, $375 \mathrm{mg}$, 87\% yield from hydroxylamine G-2 (450 mg, $1.01 \mathrm{mmol}) ;[\alpha]_{\mathrm{D}}{ }^{22}+9.2$ (c 1.1 in $\left.\mathrm{CH}_{2} \mathrm{Cl}_{2}\right) ; v_{\max } / \mathrm{cm}^{-1}: 3362(\mathrm{w}), 3061(\mathrm{~m}), 3029(\mathrm{~m}), 2859(\mathrm{~s}), 1496(\mathrm{~m}), 1453(\mathrm{~s}), 1363(\mathrm{~m}), 1094(\mathrm{vs})$, 1027 (m), 735 (vs), 697 (vs); ${ }^{1} \mathrm{H}$ NMR (400 MHz, $\left.\mathrm{CDCl}_{3}\right) \delta(\mathrm{ppm}): 7.34-7.22(\mathrm{~m}, 15 \mathrm{H}), 4.60-4.42(\mathrm{~m}$, $6 \mathrm{H}), 3.75(\mathrm{t}, J=5.0 \mathrm{~Hz}, 1 \mathrm{H}), 3.55(\mathrm{t}, J=5.9 \mathrm{~Hz}, 1 \mathrm{H}), 3.49(\mathrm{q}, J=5.0 \mathrm{~Hz}, 1 \mathrm{H}), 3.46-3.34(\mathrm{~m}, 2 \mathrm{H}), 3.30(\mathrm{dd}$, $J=10.8$ and $5.9 \mathrm{~Hz}, 1 \mathrm{H}), 2.78(\mathrm{dd}, J=12.8$ and $4.4 \mathrm{~Hz}, 1 \mathrm{H}), 2.60(\mathrm{dd}, J=12.8$ and $6.1 \mathrm{~Hz}, 1 \mathrm{H}), 1.45(\mathrm{~s}$, $\left.3 \mathrm{H}, \mathrm{NH}, \mathrm{NH}_{2}\right) ;{ }^{13} \mathrm{C} \mathrm{NMR}\left(125 \mathrm{MHz}, \mathrm{CDCl}_{3}\right): \delta$ (ppm): 138.31, 138.30, 138.27, 128.4, 128.3, 128.1, 127.9, $127.7,127.69,127.66,79.4,78.6,73.2,72.1,71.7,71.5,62.9,61.1,44.9$; HRMS (ESI): calcd for $\mathrm{C}_{27} \mathrm{H}_{33} \mathrm{O}_{3} \mathrm{~N}_{2}{ }^{+}$ $\left[\mathrm{M}+\mathrm{H}^{+}\right]$433.2486, found 433.2479.

Data for (2S,3S,4R,5R)-2-(aminomethyl)-3,4-bis(benzyloxy)-5-(benzyloxymethyl)pyrrolidine (H-3): Colourless syrup, $380 \mathrm{mg}$, 88\% yield from hydroxylamine $\mathbf{H}-2$ (450 mg, $1.01 \mathrm{mmol}) ;[\alpha]_{\mathrm{D}}{ }^{23}-15.4$ (c 1.0 in $\left.\mathrm{CH}_{2} \mathrm{Cl}_{2}\right) ; v_{\max } / \mathrm{cm}^{-1}$ : $3061(\mathrm{~m}), 3029(\mathrm{~m}), 2861(\mathrm{~s}), 1496(\mathrm{~m}), 1453(\mathrm{~s}), 1362(\mathrm{~m}), 1122(\mathrm{~s}), 1094(\mathrm{~s}), 1027$ (m), $736(\mathrm{~s}), 697(\mathrm{vs}) ;{ }^{1} \mathrm{H}$ NMR $\left(400 \mathrm{MHz}, \mathrm{CDCl}_{3}\right) \delta(\mathrm{ppm}): 7.34-7.23(\mathrm{~m}, 15 \mathrm{H}), 4.60-4.43(\mathrm{~m}, 6 \mathrm{H}), 3.75$ $(\mathrm{t}, J=5.0 \mathrm{~Hz}, 1 \mathrm{H}), 3.55(\mathrm{t}, J=5.9 \mathrm{~Hz}, 1 \mathrm{H}), 3.49(\mathrm{dd}, J=9.9$ and $5.0 \mathrm{~Hz}, 1 \mathrm{H}), 3.46-3.34(\mathrm{~m}, 2 \mathrm{H}), 3.30(\mathrm{dd}$, $J=10.8$ and $5.9 \mathrm{~Hz}, 1 \mathrm{H}), 2.78(\mathrm{dd}, J=12.8$ and $4.4 \mathrm{~Hz}, 1 \mathrm{H}), 2.60(\mathrm{dd}, J=12.8$ and $6.1 \mathrm{~Hz}, 1 \mathrm{H}), 1.43(\mathrm{~s}$, $\left.3 \mathrm{H}, \mathrm{NH}, \mathrm{NH}_{2}\right) ;{ }^{13} \mathrm{C} \mathrm{NMR}\left(125 \mathrm{MHz}, \mathrm{CDCl}_{3}\right) \delta$ (ppm): 138.3, 138.29, 138.26, 128.4, 128.3, 128.1, 127.9, 127.7, 127.69, 127.65, 79.4, 78.6, 73.2, 72.1, 71.7, 71.5, 62.9, 61.1, 44.9; HRMS (ESI): calcd for $\mathrm{C}_{27} \mathrm{H}_{33} \mathrm{O}_{3} \mathrm{~N}_{2}{ }^{+}$ $\left[\mathrm{M}+\mathrm{H}^{+}\right]$433.2486, found 433.2479.

\subsubsection{Synthesis of (2S,3S,4S,5S)-3,4-bis(benzyloxy)-5-(benzyloxymethyl)-2-cyano-pyrrolidine (A-6)}

To a suspension of iron powder (560 mg, $10.00 \mathrm{mmol}$, used as received) in acetic acid was added Copper (II) acetate ( $20 \mathrm{mg}, 0.10 \mathrm{mmol})$, and the mixture was stirred at room temperature for 5-10 min until the bluish green suspension turned into reddish brown. The solution of hydroxylamine A-2 $(450 \mathrm{mg}, 1.01 \mathrm{mmol})$ in acetic acid $(10 \mathrm{~mL})$ was added, and the reaction mixture was stirred at room temperature overnight. Solvent was removed in vacuo, the residue was neutralized by aqueous $\mathrm{NaHCO}_{3}$ and filtered. The resulting filtrate was extracted with EtOAc $(3 \times 50 \mathrm{~mL})$, then organic phases were combined, dried over $\mathrm{MgSO}_{4}$ and concentrated under reduced pressure. Purification by flash chromatography on silica gel (petroleum ether/EtOAc $=3 / 1$ ) afforded pyrrolidine A-6 (light yellow syrup, $389 \mathrm{mg}$, 91\% yield). Data for (2S,3S,4S,5S)-3,4-bis(benzyloxy)-5-(benzyloxymethyl) -2-cyano-pyrrolidine (A-6): $[\alpha]_{\mathrm{D}}{ }^{23}-21.2$ (c 1.0 in $\left.\mathrm{CH}_{2} \mathrm{Cl}_{2}\right) ; v_{\max } / \mathrm{cm}^{-1}$ : $3293(\mathrm{w}), 3030(\mathrm{w}), 2926(\mathrm{~m})$, 2869 (m), 2246 (w), 1717 (m), 1661 (s), 1453 (s), 1397(m), 1149 (s), 1102 (s), 1027 (m), 736 (s), 697 (vs); ${ }^{1} \mathrm{H}$ 
NMR (500 MHz, CDCl $) \delta(\mathrm{ppm}): 7.36-7.25(\mathrm{~m}, 15 \mathrm{H}), 4.59-4.45(\mathrm{~m}, 6 \mathrm{H}), 4.27(\mathrm{t}, J=3.1 \mathrm{~Hz}, 1 \mathrm{H}), 3.76(\mathrm{~d}$, $J=2.9 \mathrm{~Hz}, 1 \mathrm{H}), 3.83(\mathrm{dd}, J=5.5$ and $3.3 \mathrm{~Hz}, 1 \mathrm{H}), 3.59(\mathrm{q}, J=6.5 \mathrm{~Hz}, 1 \mathrm{H}), 3.49-3.45(\mathrm{~m}, 2 \mathrm{H}), 2.45(\mathrm{br}, 1 \mathrm{H}$, $\mathrm{NH}) ;{ }^{13} \mathrm{C}$ NMR $\left(125 \mathrm{MHz}, \mathrm{CDCl}_{3}\right) \delta$ (ppm): 137.7, 1377.5, 136.7, 128.6, 128.4, 128.2, 128.0, 127.87, 127.82, $127.78,127.74,119.3,87.4,84.1,73.3,72.5,72.2,69.7,62.1,51.9$; HRMS (ESI): calcd for $\mathrm{C}_{27} \mathrm{H}_{29} \mathrm{O}_{3} \mathrm{~N}_{2}{ }^{+}$ $\left[\mathrm{M}+\mathrm{H}^{+}\right]$429.2173, found 429.2181.

\subsubsection{Synthesis of tert-butyl-(2S,3S,4S,5S)-2-(aminomethyl)-3,4-bis(benzyloxy)-5-(benzyloxy methyl)pyrrolidine-1-carboxylate (A-8)}

The mixture of compound A-6 (389 mg, $0.91 \mathrm{mmol})$ and $\mathrm{Et}_{3} \mathrm{~N}(190 \mu \mathrm{L}, 1.37 \mathrm{mmol})$ in dichloromethane $(5 \mathrm{~mL})$ was cooled by an ice-water bath, and $\mathrm{Boc}_{2} \mathrm{O}(298 \mathrm{mg}, 1.37 \mathrm{mmol})$ was added. After stirring overnight at room temperature, the reaction was quenched by aqueous $\mathrm{NaHCO}_{3}$. The solution was then extracted with dichloromethane $(3 \times 10 \mathrm{~mL})$, then organic phases were combined, dried over $\mathrm{MgSO}_{4}$ and concentrated in vacuo to give intermediate A-7 as a light yellow syrup (479 mg, 99\% yield). The crude A-7 was directly dissolved in methanol (5 mL), and Raney Ni (500 mg, 60\%) was added. The suspension was stirred under hydrogen atmosphere for $24 \mathrm{~h}$ when TLC showed part of intermediate A-7 remained unreacted. Longer reaction time did not lead to any further change. Hydrogen was then replaced by nitrogen, and catalyst was removed from the reaction mixture. The filtrate was concentrated in vacuo to afford a colourless oil, which was purified by flash chromatography (silica gel, dichloromethane/methanol $=50: 1$ ) to give compound A-8 (colourless syrup, $126 \mathrm{mg}$, 26\% yield). Data for tert-butyl-(2S,3S,4S,5S)-2-(aminomethyl)-3,4-bis(benzyloxy)-5 -(benzyloxymethyl)pyrrolidine-1-carboxylate (A-8): $[\alpha]_{\mathrm{D}}{ }^{23}-12.7\left(\right.$ c 0.6 in $\left.\mathrm{CH}_{2} \mathrm{Cl}_{2}\right) ; v_{\max } / \mathrm{cm}^{-1}: 3029$ (w), $2973(\mathrm{~m}), 2929(\mathrm{~m}), 1688(\mathrm{~s}), 1453(\mathrm{~m}), 1391(\mathrm{vs}), 1367(\mathrm{~s}), 1160$ (s), $1108(\mathrm{~s}), 1027(\mathrm{~m}), 734(\mathrm{~s}), 697(\mathrm{~s})$; ${ }^{1} \mathrm{H}$ NMR $\left(500 \mathrm{MHz}, \mathrm{CDCl}_{3}\right) \delta(\mathrm{ppm}): 7.37-7.23(\mathrm{~m}, 15 \mathrm{H}), 4.77-4.41(\mathrm{~m}, 6 \mathrm{H}), 4.18(\mathrm{~d}, J=7.7 \mathrm{~Hz}, 1 \mathrm{H})$, 4.15-4.10 (m, 2H), 3.99-3.94 (m, 1H), 3.94-3.87 (m, 1H), $3.57(\mathrm{~d}, J=6.5 \mathrm{~Hz}, 1 \mathrm{H}), 3.24(\mathrm{~d}, J=13.0 \mathrm{~Hz}, 1 \mathrm{H})$, $2.87(\mathrm{dd}, J=12.9$ and $8.5 \mathrm{~Hz}, 1 \mathrm{H}), 1.39(\mathrm{~s}, 9 \mathrm{H}) ;{ }^{13} \mathrm{C} \mathrm{NMR}\left(125 \mathrm{MHz}, \mathrm{CDCl}_{3}\right) \delta(\mathrm{ppm}): 156.7,138.3,137.8$, 137.7, 128.38, 128.33, 128.2, 127.89, 127.84, 127.69, 127.60, 127.5, 127.4, 82.1, 80.3, 77.2, 73.2, 72.4, 72.1, 69.5, 60.3, 59.2, 44.4, 28.2; HRMS (ESI): calcd for $\mathrm{C}_{32} \mathrm{H}_{40} \mathrm{O}_{5} \mathrm{~N}_{2}{ }^{+}\left[\mathrm{M}+\mathrm{H}^{+}\right]$533.3010, found 533.3013.

\subsubsection{Synthesis of tert-butyl-(2S,3S,4S,5S)-2- $N$-acetylaminomethyl-3,4-bis(benzyloxy)-5-} (benzyloxymethyl)pyrrolidine-1-carboxylate (A-9)

Compound A-8 (126 mg, $0.24 \mathrm{mmol})$ was dissolved in dichloromethane $(5 \mathrm{~mL})$, followed by $\mathrm{Ac}_{2} \mathrm{O}$ $(28 \mu \mathrm{L}, 0.29 \mathrm{mmol})$ and catalytic amount of DMAP. The solution was stirred at room temperature for 3-4 h, when TLC showed completion of the reaction. The solution was quenched by aqueous $\mathrm{NaHCO}_{3}$, and extracted with dichloromethane $(3 \times 10 \mathrm{~mL})$. The organic phases were combined, dried over $\mathrm{MgSO}_{4}$ and concentrated in vacuo. The residue was purified by flash chromatography (silica gel, petroleum ether/EtOAc $=1 / 1$ ) to afford compound A-9 (colourless syrup, $124 \mathrm{mg}, 93 \%$ yield). Data for tert-butyl-(2S,3S,4S,5S)-2- $\mathrm{N}$-acetylaminomethyl-3,4-bis(benzyloxy)-5-(benzyloxy methyl)pyrrolidine-1-carboxylate (A-9): $[\alpha]_{\mathrm{D}}^{23}-19.1\left(c 1.0\right.$ in $\left.\mathrm{CH}_{2} \mathrm{Cl}_{2}\right) ; v_{\max } / \mathrm{cm}^{-1}: 3299(\mathrm{w}), 3029$ (w), $2927(\mathrm{~m}), 1690(\mathrm{vs}), 1682(\mathrm{~m}), 1453(\mathrm{~m}), 1390(\mathrm{~s}), 1366(\mathrm{~s}), 1274(\mathrm{~m}), 1174(\mathrm{~m}), 1096(\mathrm{~s}), 1027(\mathrm{~m}), 735$ (m), $697(\mathrm{~m}){ }^{1}{ }^{1} \mathrm{H}$ NMR $\left(500 \mathrm{MHz}, \mathrm{CDCl}_{3}\right) \delta$ (ppm): 7.33-7.25 (m, 15H), 6.64 (br, 1H, NHCO), 4.75-4.44 $(\mathrm{m}, 6 \mathrm{H}), 4.18(\mathrm{dd}, J=8.8$ and $6.1 \mathrm{~Hz}, 1 \mathrm{H}), 4.15-4.09(\mathrm{~m}, 2 \mathrm{H}), 3.95-3.85(\mathrm{~m}, 2 \mathrm{H}), 3.57(\mathrm{~d}, J=6.5 \mathrm{~Hz}, 1 \mathrm{H})$, 3.46-3.37 (m, 1H), 3.19-3.10 (m, 1H), $1.87(\mathrm{~s}, 1 \mathrm{H}), 1.39(\mathrm{~s}, 9 \mathrm{H}) ;{ }^{13} \mathrm{C} \mathrm{NMR}\left(125 \mathrm{MHz}, \mathrm{CDCl}_{3}\right) \delta(\mathrm{ppm})$ : $170.3,155.5,138.4,138.1,128.3,128.2,127.8,127.7,127.68,127.62,127.4,80.7,79.8,77.8,73.2,72.5,72.4$, 70.0, 61.7, 58.6, 43.0, 28.3, 23.2; HRMS (ESI): calcd for $\mathrm{C}_{34} \mathrm{H}_{43} \mathrm{O}_{6} \mathrm{~N}_{2}{ }^{+}\left[\mathrm{M}+\mathrm{H}^{+}\right]$575.3116, found 575.3118.

3.3.6. General Procedure for Synthesis of Compounds A-5, B-5, C-5, D-5, E-5a, E-5b, F-5a, F-5b, G-5 and $\mathbf{H - 5}$ with A-5 as an Example

To the solution of compound A-3 $(215 \mathrm{mg}, 0.50 \mathrm{mmol})$ in dichloromethane $(10 \mathrm{~mL})$ was added $N, N^{\prime}, N^{\prime \prime}, N^{\prime \prime \prime}$-tetraacetylglycoluril (170 mg, $0.55 \mathrm{mmol}$ ). The solution was refluxed for $3-5 \mathrm{~h}$, when TLC showed disapearance of the starting material. The reaction was quenched by aqueous $\mathrm{NaHCO}_{3}$, and 
extracted with dichloromethane $(3 \times 10 \mathrm{~mL})$. The organic phases were combined, dried over $\mathrm{MgSO}_{4}$ and concentrated under reduced pressure. The crude product was purified by flash chromatography (silica gel, dichloromethane/methanol = 50:1) to give compound A-5 (colourless syrup, $208 \mathrm{mg}, 88 \%$ yield). Data for (2S,3S,4S,5S)-2- $\mathrm{N}$-acetylaminomethyl-3,4-bis(benzyloxy)-5- (benzyloxymethyl)pyrrolidine (A-5): $[\alpha]_{\mathrm{D}}^{23}-7.7\left(\mathrm{c} 0.9\right.$ in $\left.\mathrm{CH}_{2} \mathrm{Cl}_{2}\right) ; v_{\max } / \mathrm{cm}^{-1}$ : $3292(\mathrm{w}), 3030(\mathrm{~m}), 2860(\mathrm{~m}), 1656(\mathrm{~s}), 1453(\mathrm{~m}), 1363$ (m), 1094 (s), 1027 (m), 737 (s), 697 (vs); ${ }^{1} \mathrm{H}$ NMR (500 MHz, CDCl $) \delta(\mathrm{ppm}):$ 7.34-7.25 (m, 15H), 6.08 (br, 1H, NHCO), 4.55-4.49 (m, 6H), $3.88(\mathrm{t}, J=3.5 \mathrm{~Hz}, 1 \mathrm{H}), 3.76(\mathrm{t}, J=3.4 \mathrm{~Hz}, 1 \mathrm{H}), 3.54-3.48$ (m, 2H), 3.43-3.38 (m, 1H), 3.37-3.31 (m, 2H), 3.28-3.23 (m, 1H), $2.1(\mathrm{br}, 1 \mathrm{H}, \mathrm{NH}), 1.88(\mathrm{~s}, 3 \mathrm{H}) ;{ }^{13} \mathrm{C}$ NMR (125 MHz, $\mathrm{CDCl}_{3}$ ) $\delta$ (ppm): 170.2, 137.9, 137.83, 137.81, 128.46, 128.44, 127.87, 127.84, 127.81, 127.77, 127.73, 86.8, 85.5, 73.2, 72.0, 71.8, 69.8, 61.9, 61.0, 41.4, 23.2; HRMS (ESI): calcd for $\mathrm{C}_{29} \mathrm{H}_{35} \mathrm{O}_{4} \mathrm{~N}_{2}{ }^{+}$ $\left[\mathrm{M}+\mathrm{H}^{+}\right]$475.2591, found 475.2588.

Data for (2R,3R,4R,5R)-2-N-acetylaminomethyl-3,4-bis(benzyloxy)-5-(benzyloxymethyl) pyrrolidine (B-5) (Ref. [62]): Colourless syrup, $216 \mathrm{mg}, 91 \%$ yield from diamine B-3 (215 mg, $0.50 \mathrm{mmol}) ;[\alpha]_{\mathrm{D}}{ }^{23}$ +11.9 (c 1.0 in $\left.\mathrm{CH}_{2} \mathrm{Cl}_{2}\right) ; v_{\max } / \mathrm{cm}^{-1}: 3283(\mathrm{w}), 3030(\mathrm{~m}), 2859(\mathrm{~m}), 1656(\mathrm{~s}), 1454(\mathrm{~m}), 1363(\mathrm{~m}), 1093$ (s), $1028(\mathrm{~m}), 737$ (s), $697(\mathrm{vs}) ;{ }^{1} \mathrm{H}$ NMR $\left(500 \mathrm{MHz}, \mathrm{CDCl}_{3}\right) \delta(\mathrm{ppm}): 7.34-7.25(\mathrm{~m}, 15 \mathrm{H}), 6.08(\mathrm{br}, 1 \mathrm{H}$, NHCO $), 4.55-4.49(\mathrm{~m}, 6 \mathrm{H}), 3.88(\mathrm{t}, J=3.5 \mathrm{~Hz}, 1 \mathrm{H}), 3.76(\mathrm{t}, J=3.4 \mathrm{~Hz}, 1 \mathrm{H}), 3.54-3.48(\mathrm{~m}, 2 \mathrm{H}), 3.43-3.38$ $(\mathrm{m}, 1 \mathrm{H}), 3.37-3.31(\mathrm{~m}, 2 \mathrm{H}), 3.28-3.23(\mathrm{~m}, 1 \mathrm{H}), 2.16(\mathrm{br}, 1 \mathrm{H}, \mathrm{NH}), 1.88(\mathrm{~s}, 3 \mathrm{H}) ;{ }^{13} \mathrm{C} \mathrm{NMR}(125 \mathrm{MHz}$, $\left.\mathrm{CDCl}_{3}\right) \delta$ (ppm): 170.2, 137.9, 137.83, 137.81, 128.46, 128.44, 127.87, 127.84, 127.81, 127.77, 127.73, 86.8, 85.5, 73.2, 72.0, 71.8, 69.8, 61.9, 61.0, 41.4, 23.2; HRMS (ESI): calcd for $\mathrm{C}_{29} \mathrm{H}_{35} \mathrm{O}_{4} \mathrm{~N}_{2}{ }^{+}\left[\mathrm{M}+\mathrm{H}^{+}\right]$475.2591, found 475.2586 .

Data for (2S,3S,4R,5S)-2- $N$-acetylaminomethyl-3,4-bis(benzyloxy)-5-(benzyloxymethyl) pyrrolidine (C-5): Colourless syrup, $201 \mathrm{mg}$, 85\% yield from diamine C-3 $(215 \mathrm{mg}, 0.50 \mathrm{mmol}) ;[\alpha]_{\mathrm{D}}{ }^{23}+11.9($ c 1.0 in $\left.\mathrm{CH}_{2} \mathrm{Cl}_{2}\right) ; v_{\max } / \mathrm{cm}^{-1}: 3288(\mathrm{w}), 3029(\mathrm{w}), 2924(\mathrm{~m}), 2855(\mathrm{~m}), 1652(\mathrm{~s}), 1453(\mathrm{~m}), 1366(\mathrm{~m}), 1089(\mathrm{~s})$, $1027(\mathrm{~m}), 736(\mathrm{~s}), 697(\mathrm{~s}) ;{ }^{1} \mathrm{H}$ NMR $\left(500 \mathrm{MHz}, \mathrm{CDCl}_{3}\right) \delta(\mathrm{ppm}): 7.35-7.25(\mathrm{~m}, 15 \mathrm{H}), 5.87(\mathrm{br}, 1 \mathrm{H}, \mathrm{NH})$, $4.73-4.49(\mathrm{~m}, 6 \mathrm{H}), 4.00(\mathrm{t}, J=4.0 \mathrm{~Hz}, 1 \mathrm{H}), 3.68-3.62(\mathrm{~m}, 2 \mathrm{H}), 3.58(\mathrm{t}, J=7.5 \mathrm{~Hz}, 1 \mathrm{H}), 3.44-3.38(\mathrm{~m}, 2 \mathrm{H})$, 3.35-3.25 (m, 2H), 2.16 (br, 2H, NH $), 1.89(\mathrm{~s}, 3 \mathrm{H}) ;{ }^{13} \mathrm{C} \mathrm{NMR}\left(125 \mathrm{MHz}, \mathrm{CDCl}_{3}\right) \delta$ (ppm): 170.3, 138.3, 138.0, 137.8, 128.47, 128.42, 128.3, 127.89, 127.88, 127.86, 127.80, 127.7, 127.6, 82.4, 77.6, 73.4, 73.2, 72.5, 69.5, 59.07, 59.04, 42.3, 23.2; HRMS (ESI): calcd for $\mathrm{C}_{29} \mathrm{H}_{35} \mathrm{O}_{4} \mathrm{~N}_{2}{ }^{+}\left[\mathrm{M}+\mathrm{H}^{+}\right]$475.2591, found 475.2588.

Data for (2R,3R,4S,5R)-2-N-acetylaminomethyl-3,4-bis(benzyloxy)-5-(benzyloxymethyl) pyrrolidine (D-5): Colourless syrup, $194 \mathrm{mg}, 82 \%$ yield from diamine $\mathbf{D}-3(215 \mathrm{mg}, 0.50 \mathrm{mmol}) ;[\alpha]_{\mathrm{D}}{ }^{23}-2.5$ (c $1.0 \mathrm{in}$ $\left.\mathrm{CH}_{2} \mathrm{Cl}_{2}\right) ; v_{\max } / \mathrm{cm}^{-1}: 3296(\mathrm{w}), 3028(\mathrm{w}), 2921(\mathrm{~m}), 2855(\mathrm{~m}), 1652(\mathrm{~s}), 1554(\mathrm{~m}), 1453(\mathrm{~s}), 1365(\mathrm{~m}), 1091$ (s), $1027(\mathrm{~m}), 736(\mathrm{~s}), 696(\mathrm{~s}) ;{ }^{1} \mathrm{H}$ NMR $\left(500 \mathrm{MHz}, \mathrm{CDCl}_{3}\right) \delta(\mathrm{ppm}): 7.35-7.25(\mathrm{~m}, 15 \mathrm{H}), 5.87(\mathrm{br}, 1 \mathrm{H}$, $\mathrm{NHCO}), 4.73-4.49(\mathrm{~m}, 6 \mathrm{H}), 4.00(\mathrm{t}, J=4.1 \mathrm{~Hz}, 1 \mathrm{H}), 3.68-3.62(\mathrm{~m}, 2 \mathrm{H}), 3.58(\mathrm{t}, J=7.5 \mathrm{~Hz}, 1 \mathrm{H}), 3.44-3.38$ $(\mathrm{m}, 2 \mathrm{H}), 3.35-3.25(\mathrm{~m}, 2 \mathrm{H}), 2.16(\mathrm{br}, 1 \mathrm{H}, \mathrm{NH}), 1.89(\mathrm{~s}, 3 \mathrm{H}) ;{ }^{13} \mathrm{C} \mathrm{NMR}\left(125 \mathrm{MHz}, \mathrm{CDCl}_{3}\right) \delta(\mathrm{ppm}): 170.3$, 138.4, 138.0, 137.9, 128.49, 128.44, 128.3, 127.9, 127.8, 127.7, 127.6, 82.4, 77.7, 73.4, 73.3, 72.6, 69.5, 59.09, 59.06, 42.3, 23.2; HRMS (ESI): calcd for $\mathrm{C}_{29} \mathrm{H}_{35} \mathrm{O}_{4} \mathrm{~N}_{2}{ }^{+}\left[\mathrm{M}+\mathrm{H}^{+}\right]$475.2591, found 475.2588.

Data for (2R,3R,4R,5S)-2- $N$-acetylaminomethyl-3,4-bis(benzyloxy)-5-(benzyloxymethyl) pyrrolidine (E-5a): Colourless syrup, $213 \mathrm{mg}, 90 \%$ yield from diamine E-3a $(215 \mathrm{mg}, 0.50 \mathrm{mmol}) ;[\alpha]_{\mathrm{D}}{ }^{23}+9.2(c 1.0$ in $\left.\mathrm{CH}_{2} \mathrm{Cl}_{2}\right) ; v_{\max } / \mathrm{cm}^{-1}: 3295(\mathrm{w}), 3030(\mathrm{w}), 2924(\mathrm{~m}), 2855(\mathrm{~m}), 1652$ (s), $1554(\mathrm{~m}), 1493$ (s), $1365(\mathrm{~m})$, 1091 (s), 1027 (m), $736(\mathrm{~s}), 696(\mathrm{~s}) ;{ }^{1} \mathrm{H}$ NMR (500 MHz, $\left.\mathrm{CDCl}_{3}\right) \delta(\mathrm{ppm}): 7.34-7.24(\mathrm{~m}, 15 \mathrm{H}), 6.81(\mathrm{br}, 1 \mathrm{H}$, NHCO), 4.58-4.42 (m, 6H), $4.19(\mathrm{br}, 1 \mathrm{H}, \mathrm{NH}), 3.90(\mathrm{~d}, J=3.9 \mathrm{~Hz}, 1 \mathrm{H}), 3.77(\mathrm{~s}, 1 \mathrm{H}), 3.73(\mathrm{dd}, J=9.3$ and $5.8 \mathrm{~Hz}, 1 \mathrm{H}), 3.68(\mathrm{dd}, J=9.5$ and $6.7 \mathrm{~Hz}, 2 \mathrm{H}), 3.62(\mathrm{dd}, J=10.3$ and $6.0 \mathrm{~Hz}, 1 \mathrm{H}), 3.48-3.43(\mathrm{~m}, 1 \mathrm{H})$, 3.39-3.34 (m, 1H), $1.76(\mathrm{~s}, 3 \mathrm{H}) ;{ }^{13} \mathrm{C} \mathrm{NMR}\left(125 \mathrm{MHz}, \mathrm{CDCl}_{3}\right) \delta(\mathrm{ppm}): 170.6,138.1,137.7,137.6,128.54$, 128.52, 128.4, 127.99, 127.91, 127.8, 127.79, 127.75, 127.6, 85.0, 82.2, 73.5, 71.9, 71.8, 69.6, 62.0, 60.4, 42.4, 22.8; HRMS (ESI): calcd for $\mathrm{C}_{29} \mathrm{H}_{35} \mathrm{O}_{4} \mathrm{~N}_{2}{ }^{+}\left[\mathrm{M}+\mathrm{H}^{+}\right]$475.2591, found 475.2585.

Data for (2S,3R,4R,5S)-2-N-acetylaminomethyl-3,4-bis(benzyloxy)-5-(benzyloxymethyl) pyrrolidine (E-5b): Colourless syrup, $194 \mathrm{mg}$, 82\% yield from diamine E-3b $(215 \mathrm{mg}, 0.50 \mathrm{mmol}) ;[\alpha]_{\mathrm{D}}{ }^{23}-17.2$ (c 1.2 in $\mathrm{CH}_{2} \mathrm{Cl}_{2}$ ); $v_{\text {max }} / \mathrm{cm}^{-1}$ : 3290 (m), 3063 (m), 3030 (m), 2925 (s), 2861 (s), 1651 (vs), 1549 (m), 1453 (s), 
1367 (s), 1092 (vs), 1027 (m), 736 (vs), 697 (vs); ${ }^{1} \mathrm{H}$ NMR (500 MHz, $\left.\mathrm{CDCl}_{3}\right) \delta$ (ppm): 7.36-7.25 (m, 15H), $6.11(\mathrm{br}, 1 \mathrm{H}, \mathrm{NHCO}), 4.56-4.35(\mathrm{~m}, 6 \mathrm{H}), 3.99(\mathrm{~d}, J=1.6 \mathrm{~Hz}, 1 \mathrm{H}), 3.93(\mathrm{~d}, J=3.1 \mathrm{~Hz}, 1 \mathrm{H}), 3.63-3.61(\mathrm{~m}$, $2 \mathrm{H}), 3.57-3.52(\mathrm{~m}, 3 \mathrm{H}), 3.30-3.25(\mathrm{~m}, 1 \mathrm{H}), 2.36(\mathrm{br}, 1 \mathrm{H}, \mathrm{NH}), 1.85(\mathrm{~s}, 3 \mathrm{H}) ;{ }^{13} \mathrm{C} \mathrm{NMR}\left(125 \mathrm{MHz}, \mathrm{CDCl}_{3}\right) \delta$ (ppm): 170.4, 138.1, 137.9, 137.7, 128.6, 128.4, 128.0, 127.89, 127.86, 127.84, 127.7, 82.6, 82.0, 73.4, 72.3, 72.1, 68.8, 58.9, 58.2, 39.6, 23.2; HRMS (ESI): calcd for $\mathrm{C}_{29} \mathrm{H}_{35} \mathrm{O}_{4} \mathrm{~N}_{2}{ }^{+}\left[\mathrm{M}+\mathrm{H}^{+}\right]$475.2591, found 475.2584.

Data for (2S,3S,4S,5R)-2- $N$-acetylaminomethyl-3,4-bis(benzyloxy)-5-(benzyloxymethyl) pyrrolidine (F-5a): Colourless syrup, $206 \mathrm{mg}, 87 \%$ yield from diamine F-3a $(215 \mathrm{mg}, 0.50 \mathrm{mmol}) ;[\alpha]_{\mathrm{D}}{ }^{22}-4.1$ (c 1.0 in $\left.\mathrm{CH}_{2} \mathrm{Cl}_{2}\right)$; $v_{\max } / \mathrm{cm}^{-1}: 3295(\mathrm{w}), 3064(\mathrm{~m}), 3030(\mathrm{~m}), 2924(\mathrm{~m}), 2855(\mathrm{~m}), 1652(\mathrm{~s}), 1554(\mathrm{~m}), 1493$ (m), $1365(\mathrm{~m}), 1091(\mathrm{~s}), 1027(\mathrm{~m}), 736(\mathrm{~s}), 696$ (s); ${ }^{1} \mathrm{H}$ NMR (500 MHz, $\left.\mathrm{CDCl}_{3}\right) \delta(\mathrm{ppm}): 7.34-7.23(\mathrm{~m}$, $15 \mathrm{H}) 6.54(\mathrm{br}, 1 \mathrm{H}, \mathrm{NHCO}), 4.58-4.42(\mathrm{~m}, 6 \mathrm{H}), 3.89(\mathrm{dd}, J=4.1$ and $0.6 \mathrm{~Hz}, 1 \mathrm{H}), 3.74-3.71(\mathrm{~m}, 2 \mathrm{H}), 3.65$ $(\mathrm{dd}, J=8.9$ and $6.9 \mathrm{~Hz}, 1 \mathrm{H}), 3.59(\mathrm{dd}, J=10.0$ and $5.4 \mathrm{~Hz}, 1 \mathrm{H}), 3.44-3.40(\mathrm{~m}, 2 \mathrm{H}), 3.35-3.31(\mathrm{~m}, 1 \mathrm{H})$, 3.19 (br, $1 \mathrm{H}, \mathrm{NH}), 1.73(\mathrm{~s}, 3 \mathrm{H}) ;{ }^{13} \mathrm{C}$ NMR $\left(125 \mathrm{MHz}, \mathrm{CDCl}_{3}\right) \delta$ (ppm): 170.6, 138.1, 137.7, 137.6, 128.54, 128.52, 128.4, 127.99, 127.9, 127.8, 127.79, 127.75, 127.6, 85.0, 82.2, 73.5, 71.9, 71.8, 69.6, 62.0, 60.4, 42.4, 22.8; HRMS (ESI): calcd for $\mathrm{C}_{29} \mathrm{H}_{35} \mathrm{O}_{4} \mathrm{~N}_{2}{ }^{+}\left[\mathrm{M}+\mathrm{H}^{+}\right]$475.2591, found 475.2587.

Data for (2R,3S,4S,5R)-2- $\mathrm{N}$-acetylaminomethyl-3,4-bis(benzyloxy)-5-(benzyloxymethyl) pyrrolidine (F-5b): Colourless syrup, $197 \mathrm{mg}$, 83\% yield from diamine F-3b (215 mg, $0.50 \mathrm{mmol}) ;[\alpha]_{\mathrm{D}}{ }^{22}+20.5(c$ 1.0 in $\left.\mathrm{CH}_{2} \mathrm{Cl}_{2}\right) ; v_{\max } / \mathrm{cm}^{-1}: 3293(\mathrm{~m}), 3063(\mathrm{~m}), 3030(\mathrm{~m}), 2924(\mathrm{~s}), 2861$ (s), 1651 (vs), $1549(\mathrm{~m}), 1453$ (s), 1367 (s), 1092 (vs), 1027 (m), 736 (vs), 697 (vs); ${ }^{1} \mathrm{H}$ NMR (500 MHz, $\mathrm{CDCl}_{3}$ ) $\delta$ (ppm): 7.35-7.24 (m, $15 \mathrm{H}) 6.24(\mathrm{br}, 1 \mathrm{H}, \mathrm{NHCO}), 4.55-4.35(\mathrm{~m}, 6 \mathrm{H}), 3.98(\mathrm{dd}, J=6.0$ and $1.9 \mathrm{~Hz}, 1 \mathrm{H}), 3.92(\mathrm{dd}, J=4.9$ and $1.9 \mathrm{~Hz}, 1 \mathrm{H}), 3.63(\mathrm{~m}, 2 \mathrm{H}), 3.57-3.52(\mathrm{~m}, 3 \mathrm{H}), 3.29-3.23(\mathrm{~m}, 1 \mathrm{H}), 3.12(\mathrm{br}, 1 \mathrm{H}, \mathrm{NH}), 1.84(\mathrm{~s}, 3 \mathrm{H}) ;{ }^{13} \mathrm{C}$ NMR (125 MHz, $\mathrm{CDCl}_{3}$ ) $\delta$ (ppm): 170.4, 138.1, 137.9, 137.7, 128.6, 128.48, 128.42, 128.0, 127.89, 127.86, $127.85,127.7,82.5,82.0,73.4,72.3,72.1,68.9,58.9,58.2,39.6,23.2$; HRMS (ESI): calcd for $\mathrm{C}_{29} \mathrm{H}_{35} \mathrm{O}_{4} \mathrm{~N}_{2}{ }^{+}$ $\left[\mathrm{M}+\mathrm{H}^{+}\right]$475.2591, found 475.2585.

Data for $(2 R, 3 R, 4 S, 5 S)$-2- $N$-acetylaminomethyl-3,4-bis(benzyloxy)-5-(benzyloxymethyl) pyrrolidine (G-5): Colourless syrup, $204 \mathrm{mg}$, 86\% yield from diamine G-3 $(215 \mathrm{mg}, 0.50 \mathrm{mmol}) ;[\alpha]_{\mathrm{D}}{ }^{23}+7.5($ c $1.0 \mathrm{in}$ $\left.\mathrm{CH}_{2} \mathrm{Cl}_{2}\right) ; v_{\max } / \mathrm{cm}^{-1}: 3293(\mathrm{~m}), 3062(\mathrm{~m}), 3029(\mathrm{~m}), 2924(\mathrm{~s}), 2861(\mathrm{~s}), 1653(\mathrm{vs}), 1539(\mathrm{~m}), 1453(\mathrm{~s}), 1365$ (m), 1100 (vs), 1027 (m), 736 (s), 697 (vs); $\left.{ }^{1} \mathrm{H} \mathrm{NMR} \mathrm{(500} \mathrm{MHz,} \mathrm{CDCl}_{3}\right) \delta(\mathrm{ppm}): 7.34-7.24(\mathrm{~m}, 15 \mathrm{H}), 6.12$ (br, 1H, NHCO), 4.58-4.45 (m, 6H), $3.77(\mathrm{t}, J=4.7 \mathrm{~Hz}, 1 \mathrm{H}), 3.60(\mathrm{t}, J=5.5 \mathrm{~Hz}, 1 \mathrm{H}), 3.50(\mathrm{q}, J=4.4 \mathrm{~Hz}$, 1H), 3.47-3.38 (m, 4H), 3.18-3.13 (m, 1H), $2.14(\mathrm{br}, 1 \mathrm{H}, \mathrm{NH}), 1.74(\mathrm{~s}, 3 \mathrm{H}) ;{ }^{13} \mathrm{C} \mathrm{NMR}\left(125 \mathrm{MHz}, \mathrm{CDCl}_{3}\right) \delta$ (ppm): 170.2, 138.0, 137.9, 128.47, 128.40, 128.1, 128.0, 127.86, 127.80, 127.7, 80.0, 78.2, 73.3, 72.0, 71.6, 61.0, 59.6, 42.3, 23.0; HRMS (ESI): calcd for $\mathrm{C}_{29} \mathrm{H}_{35} \mathrm{O}_{4} \mathrm{~N}_{2}{ }^{+}\left[\mathrm{M}+\mathrm{H}^{+}\right]$475.2591, found 475.2587.

Data for (2S,3S,4R,5R)-2- $\mathrm{N}$-acetylaminomethyl-3,4-bis(benzyloxy)-5-(benzyloxymethyl) pyrrolidine (H-5): Colourless syrup, $192 \mathrm{mg}, 81 \%$ yield from diamine $\mathbf{H}-3(215 \mathrm{mg}, 0.50 \mathrm{mmol}) ;[\alpha]_{\mathrm{D}}{ }^{23}-1.6$ (c $1.2 \mathrm{in}$ $\left.\mathrm{CH}_{2} \mathrm{Cl}_{2}\right) ; v_{\max } / \mathrm{cm}^{-1}$ : $3304(\mathrm{~m}), 3062(\mathrm{~m}), 3030(\mathrm{~m}), 2865(\mathrm{~s}), 1651$ (vs), $1549(\mathrm{~m}), 1453(\mathrm{~s}), 1365(\mathrm{~m}), 1099$ (vs), 1027 (m), 736 (vs), 697 (vs); ${ }^{1} \mathrm{H}$ NMR (500 MHz, $\left.\mathrm{CDCl}_{3}\right) \delta(\mathrm{ppm}): 7.33-7.24$ (m, 15H), 6.11 (br, 1H, $\mathrm{NHCO}), 4.57-4.43(\mathrm{~m}, 6 \mathrm{H}), 3.76(\mathrm{t}, J=4.8 \mathrm{~Hz}, 1 \mathrm{H}), 3.59(\mathrm{t}, J=5.6 \mathrm{~Hz}, 1 \mathrm{H}), 3.49(\mathrm{dd}, J=8.9$ and $4.4 \mathrm{~Hz}$, $1 \mathrm{H}), 3.47-3.37(\mathrm{~m}, 4 \mathrm{H}), 3.18-3.13(\mathrm{~m}, 1 \mathrm{H}), 2.14(\mathrm{br}, 1 \mathrm{H}, \mathrm{NH}), 1.74(\mathrm{~s}, 3 \mathrm{H}) ;{ }^{13} \mathrm{C} \mathrm{NMR}\left(125 \mathrm{MHz}, \mathrm{CDCl}_{3}\right)$ : $\delta$ (ppm): 170.2, 138.0, 137.9, 128.47, 128.40, 128.1, 128.0, 127.86, 127.80, 127.7, 80.0, 78.2, 73.3, 72.0, 71.6, 61.0, 59.6, 42.3, 23.0; HRMS (ESI): calcd for $\mathrm{C}_{29} \mathrm{H}_{35} \mathrm{O}_{4} \mathrm{~N}_{2}{ }^{+}\left[\mathrm{M}+\mathrm{H}^{+}\right]$475.2591, found 475.2588.

3.3.7. General Procedure for Synthesis of 1-N-Acetylamino Derivatives (A-10, 4.HCl, C-10, D-10, E-10a, E-10b, F-10a, F-10b, G-10 and H-10) and 1-Amino Derivatives (A-11, B-11, C-11, D-11, E-11a, E-11b, F-11a, F-11b, G-11 and H-11) with A-10 as an Example

To a stirred solution of A-5 (95 mg, $0.20 \mathrm{mmol})$ and $3 \mathrm{~N} \mathrm{HCl}(0.5 \mathrm{~mL})$ in $\mathrm{MeOH}(10 \mathrm{~mL})$ was added $\mathrm{Pd} / \mathrm{C}(10 \mathrm{wt} \%, 30 \mathrm{mg})$ under $\mathrm{Ar}$ atmosphere and the reaction mixture was stirred under $\mathrm{H}_{2}$ atmosphere for $8 \mathrm{~h}$. Then the catalyst was filtered and the solvent was removed under reduced pressure to afford compound A-10 (colourless syrup, $47 \mathrm{mg}, 99 \%$ yield). Data for 1-N-acetylamino-2,5-imino-1,2,5-trideoxy-L-mannitol hydrochloride (A-10) (Ref. [16], reported 
as free base): $[\alpha]_{\mathrm{D}}{ }^{23}-30.4(\mathrm{c} 0.5 \mathrm{in} \mathrm{MeOH}) ; v_{\max } / \mathrm{cm}^{-1}: 3290$ (vs), $2932(\mathrm{~s}), 1646(\mathrm{~m}), 1550(\mathrm{~m}), 1369$ (m), $1132(\mathrm{~m}), 1041(\mathrm{~m}) ;{ }^{1} \mathrm{H}$ NMR $\left(500 \mathrm{MHz}, \mathrm{D}_{2} \mathrm{O}\right) \delta(\mathrm{ppm}): 4.25(\mathrm{~s}, 1 \mathrm{H}),, 4.11(\mathrm{~s}, 1 \mathrm{H}), 3.98(\mathrm{dd}, J=12.0$ $\mathrm{Hz}$ and $4.6 \mathrm{~Hz}, 1 \mathrm{H}), 3.95-3.91(\mathrm{~m}, 1 \mathrm{H}), 3.86(\mathrm{dd}, J=11.8 \mathrm{~Hz}$ and $8.7 \mathrm{~Hz}, 1 \mathrm{H}), 3.66(\mathrm{~d}, J=6.8 \mathrm{~Hz}, 2 \mathrm{H})$, 3.63-3.61 (m, 1H), $2.04\left(\mathrm{~s}, 3 \mathrm{H}, \mathrm{COCH}_{3}\right) ;{ }^{13} \mathrm{C} \mathrm{NMR}\left(125 \mathrm{MHz}, \mathrm{D}_{2} \mathrm{O}\right): \delta(\mathrm{ppm}): 175.2,75.7,74.6,67.5,61.5$, 59.2, 35.8, 21.7; HRMS (ESI): calcd for $\mathrm{C}_{8} \mathrm{H}_{17} \mathrm{O}_{4} \mathrm{~N}_{2}{ }^{+}\left[\mathrm{M}+\mathrm{H}^{+}\right]$205.1183, found 205.1181.

Data for 1- $\mathrm{N}$-acetylamino-2,5-imino-1,2,5-trideoxy-D-mannitol hydrochloride (4.HCl) (Ref. [16,62], reported as free base): Colourless syrup, $50 \mathrm{mg}$, 99\% yield from compound $\mathbf{B}-\mathbf{5}$ (101 $\mathrm{mg}, 0.21 \mathrm{mmol})$; $[\alpha]_{\mathrm{D}}^{25}+34.6(c 0.5$ in $\mathrm{MeOH}) ; v_{\max } / \mathrm{cm}^{-1}: 3297(\mathrm{vs}), 2937(\mathrm{~s}), 1644(\mathrm{~m}), 1550(\mathrm{~m}), 1369(\mathrm{~m}), 1132(\mathrm{~m})$, $1042(\mathrm{~m}) ;{ }^{1} \mathrm{H}$ NMR $\left(500 \mathrm{MHz}, \mathrm{D}_{2} \mathrm{O}\right) \delta(\mathrm{ppm}): 4.22(\mathrm{~d}, J=2.0 \mathrm{~Hz}, 1 \mathrm{H}), 4.09(\mathrm{t}, J=1.6 \mathrm{~Hz}, 1 \mathrm{H}), 3.95(\mathrm{dd}$, $J=12.1 \mathrm{~Hz}$ and $4.8 \mathrm{~Hz}, 1 \mathrm{H}), 3.91(\mathrm{dt}, J=6.9 \mathrm{~Hz}$ and $3.7 \mathrm{~Hz}, 1 \mathrm{H}), 3.83(\mathrm{dd}, J=12.1 \mathrm{~Hz}$ and $8.6 \mathrm{~Hz}, 1 \mathrm{H})$, $3.66(\mathrm{~d}, J=6.8 \mathrm{~Hz}, 2 \mathrm{H}), 3.61-3.58(\mathrm{~m}, 1 \mathrm{H}), 2.01\left(\mathrm{~s}, 3 \mathrm{H}, \mathrm{COCH}_{3}\right) ;{ }^{13} \mathrm{C} \mathrm{NMR}\left(125 \mathrm{MHz}, \mathrm{D}_{2} \mathrm{O}\right) \delta(\mathrm{ppm})$ : 175.2, 75.7, 74.6, 67.5, 61.5, 59.2, 35.8, 21.7; HRMS (ESI): calcd for $\mathrm{C}_{8} \mathrm{H}_{17} \mathrm{O}_{4} \mathrm{~N}_{2}{ }^{+}\left[\mathrm{M}+\mathrm{H}^{+}\right]$205.1183, found 205.1183 .

Data for 1- $N$-acetylamino-2,5-imino-1,2,5-trideoxy-L-altritol hydrochloride (C-10) (Ref. [17], reported as free base): Colourless syrup, $45 \mathrm{mg}, 99 \%$ yield from compound C-5 (90 mg, $0.19 \mathrm{mmol}) ;[\alpha]_{\mathrm{D}}{ }^{22}-18.0$ (c 0.7 in MeOH); $v_{\max } / \mathrm{cm}^{-1}: 3291$ (vs), 2936 (s), 1635 (m), 1550 (m), 1419 (m),1369 (m), 1136 (m), 1042 $(\mathrm{m}) ;{ }^{1} \mathrm{H}$ NMR $\left(500 \mathrm{MHz}, \mathrm{D}_{2} \mathrm{O}\right) \delta(\mathrm{ppm}): 4.33(\mathrm{~s}, 1 \mathrm{H}), 4.23(\mathrm{dd}, J=9.3 \mathrm{~Hz}$ and $3.7 \mathrm{~Hz}, 1 \mathrm{H}), 3.99(\mathrm{dd}$, $J=12.0 \mathrm{~Hz}$ and $4.8 \mathrm{~Hz}, 1 \mathrm{H}), 3.90(\mathrm{dd}, J=11.6 \mathrm{~Hz}$ and $8.4 \mathrm{~Hz}, 1 \mathrm{H}), 3.82-3.80(\mathrm{~m}, 1 \mathrm{H}), 3.72-3.66(\mathrm{~m}, 2 \mathrm{H})$, $3.59(\mathrm{dd}, J=15.3 \mathrm{~Hz}$ and $7.7 \mathrm{~Hz}, 1 \mathrm{H}), 2.04\left(\mathrm{~s}, 3 \mathrm{H}, \mathrm{COCH}_{3}\right) ;{ }^{13} \mathrm{C} \mathrm{NMR}\left(125 \mathrm{MHz}, \mathrm{D}_{2} \mathrm{O}\right) \delta(\mathrm{ppm}): 176.0$, 72.5, 69.8, 62.0, 60.5, 57.4, 38.9, 21.6; HRMS (ESI): calcd for $\mathrm{C}_{8} \mathrm{H}_{17} \mathrm{O}_{4} \mathrm{~N}_{2}{ }^{+}\left[\mathrm{M}+\mathrm{H}^{+}\right]$205.1183, found 205.1186 .

Data for 1-N-acetylamino-2,5-imino-1,2,5-trideoxy-D-altritol hydrochloride (D-10) (Ref. [16,61], reported as free base): Colourless syrup, $41 \mathrm{mg}, 99 \%$ yield from compound D-5 (82 mg, $0.17 \mathrm{mmol}$ ); $[\alpha]_{\mathrm{D}}^{22}+21.3(c 0.4$ in $\mathrm{MeOH}) ; v_{\max } / \mathrm{cm}^{-1}: 3296(\mathrm{vs}), 2936(\mathrm{~s}), 1635(\mathrm{~m}), 1550(\mathrm{~m}), 1419(\mathrm{~m}), 1371(\mathrm{~m})$, $1136(\mathrm{~m}), 1042(\mathrm{~m}) ;{ }^{1} \mathrm{H}$ NMR $\left(500 \mathrm{MHz}, \mathrm{D}_{2} \mathrm{O}\right) \delta(\mathrm{ppm}) 4.33(\mathrm{t}, J=3.2 \mathrm{~Hz}, 1 \mathrm{H}), 4.23(\mathrm{dd}, J=9.3 \mathrm{~Hz}$ and $3.7 \mathrm{~Hz}, 1 \mathrm{H}), 3.99(\mathrm{dd}, J=12.0 \mathrm{~Hz}$ and $4.8 \mathrm{~Hz}, 1 \mathrm{H}), 3.90(\mathrm{dd}, J=11.6 \mathrm{~Hz}$ and $8.4 \mathrm{~Hz}, 1 \mathrm{H}), 3.82-3.80(\mathrm{~m}$, $1 \mathrm{H}), 3.72-3.66(\mathrm{~m}, 2 \mathrm{H}), 3.59(\mathrm{dd}, J=15.3 \mathrm{~Hz}$ and $7.75 \mathrm{~Hz}, 1 \mathrm{H}), 2.04\left(\mathrm{~s}, 3 \mathrm{H}, \mathrm{COCH}_{3}\right) ;{ }^{13} \mathrm{C} \mathrm{NMR}(125 \mathrm{MHz}$, $\left.\mathrm{D}_{2} \mathrm{O}\right) \delta(\mathrm{ppm}): 176.0,72.5,69.8,62.0,60.5,57.4,38.9,21.6$; HRMS (ESI): calcd for $\mathrm{C}_{8} \mathrm{H}_{17} \mathrm{O}_{4} \mathrm{~N}_{2}{ }^{+}\left[\mathrm{M}+\mathrm{H}^{+}\right]$ 205.1183, found 205.1185.

Data for 1- $\mathrm{N}$-acetylamino-2,5-imino-1,2,5-trideoxy-D-glucitol hydrochloride (E-10a) (Ref. [63], reported as free base): Colourless syrup, $46 \mathrm{mg}$, 99\% yield from compound E-5a (91 mg, $0.19 \mathrm{mmol})$; $[\alpha]_{\mathrm{D}}^{25}+36.9(\mathrm{c} 0.85$ in $\mathrm{MeOH}) ; v_{\max } / \mathrm{cm}^{-1}: 3292(\mathrm{vs}), 2935(\mathrm{~s}), 1645(\mathrm{~m}), 1550(\mathrm{~m}), 1369(\mathrm{~m}), 1132(\mathrm{~m})$, $1045(\mathrm{~m}) ;{ }^{1} \mathrm{H}$ NMR $\left(400 \mathrm{MHz}, \mathrm{D}_{2} \mathrm{O}\right) \delta(\mathrm{ppm}): 4.27(\mathrm{~s}, 1 \mathrm{H}), 4.12(\mathrm{~s}, 1 \mathrm{H}), 3.98(\mathrm{~d}, J=9.4 \mathrm{~Hz}, 1 \mathrm{H}), 3.94-3.88$ $(\mathrm{m}, 2 \mathrm{H}), 3.68-3.57(\mathrm{~m}, 3 \mathrm{H}), 2.01\left(\mathrm{~s}, 3 \mathrm{H}, \mathrm{COCH}_{3}\right) ;{ }^{13} \mathrm{C} \mathrm{NMR}\left(125 \mathrm{MHz}, \mathrm{D}_{2} \mathrm{O}\right) \delta(\mathrm{ppm}): 175.8,76.8,74.5$, 65.1, 63.0, 57.0, 39.4, 21.6; HRMS (ESI): calcd for $\mathrm{C}_{8} \mathrm{H}_{17} \mathrm{O}_{4} \mathrm{~N}_{2}{ }^{+}\left[\mathrm{M}+\mathrm{H}^{+}\right]$205.1183, found 205.1182.

Data for 1-N-acetylamino-2,5-imino-1,2,5-trideoxy-L-iditol hydrochloride (E-10b) (Ref. [64], reported as free base): Colourless syrup, $52 \mathrm{mg}$, 99\% yield from compound E- $5 \mathbf{b}(104 \mathrm{mg}, 0.22 \mathrm{mmol}) ;[\alpha]_{\mathrm{D}}{ }^{25}$ -9.6 (c 1.2 in $\mathrm{MeOH}) ; v_{\max } / \mathrm{cm}^{-1}$ : $3292(\mathrm{vs}), 2936(\mathrm{~s}), 1651(\mathrm{~m}), 1553(\mathrm{~m}), 1370(\mathrm{~m}), 1136(\mathrm{~m}), 1042(\mathrm{~m})$; ${ }^{1} \mathrm{H}$ NMR $\left(500 \mathrm{MHz}, \mathrm{D}_{2} \mathrm{O}\right) \delta(\mathrm{ppm}): 4.35(\mathrm{~d}, J=3.3 \mathrm{~Hz}, 1 \mathrm{H}), 4.28(\mathrm{~d}, J=2.0 \mathrm{~Hz}, 1 \mathrm{H}), 4.01-3.95(\mathrm{~m}, 3 \mathrm{H})$, $3.87(\mathrm{dd}, J=11.9$ and $8.3 \mathrm{~Hz}, 1 \mathrm{H}), 3.60(\mathrm{~d}, J=6.8 \mathrm{~Hz}, 2 \mathrm{H}), 2.01\left(\mathrm{~s}, 3 \mathrm{H}, \mathrm{COCH}_{3}\right) ;{ }^{13} \mathrm{C} \mathrm{NMR}(125 \mathrm{MHz}$, $\left.\mathrm{D}_{2} \mathrm{O}\right) \delta$ (ppm): 175.8, 75.5, 74.1, 62.6, 61.3, 57.9, 38.6, 21.6; HRMS (ESI): calcd for $\mathrm{C}_{8} \mathrm{H}_{17} \mathrm{O}_{4} \mathrm{~N}_{2}{ }^{+}\left[\mathrm{M}+\mathrm{H}^{+}\right]$ 205.1183, found 205.1180.

Data for 1- $\mathrm{N}$-acetylamino-2,5-imino-1,2,5-trideoxy-L-glucitol hydrochloride (F-10a): Colourless syrup, $44 \mathrm{mg}$, 99\% yield from compound F-5a (88 mg, $0.19 \mathrm{mmol}) ;[\alpha]_{\mathrm{D}}^{25}-29.2(c 1.05 \mathrm{in} \mathrm{MeOH})$; $v_{\max } / \mathrm{cm}^{-1}$ : 3285 (vs), 2931 (s), $1645(\mathrm{~m}), 1551$ (m), $1370(\mathrm{~m}), 1090(\mathrm{~m}), 1063(\mathrm{~m}) ;{ }^{1} \mathrm{H} \mathrm{NMR}(400 \mathrm{MHz}$, $\left.\mathrm{D}_{2} \mathrm{O}\right) \delta(\mathrm{ppm}): 4.30(\mathrm{t}, J=1.6 \mathrm{~Hz}, 1 \mathrm{H}), 4.16(\mathrm{t}, J=1.6 \mathrm{~Hz}, 1 \mathrm{H}), 4.01(\mathrm{dd}, J=8.8$ and $2.8 \mathrm{~Hz}, 1 \mathrm{H}), 3.95-3.88$ $(\mathrm{m}, 2 \mathrm{H}), 3.69-3.59(\mathrm{~m}, 3 \mathrm{H}), 2.04\left(\mathrm{~s}, 3 \mathrm{H}, \mathrm{COCH}_{3}\right) ;{ }^{13} \mathrm{C} \mathrm{NMR}\left(125 \mathrm{MHz}, \mathrm{D}_{2} \mathrm{O}\right) \delta(\mathrm{ppm}): 175.8,76.8,74.6$, 65.2, 63.1, 57.0, 39.4, 21.6; HRMS (ESI): calcd for $\mathrm{C}_{8} \mathrm{H}_{17} \mathrm{O}_{4} \mathrm{~N}_{2}{ }^{+}\left[\mathrm{M}+\mathrm{H}^{+}\right]$205.1183, found 205.1183. 
Data for 1- $\mathrm{N}$-acetylamino-2,5-imino-1,2,5-trideoxy-D-iditol hydrochloride (F-10b): Colourless syrup, $42 \mathrm{mg}$, 99\% yield from compound F-5b $(83 \mathrm{mg}, 0.17 \mathrm{mmol}) ;[\alpha]_{\mathrm{D}}{ }^{25}+7.7($ c $1.0 \mathrm{in} \mathrm{MeOH}) ; v_{\max } / \mathrm{cm}^{-1}$ : 3296 (vs), 2936 (s), 1651 (m), $1553(\mathrm{~m}), 1371(\mathrm{~m}), 1136$ (m), 1041 (m); ${ }^{1} \mathrm{H}$ NMR (500 MHz, $\left.\mathrm{D}_{2} \mathrm{O}\right) \delta(\mathrm{ppm})$ : $4.34(\mathrm{~d}, J=3.4 \mathrm{~Hz}, 1 \mathrm{H}), 4.27(\mathrm{t}, J=1.8 \mathrm{~Hz}, 1 \mathrm{H}), 4.00-3.94(\mathrm{~m}, 3 \mathrm{H}), 3.86(\mathrm{dd}, J=11.6$ and $8.3 \mathrm{~Hz}, 1 \mathrm{H}), 3.60$ $(\mathrm{d}, J=6.9 \mathrm{~Hz}, 2 \mathrm{H}), 2.01\left(\mathrm{~s}, 3 \mathrm{H}, \mathrm{COCH}_{3}\right) ;{ }^{13} \mathrm{C} \mathrm{NMR}\left(125 \mathrm{MHz}, \mathrm{D}_{2} \mathrm{O}\right) \delta(\mathrm{ppm}): 175.8,75.5,74.1,62.6,61.3$, 57.9, 38.6, 21.7; HRMS (ESI): calcd for $\mathrm{C}_{8} \mathrm{H}_{17} \mathrm{O}_{4} \mathrm{~N}_{2}{ }^{+}\left[\mathrm{M}+\mathrm{H}^{+}\right]$205.1183, found 205.1183.

Data for 1- $\mathrm{N}$-acetylamino-2,5-imino-1,2,5-trideoxy-D-allitol hydrochloride (G-10): Colourless syrup, $46 \mathrm{mg}, 99 \%$ yield from compound G-5 (92 mg, $0.19 \mathrm{mmol}) ;[\alpha]_{\mathrm{D}}^{23}-23.1($ c $1.0 \mathrm{in} \mathrm{MeOH}) ; v_{\max } / \mathrm{cm}^{-1}$ : 3306 (vs), 2928 (s), 1635 (s), 1551 (s), 1419 (m),1370 (m), 1089 (m), 1063 (m); ${ }^{1} \mathrm{H}$ NMR (500 MHz, $\left.\mathrm{D}_{2} \mathrm{O}\right)$ $\delta(\mathrm{ppm}): 4.22-4.19(\mathrm{~m}, 2 \mathrm{H}), 3.93(\mathrm{dd}, J=12.6 \mathrm{~Hz}$ and $3.8 \mathrm{~Hz}, 1 \mathrm{H}), 3.83(\mathrm{dd}, J=12.6 \mathrm{~Hz}$ and $3.8 \mathrm{~Hz}$, $1 \mathrm{H}), 3.78-3.74(\mathrm{~m}, 1 \mathrm{H}), 3.73-3.66(\mathrm{~m}, 2 \mathrm{H}), 3.57(\mathrm{dd}, J=15.1 \mathrm{~Hz}$ and $7.4 \mathrm{~Hz}, 1 \mathrm{H}), 2.04\left(\mathrm{~s}, 1 \mathrm{H}, \mathrm{COCH}_{3}\right)$; ${ }^{13} \mathrm{C}$ NMR (125 MHz, $\mathrm{D}_{2} \mathrm{O}$ ): $\delta$ (ppm): 176.1, 71.1, 70.2, 64.2, 62.8, 57.9, 38.5, 21.6; HRMS (ESI): calcd for $\mathrm{C}_{8} \mathrm{H}_{17} \mathrm{O}_{4} \mathrm{~N}_{2}{ }^{+}\left[\mathrm{M}+\mathrm{H}^{+}\right]$205.1183, found 205.1182.

Data for 1-N-acetylamino-2,5-imino-1,2,5-trideoxy-L-allitol hydrochloride (H-10): Colourless syrup, $41 \mathrm{mg}$, 99\% yield from compound $\mathbf{H}-5$ (81 mg, $0.17 \mathrm{mmol}) ;[\alpha]_{\mathrm{D}}^{23}+27.3(c 0.95 \mathrm{in} \mathrm{MeOH}) ; v_{\max } / \mathrm{cm}^{-1}$ : 3296 (vs), 2936 (s), 1651 (s), 1546 (s), 1419 (m), 1372 (m), 1091 (m), 1061 (m); ${ }^{1} \mathrm{H}$ NMR (500 MHz, D O) $\delta(\mathrm{ppm}): 4.22-4.18(\mathrm{~m}, 2 \mathrm{H}), 3.93(\mathrm{dd}, J=12.5 \mathrm{~Hz}$ and $3.6 \mathrm{~Hz}, 1 \mathrm{H}), 3.82(\mathrm{dd}, J=12.6 \mathrm{~Hz}$ and $3.6 \mathrm{~Hz}$, $1 \mathrm{H}), 3.78-3.74(\mathrm{~m}, 1 \mathrm{H}), 3.71-3.66(\mathrm{~m}, 2 \mathrm{H}), 3.57(\mathrm{dd}, J=15.1 \mathrm{~Hz}$ and $7.4 \mathrm{~Hz}, 1 \mathrm{H}), 2.04\left(\mathrm{~s}, 1 \mathrm{H}, \mathrm{COCH}_{3}\right)$; ${ }^{13} \mathrm{C}$ NMR (125 MHz, $\mathrm{D}_{2} \mathrm{O}$ ) $\delta(\mathrm{ppm})$ : 176.1, 71.1, 70.2, 64.2, 62.8, 57.9, 38.5, 21.5; HRMS (ESI): calcd for $\mathrm{C}_{8} \mathrm{H}_{17} \mathrm{O}_{4} \mathrm{~N}_{2}{ }^{+}\left[\mathrm{M}+\mathrm{H}^{+}\right]$205.1183, found 205.1184.

Data for 1-amino-2,5-imino-1,2,5-trideoxy-L-mannitol dihydrochloride (A-11) (Ref. [61], reported as free base): Colourless syrup, $46 \mathrm{mg}, 99 \%$ yield from compound A-3 $(86 \mathrm{mg}, 0.2 \mathrm{mmol}) ;[\alpha]_{\mathrm{D}}{ }^{23}-66.7(c$ 1.0 in $\mathrm{MeOH}) ; v_{\max } / \mathrm{cm}^{-1}: 3313$ (s), 2939 (s), $1115(\mathrm{~m}), 1058(\mathrm{~m}), 1033(\mathrm{~m}), 1014(\mathrm{~m}) ;{ }^{1} \mathrm{H}$ NMR $(400 \mathrm{MHz}$, $\left.\mathrm{D}_{2} \mathrm{O}\right) \delta(\mathrm{ppm}): 4.20-4.12(\mathrm{~m}, 2 \mathrm{H}), 3.98(\mathrm{dd}, J=12.5 \mathrm{~Hz}$ and $3.5 \mathrm{~Hz}, 1 \mathrm{H}), 3.91-3.81(\mathrm{~m}, 2 \mathrm{H}), 3.74-3.69$ $(\mathrm{m}, 1 \mathrm{H}), 3.57(\mathrm{~d}, J=10.5 \mathrm{~Hz}, 2 \mathrm{H}) ;{ }^{13} \mathrm{C} \mathrm{NMR}\left(125 \mathrm{MHz}, \mathrm{D}_{2} \mathrm{O}\right) \delta(\mathrm{ppm}): 76.5,73.9,63.4,58.4,58.1,38.7$; HRMS (ESI): calcd for $\mathrm{C}_{6} \mathrm{H}_{15} \mathrm{O}_{3} \mathrm{~N}_{2}{ }^{+}\left[\mathrm{M}+\mathrm{H}^{+}\right]$163.1077, found 163.1077 .

Data for 1-amino-2,5-imino-1,2,5-trideoxy-D-mannitol dihydrochloride (B-11) (Ref. [17]): Colourless syrup, $35 \mathrm{mg}$, 99\% yield from compound B-3 $(66 \mathrm{mg}, 0.15 \mathrm{mmol}) ;[\alpha]_{\mathrm{D}}^{23}-66.7$ (c 1.0 in $\left.\mathrm{MeOH}\right)$; $v_{\text {max }} / \mathrm{cm}^{-1}$ : $3314(\mathrm{~s}), 2942(\mathrm{~s}), 1113(\mathrm{~m}), 1063(\mathrm{~m}), 1033(\mathrm{~m}), 1014(\mathrm{~m}) ;{ }^{1} \mathrm{H}$ NMR (400 MHz, $\left.\mathrm{D}_{2} \mathrm{O}\right) \delta(\mathrm{ppm})$ : $4.19-4.12(\mathrm{~m}, 2 \mathrm{H}), 3.98(\mathrm{dd}, J=12.6 \mathrm{~Hz}$ and $3.6 \mathrm{~Hz}, 1 \mathrm{H}), 3.88(\mathrm{dd}, J=12.5 \mathrm{~Hz}$ and $3.6 \mathrm{~Hz}, 1 \mathrm{H}), 3.83(\mathrm{q}, J$ $=7.2 \mathrm{~Hz}, 1 \mathrm{H}), 3.73-3.69(\mathrm{~m}, 1 \mathrm{H}), 3.56(\mathrm{~d}, J=10.5 \mathrm{~Hz}, 2 \mathrm{H}) ;{ }^{13} \mathrm{C} \mathrm{NMR}\left(125 \mathrm{MHz}, \mathrm{D}_{2} \mathrm{O}\right) \delta(\mathrm{ppm}): 76.5$, 73.9, 63.4, 58.4, 58.1, 38.7; HRMS (ESI): calcd for $\mathrm{C}_{6} \mathrm{H}_{15} \mathrm{O}_{3} \mathrm{~N}_{2}{ }^{+}\left[\mathrm{M}+\mathrm{H}^{+}\right]$163.1077, found 163.1077.

Data for 1-amino-2,5-imino-1,2,5-trideoxy-L-altritol dihydrochloride (C-11) (Ref. [17]): Colourless syrup, $39 \mathrm{mg}$, 99\% yield from compound C-3 (73 mg, $0.17 \mathrm{mmol}) ;[\alpha]_{\mathrm{D}}^{23}-42.0($ c 1.0 in $\mathrm{MeOH})$; $v_{\text {max }} / \mathrm{cm}^{-1}: 3314(\mathrm{~s}), 2923(\mathrm{~s}), 1132(\mathrm{~m}), 1033(\mathrm{~m}), 1014(\mathrm{~m}) ;{ }^{1} \mathrm{H}$ NMR $\left(500 \mathrm{MHz}, \mathrm{D}_{2} \mathrm{O}\right) \delta(\mathrm{ppm}): 4.38(\mathrm{t}$, $J=3.2 \mathrm{~Hz}, 1 \mathrm{H}), 4.34(\mathrm{dd}, J=9.5 \mathrm{~Hz}$ and $3.7 \mathrm{~Hz}, 1 \mathrm{H}), 4.04-3.97(\mathrm{~m}, 1 \mathrm{H}), 3.97-3.92(\mathrm{~m}, 2 \mathrm{H}), 3.85(\mathrm{ddd}$, $J=13.8 \mathrm{~Hz}$ and $7.8 \mathrm{~Hz}$ and $5.9 \mathrm{~Hz}, 1 \mathrm{H}), 3.62-3.53(\mathrm{~m}, 2 \mathrm{H}) ;{ }^{13} \mathrm{C} \mathrm{NMR}\left(125 \mathrm{MHz}, \mathrm{D}_{2} \mathrm{O}\right) \delta(\mathrm{ppm}): 74.0$, 69.3, 62.9, 57.3, 56.9, 39.0; HRMS (ESI): calcd for $\mathrm{C}_{6} \mathrm{H}_{15} \mathrm{O}_{3} \mathrm{~N}_{2}{ }^{+}\left[\mathrm{M}+\mathrm{H}^{+}\right]$163.1077, found 163.1078.

Data for 1-amino-2,5-imino-1,2,5-trideoxy-D-altritol dihydrochloride (D-11) (Ref. [61], reported as free base): Colourless syrup, $37 \mathrm{mg}$, 99\% yield from compound D-3 $(69 \mathrm{mg}, 0.2 \mathrm{mmol}) ;[\alpha]_{\mathrm{D}}{ }^{23}+39.0(\mathrm{c}$ 0.8 in $\mathrm{MeOH}) ; v_{\max } / \mathrm{cm}^{-1}: 3318(\mathrm{~s}), 2923(\mathrm{~s}), 1131(\mathrm{~m}), 1033(\mathrm{~m}), 983(\mathrm{~m}) ;{ }^{1} \mathrm{H}$ NMR $\left(500 \mathrm{MHz}, \mathrm{D}_{2} \mathrm{O}\right)$ $\delta(\mathrm{ppm}): 4.38(\mathrm{t}, J=2.5 \mathrm{~Hz}, 1 \mathrm{H}), 4.34(\mathrm{dd}, J=9.5 \mathrm{~Hz}$ and $3.7 \mathrm{~Hz}, 1 \mathrm{H}), 4.06-4.01(\mathrm{~m}, 1 \mathrm{H}), 3.98-3.93(\mathrm{~m}$, $2 \mathrm{H}), 3.86(\mathrm{dd}, J=14.9 \mathrm{~Hz}$ and $7.9 \mathrm{~Hz}, 1 \mathrm{H}), 3.62-3.53(\mathrm{~m}, 2 \mathrm{H}) ;{ }^{13} \mathrm{C} \mathrm{NMR}\left(125 \mathrm{MHz}, \mathrm{D}_{2} \mathrm{O}\right) \delta(\mathrm{ppm}): 74.0$, 69.3, 62.9, 57.3, 56.9, 39.0; HRMS (ESI): calcd for $\mathrm{C}_{6} \mathrm{H}_{15} \mathrm{O}_{3} \mathrm{~N}_{2}{ }^{+}\left[\mathrm{M}+\mathrm{H}^{+}\right]$163.1077, found 163.1076 .

Data for 1-amino-2,5-imino-1,2,5-trideoxy-D-glucitol dihydrochloride (E-11a) (Ref. [17]): Colourless syrup, $38 \mathrm{mg}$, 99\% yield from compound E-3a $(71 \mathrm{mg}, 0.16 \mathrm{mmol}) ;[\alpha]_{\mathrm{D}}{ }^{23}+21.6(c 0.5 \mathrm{in} \mathrm{MeOH})$; $v_{\text {max }} / \mathrm{cm}^{-1}$ : 3313 (s), 2935 (s), $1118(\mathrm{~m}), 1063(\mathrm{~m}), 1033$ (m); ${ }^{1} \mathrm{H}$ NMR (300 MHz, $\left.\mathrm{D}_{2} \mathrm{O}\right) \delta(\mathrm{ppm}): 4.32-4.29$ 
$(\mathrm{m}, 1 \mathrm{H}), 4.26(\mathrm{t}, J=2.8 \mathrm{~Hz}, 1 \mathrm{H}), 4.02-3.90(\mathrm{~m}, 3 \mathrm{H}), 3.80(\mathrm{dt}, J=6.9$ and $2.9 \mathrm{~Hz}, 1 \mathrm{H}), 3.54(\mathrm{~d}, J=6.8 \mathrm{~Hz}, 2 \mathrm{H})$; ${ }^{13} \mathrm{C}$ NMR $\left(125 \mathrm{MHz}, \mathrm{D}_{2} \mathrm{O}\right) \delta$ (ppm): 77.5, 74.1, 64.1, 62.4, 56.9, 39.2; HRMS (ESI): calcd for $\mathrm{C}_{6} \mathrm{H}_{15} \mathrm{O}_{3} \mathrm{~N}_{2}{ }^{+}$ $\left[\mathrm{M}+\mathrm{H}^{+}\right]$163.1077, found 163.1077 .

Data for 1-amino-2,5-imino-1,2,5-trideoxy-L-iditol dihydrochloride (E-11b) (Ref. [17]): Colourless syrup, $44 \mathrm{mg}, 99 \%$ yield from compound E-3b $(81 \mathrm{mg}, 0.19 \mathrm{mmol}) ;[\alpha]_{\mathrm{D}}^{23}+5.5($ c 1.0 in $\mathrm{MeOH})$; $v_{\max } / \mathrm{cm}^{-1}: 3322(\mathrm{~s}), 2924(\mathrm{~s}), 1131(\mathrm{~m}), 1039(\mathrm{~m}), 984(\mathrm{~m}) ;{ }^{1} \mathrm{H}$ NMR $\left(500 \mathrm{MHz}, \mathrm{D}_{2} \mathrm{O}\right) \delta(\mathrm{ppm}): 4.46(\mathrm{~d}$, $J=5.1 \mathrm{~Hz}, 1 \mathrm{H}), 4.44(\mathrm{~d}, J=5.1 \mathrm{~Hz}, 1 \mathrm{H}), 4.22(\mathrm{dt}, J=6.8 \mathrm{~Hz}$ and $3.5 \mathrm{~Hz}, 1 \mathrm{H}), 4.12-4.09(\mathrm{~m}, 1 \mathrm{H}), 4.03(\mathrm{dd}$, $J=12.2 \mathrm{~Hz}$ and $4.6 \mathrm{~Hz}, 1 \mathrm{H}), 3.94(\mathrm{dd}, J=12.2 \mathrm{~Hz}$ and $8.8 \mathrm{~Hz}, 1 \mathrm{H}), 3.62(\mathrm{dd}, J=13.7 \mathrm{~Hz}$ and $6.8 \mathrm{~Hz}, 1 \mathrm{H})$, 3.55-3.51(dd, $J=13.7 \mathrm{~Hz}$ and $6.8 \mathrm{~Hz}, 1 \mathrm{H}) ;{ }^{13} \mathrm{C} \mathrm{NMR}\left(125 \mathrm{MHz}, \mathrm{D}_{2} \mathrm{O}\right) \delta(\mathrm{ppm}): 74.6,74.2,63.8,58.3,57.3$, 35.9; HRMS (ESI): calcd for $\mathrm{C}_{6} \mathrm{H}_{15} \mathrm{O}_{3} \mathrm{~N}_{2}{ }^{+}\left[\mathrm{M}+\mathrm{H}^{+}\right]$163.1077, found 163.1076 .

Data for 1-amino-2,5-imino-1,2,5-trideoxy-L-glucitol dihydrochloride (F-11a) (Ref. [61], reported as free base): Colourless syrup, $31 \mathrm{mg}$, 99\% yield from compound F-3a (57 mg, $0.13 \mathrm{mmol}) ;[\alpha]_{\mathrm{D}}{ }^{23}-27.5$ (c 0.65 in MeOH); $v_{\max } / \mathrm{cm}^{-1}: 3313$ (s), 2935 (s), 1118 (m), 1063 (m), 1033 (m); ${ }^{1} \mathrm{H}$ NMR (500 MHz, $\left.\mathrm{D}_{2} \mathrm{O}\right)$ $\delta$ (ppm): $4.35(\mathrm{~s}, 1 \mathrm{H}), 4.31(\mathrm{~s}, 1 \mathrm{H}), 4.05-3.96(\mathrm{~m}, 3 \mathrm{H}), 3.88-3.84(\mathrm{~m}, 1 \mathrm{H}), 3.59(\mathrm{~d}, J=6.6 \mathrm{~Hz}, 2 \mathrm{H}) ;{ }^{13} \mathrm{C}$ NMR (125 MHz, $\left.\mathrm{D}_{2} \mathrm{O}\right) \delta(\mathrm{ppm})$ : 77.4, 74.0, 64.1, 62.5, 56.8, 39.2; HRMS (ESI): calcd for $\mathrm{C}_{6} \mathrm{H}_{15} \mathrm{O}_{3} \mathrm{~N}_{2}{ }^{+}$ $\left[\mathrm{M}+\mathrm{H}^{+}\right]$163.1077, found 163.1076.

Data for 1-amino-2,5-imino-1,2,5-trideoxy-D-iditol dihydrochloride (F-11b) (Ref. [61], reported as free base): Colourless syrup, $34 \mathrm{mg}, 99 \%$ yield from compound $\mathbf{F}-3 \mathbf{b}(62 \mathrm{mg}, 0.14 \mathrm{mmol}) ;[\alpha]_{\mathrm{D}}{ }^{23}-7.3$ (c 0.3 in $\mathrm{MeOH}) ; v_{\max } / \mathrm{cm}^{-1}: 3310$ (s), $2936(\mathrm{~s}), 1131(\mathrm{~m}), 1076(\mathrm{~m}), 1031(\mathrm{~m}) ;{ }^{1} \mathrm{H}$ NMR $\left(500 \mathrm{MHz}, \mathrm{D}_{2} \mathrm{O}\right) \delta$ (ppm): $4.41-4.39(\mathrm{~m}, 2 \mathrm{H}), 4.22(\mathrm{dt}, J=6.7 \mathrm{~Hz}$ and $3.5 \mathrm{~Hz}, 1 \mathrm{H}), 4.05-3.99(\mathrm{~m}, 2 \mathrm{H}), 3.91(\mathrm{dd}, J=11.8 \mathrm{~Hz}$ and $8.5 \mathrm{~Hz}, 1 \mathrm{H}), 3.57(\mathrm{dd}, J=13.7 \mathrm{~Hz}$ and $6.8 \mathrm{~Hz}, 1 \mathrm{H}), 3.47(\mathrm{dd}, J=13.7 \mathrm{~Hz}$ and $6.8 \mathrm{~Hz}, 1 \mathrm{H}) ;{ }^{13} \mathrm{C} \mathrm{NMR}$ (125 MHz, $\mathrm{D}_{2} \mathrm{O}$ ) $\delta$ (ppm): 74.7, 74.4, 63.6, 58.1, 57.4, 36.0; HRMS (ESI): calcd for $\mathrm{C}_{6} \mathrm{H}_{15} \mathrm{O}_{3} \mathrm{~N}_{2}{ }^{+}\left[\mathrm{M}+\mathrm{H}^{+}\right]$ 163.1077, found 163.1076 .

Data for 1-amino-2,5-imino-1,2,5-trideoxy-D-allitol dihydrochloride (G-11) (Ref. [61], reported as free base): Colourless syrup, $35 \mathrm{mg}$, 99\% yield from compound G-3 $(65 \mathrm{mg}, 0.15 \mathrm{mmol}) ;[\alpha]_{\mathrm{D}}{ }^{23}+8.1($ c 1.0 in $\mathrm{MeOH}) ; v_{\max } / \mathrm{cm}^{-1}: 3322(\mathrm{~s}), 2947(\mathrm{~s}), 1122(\mathrm{~m}), 1078(\mathrm{~m}), 1034(\mathrm{~m}), 979(\mathrm{~m}) ;{ }^{1} \mathrm{H}$ NMR $(500 \mathrm{MHz}$, $\left.\mathrm{D}_{2} \mathrm{O}\right) \delta(\mathrm{ppm}): 4.33(\mathrm{dd}, J=7.6 \mathrm{~Hz}$ and $5.0 \mathrm{~Hz}, 1 \mathrm{H}), 4.29(\mathrm{dd}, J=4.7 \mathrm{~Hz}$ and $4.0 \mathrm{~Hz}, 1 \mathrm{H}), 3.96(\mathrm{dd}$, $J=12.2 \mathrm{~Hz}$ and $3.5 \mathrm{~Hz} 1 \mathrm{H}), 3.92-3.84(\mathrm{~m}, 3 \mathrm{H}) 3.61-3.53(\mathrm{~m}, 2 \mathrm{H}) ;{ }^{13} \mathrm{C} \mathrm{NMR}\left(125 \mathrm{MHz}, \mathrm{D}_{2} \mathrm{O}\right) \delta(\mathrm{ppm})$ : 72.5, 70.0, 66.0, 58.6, 58.1, 38.4; HRMS (ESI): calcd for $\mathrm{C}_{6} \mathrm{H}_{15} \mathrm{O}_{3} \mathrm{~N}_{2}{ }^{+}\left[\mathrm{M}+\mathrm{H}^{+}\right]$163.1077, found 163.1077.

Data for 1-amino-2,5-imino-1,2,5-trideoxy-L-allitol dihydrochloride (H-11) (Ref. [61], reported as free base): Colourless syrup, $36 \mathrm{mg}$, 99\% yield from compound $\mathbf{H}-3(67 \mathrm{mg}, 0.16 \mathrm{mmol}) ;[\alpha]_{\mathrm{D}}{ }^{22}-7.2$ (c 1.0 in $\mathrm{MeOH}) ; v_{\max } / \mathrm{cm}^{-1}: 3313(\mathrm{~s}), 2951(\mathrm{~s}), 1124(\mathrm{~m}), 1079(\mathrm{~m}), 1034(\mathrm{~m}), 980(\mathrm{~m}) ;{ }^{1} \mathrm{H}$ NMR $(400 \mathrm{MHz}$, $\left.\mathrm{D}_{2} \mathrm{O}\right) \delta(\mathrm{ppm}): 4.34-4.27(\mathrm{~m}, 2 \mathrm{H}), 3.96(\mathrm{dd}, J=12.0 \mathrm{~Hz}$ and $3.4 \mathrm{~Hz}, 1 \mathrm{H}), 3.92-3.84(\mathrm{~m}, 3 \mathrm{H}), 3.62-3.52$ (m, 2H); ${ }^{13} \mathrm{C}$ NMR (125 MHz, $\left.\mathrm{D}_{2} \mathrm{O}\right) \delta$ (ppm): 72.5, 70.1, 66.1, 58.7, 58.1, 38.4; HRMS (ESI): calcd for $\mathrm{C}_{6} \mathrm{H}_{15} \mathrm{O}_{3} \mathrm{~N}_{2}{ }^{+}\left[\mathrm{M}+\mathrm{H}^{+}\right]$163.1077, found 163.1077.

\section{Conclusions}

In summary, a general and efficient synthetic strategy has been developed for the synthesis of 1-N-acetylamino and 1-amino pyrrolidine analogues of pochonicine (1) with L-arabino-nitrone (A), D-arabino-nitrone (B), L-lyxo-nitrone (C), D-lyxo-nitrone (D), L-xylo-nitrone (E), D-xylo-nitrone (F), L-ribo-nitrone $(\mathbf{G})$ and D-ribo-nitrone $(\mathbf{H})$ as the starting materials 4 and 5 steps, respectively. Glycosidase inhibition assays on a range of enzymes showed that $1-N$-acetylamino pyrrolidine analogues with the same configuration as DMDP and pochonicine (1) showed powerful inhibition of $\beta$-HexNAcases and moderate inhibition of $\alpha$-GalNAcase, while the other compounds showed weak or no inhibition of the tested glycosidases. This work has further examined the glycosidase inhibition of pyrrolidine analogues of pochonicine and its stereoisomers, and would be helpful for the study of potent and selective $\beta$-HexNAcase inhibitors. 
Supplementary Materials: The following are available online at http://www.mdpi.com/1420-3049/25/7/1498/s1: Copies of ${ }^{1} \mathrm{H}$ NMR, ${ }^{13} \mathrm{C}$ NMR and 2D NMR spectra, and data of crystal structures.

Author Contributions: X.Y. performed the chemical syntheses, analysed the data and wrote the original draft; Y.S. and A.K. performed the glycosidase inhibition assay; Y.-X.L. and Y.-M.J. validated all the experimental data and revised the manuscript; A.K., G.W.J.F. and C.-Y.Y. designed the experiments and reviewed the manuscript. All authors approved the final manuscript. All authors have read and agreed to the published version of the manuscript.

Funding: Financial support from the National Natural Science Foundation of China (No. 21772206) and National Engineering Research Center for Carbohydrate Synthesis of Jiangxi Normal University is gratefully acknowledged. This work was supported in part by a Grant-in-Aid for Scientific Research (C) from the Japanese Society for the Promotion of Science (JSPS KAKENHI Grant Number JP17K08362) (AK).

Conflicts of Interest: The authors declare no conflict of interest.

\section{References}

1. Usuki, H.; Toyo-oka, M.; Kanzaki, H.; Okuda, T.; Nitoda, T. Pochonicine, a polyhydroxylated pyrrolizidine alkaloid from fungus Pochonia suchlasporia var. suchlasporia TAMA 87 as a potent $\beta-N-$ acetylglucosaminidase inhibitor. Bioorg. Med. Chem. 2009, 17, 7248-7253. [CrossRef] [PubMed]

2. Zhu, J.-S.; Nakagawa, S.; Chen, W.; Adachi, I.; Jia, Y.-M.; Hu, X.-G.; Fleet, G.W.J.; Wilson, F.X.; Nitoda, T.; Horne, G.; et al. Synthesis of Eight Stereoisomers of Pochonicine: Nanomolar Inhibition of $\beta-N-$ Acetylhexosaminidases. J. Org. Chem. 2013, 78, 10298-10309. [CrossRef] [PubMed]

3. Stuetz, A.E.; Wrodnigg, T.M. Carbohydrate-Processing Enzymes of the Lysosome: Diseases Caused by Misfolded Mutants and Sugar Mimetics as Correcting Pharmacological Chaperones. In Advances in Carbohydrate Chemistry and Biochemistry; Baker, D.C., Ed.; Elsevier: Amsterdam, The Netherlands, 2016; Volume 73, pp. 225-302.

4. Rast, D.M.; Baumgartner, D.; Mayer, C.; Hollenstein, G.O. Cell wall-associated enzymes in fungi. Phytochemistry 2003, 64, 339-366. [CrossRef]

5. Merzendorfer, H.; Zimoch, L. Chitin metabolism in insects: Structure, function and regulation of chitin synthases and chitinases. J. Exp. Biol. 2003, 206, 4393-4412. [CrossRef]

6. Hart, G.W.; Housley, M.P.; Slawson, C. Cycling of O-linked $\beta$-N-acetylglucosamine on nucleocytoplasmic proteins. Nature 2007, 446, 1017-1022. [CrossRef]

7. Boyd, R.E.; Lee, G.; Rybczynski, P.; Benjamin, E.R.; Khanna, R.; Wustman, B.A.; Valenzano, K.J. Pharmacological Chaperones as Therapeutics for Lysosomal Storage Diseases. J. Med. Chem. 2013, 56, 2705-2725. [CrossRef]

8. Vosseller, K.; Wells, L.; Lane, M.D.; Hart, G.W. Elevated nucleocytoplasmic glycosylation by O-GlcNAc results in insulin resistance associated with defects in Akt activation in 3T3-L1 adipocytes. Proc. Nat. Acad. Sci. USA 2002, 99, 5313-5318. [CrossRef]

9. Yuzwa, S.A.; Macauley, M.S.; Heinonen, J.E.; Shan, X.; Dennis, R.J.; He, Y.; Whitworth, G.E.; Stubbs, K.A.; McEachern, E.J.; Davies, G.J.; et al. A potent mechanism-inspired O-GlcNAcase inhibitor that blocks phosphorylation of tau in vivo. Nat. Chem. Biol. 2008, 4, 483-490. [CrossRef]

10. Scott, A.Y.; David, J.V. O-GlcNAc Modification and the Tauopathies: Insights from Chemical Biology. Curr. Alzheimer Res. 2009, 6, 451-454.

11. Cecioni, S.; Vocadlo, D.J. Tools for probing and perturbing O-GlcNAc in cells and in vivo. Curr. Opin. Chem. Biol. 2013, 17, 719-728. [CrossRef]

12. Aoyama, T.; Naganawa, H.; Suda, H.; Uotani, K.; Aoyagi, T.; Takeuchi, T. The structure of nagstatin, a new inhibitor of $N$-acetyl- $\beta$-D-glucosaminidase. J. Antibiot. 1992, 45, 1557-1558. [CrossRef] [PubMed]

13. Umezawa, H.; Aoyagi, T.; Komiyama, T.; Morishima, H.; Hamada, M.; Takeuchi, T. Purification and characterization of a sialidase inhibitor, siastatin, produced by streptomyces. J. Antibiot. 1974, 27, 963-969. [CrossRef] [PubMed]

14. Tatsuta, K.; Miura, S.; Ohta, S.; Gunji, H. Syntheses and glycosidase inhibiting activities of nagstatin analogs. J. Antibiot. 1995, 48, 286-288. [CrossRef] [PubMed]

15. Liang, P.H.; Cheng, W.C.; Lee, Y.L.; Yu, H.P.; Wu, Y.T.; Lin, Y.L.; Wong, C.H. Novel five-membered iminocyclitol derivatives as selective and potent glycosidase inhibitors: New structures for antivirals and osteoarthritis. Chembiochem 2006, 7, 165-173. [CrossRef] 
16. Tsou, E.-L.; Yeh, Y.-T.; Liang, P.-H.; Cheng, W.-C. A convenient approach toward the synthesis of enantiopure isomers of DMDP and ADMDP. Tetrahedron 2009, 65, 93-100. [CrossRef]

17. Win-Mason, A.L.; Jongkees, S.A.K.; Withers, S.G.; Tyler, P.C.; Timmer, M.S.M.; Stocker, B.L. Stereoselective Total Synthesis of Aminoiminohexitols via Carbamate Annulation. J. Org. Chem. 2011, 76, 9611-9621. [CrossRef]

18. Ayers, B.J.; Glawar, A.F.G.; Martínez, R.F.; Ngo, N.; Liu, Z.; Fleet, G.W.J.; Butters, T.D.; Nash, R.J.; Yu, C.-Y.; Wormald, M.R.; et al. Nine of 16 Stereoisomeric Polyhydroxylated Proline Amides Are Potent $\beta-N$-Acetylhexosaminidase Inhibitors. J. Org. Chem. 2014, 79, 3398-3409. [CrossRef]

19. Glawar, A.F.G.; Martinez, R.F.; Ayers, B.J.; Hollas, M.A.; Ngo, N.; Nakagawa, S.; Kato, A.; Butters, T.D.; Fleet, G.W.J.; Jenkinson, S.F. Structural essentials for $\beta$-N-acetylhexosaminidase inhibition by amides of prolines, pipecolic and azetidine carboxylic acids. Org. Biomol. Chem. 2016, 14, 10371-10385. [CrossRef]

20. Rountree, J.S.S.; Butters, T.D.; Wormald, M.R.; Dwek, R.A.; Asano, N.; Ikeda, K.; Evinson, E.L.; Nash, R.J.; Fleet, G.W.J. Efficient synthesis from D-lyxonolactone of 2-acetamido-1,4-imino-1,2,4-trideoxy-L-arabinitol LABNAc, a potent pyrrolidine inhibitor of hexosaminidases. Tetrahedron Lett. 2007, 48, 4287-4291. [CrossRef]

21. Crabtree, E.V.; Martinez, R.F.; Nakagawa, S.; Adachi, I.; Butters, T.D.; Kato, A.; Fleet, G.W.J.; Glawar, A.F.G. Synthesis of the enantiomers of XYLNAc and LYXNAc: Comparison of $\beta-N$-acetylhexosaminidase inhibition by the 8 stereoisomers of 2-N-acetylamino-1,2,4-trideoxy-1,4-iminopentitols. Org. Biomol. Chem. 2014, 12, 3932-3943. [CrossRef]

22. Tran, A.T.; Luo, B.; Jagadeesh, Y.; Auberger, N.; Désiré, J.; Nakagawa, S.; Kato, A.; Zhang, Y.; Blériot, Y.; Sollogoub, M. Synthesis of pyrrolidine-based analogues of 2-acetamidosugars as $N$-acetyl-D-glucosaminidase inhibitors. Carbohydr. Res. 2015, 409, 56-62. [CrossRef]

23. Fleet, G.W.J.; Smith, P.W.; Nash, R.J.; Fellows, L.E.; Parekh, R.B.; Rademacher, T.W. Synthesis of 2-Acetamido-1,5-imino-1,2,5-trideoxy-D-mannitol and of 2-Acetamido-1,5-imino-1,2,5-trideoxy-D-glucitol, a Potent and Specific Inhibitor of a Number of $\beta$-N-Acetylglucosaminidases. Chem. Lett. 1986, 15, 1051-1054. [CrossRef]

24. Glawar, A.F.G.; Best, D.; Ayers, B.J.; Miyauchi, S.; Nakagawa, S.; Aguilar-Moncayo, M.; García Fernández, J.M.; Ortiz Mellet, C.; Crabtree, E.V.; Butters, T.D.; et al. Scalable Syntheses of Both Enantiomers of DNJNAc and DGJNAc from Glucuronolactone: The Effect of $N$-Alkylation on Hexosaminidase Inhibition. Chem. Eur. J. 2012, 18, 9341-9359. [CrossRef] [PubMed]

25. de la Fuente, A.; Verdaguer, X.; Riera, A. Stereodivergent Syntheses of altro and manno Stereoisomers of 2-Acetamido-1,2-dideoxynojirimycin. Eur. J. Org. Chem. 2017, 47, 7179-7185. [CrossRef]

26. Blériot, Y.; Tran, A.T.; Prencipe, G.; Jagadeesh, Y.; Auberger, N.; Zhu, S.; Gauthier, C.; Zhang, Y.; Désiré, J.; Adachi, I.; et al. Synthesis of 1,2-trans-2-Acetamido-2-deoxyhomoiminosugars. Org. Lett. 2014, 16, 5516-5519. [CrossRef]

27. Li, H.; Marcelo, F.; Bello, C.; Vogel, P.; Butters, T.D.; Rauter, A.P.; Zhang, Y.; Sollogoub, M.; Blériot, Y. Design and synthesis of acetamido tri- and tetra-hydroxyazepanes: Potent and selective $\beta$ - $N$-acetylhexosaminidase inhibitors. Bioorg. Med. Chem. 2009, 17, 5598-5604. [CrossRef]

28. Marcelo, F.; He, Y.; Yuzwa, S.A.; Nieto, L.; Jiménez-Barbero, J.; Sollogoub, M.; Vocadlo, D.J.; Davies, G.D.; Blériot, Y. Molecular Basis for Inhibition of GH84 Glycoside Hydrolases by Substituted Azepanes: Conformational Flexibility Enables Probing of Substrate Distortion. J. Am. Chem. Soc. 2009, 131, 5390-5392. [CrossRef]

29. Mondon, M.; Hur, S.; Vadlamani, G.; Rodrigues, P.; Tsybina, P.; Oliver, A.; Mark, B.L.; Vocadlo, D.J.; Blériot, Y. Selective trihydroxyazepane NagZ inhibitors increase sensitivity of Pseudomonas aeruginosa to $\beta$-lactams. Chem. Commun. 2013, 49, 10983-10985. [CrossRef]

30. Glawar, A.F.G.; Jenkinson, S.F.; Thompson, A.L.; Nakagawa, S.; Kato, A.; Butters, T.D.; Fleet, G.W.J. 3-Hydroxyazetidine Carboxylic Acids: Non-Proteinogenic Amino Acids for Medicinal Chemists. Chemmedchem 2013, 8, 658-666. [CrossRef]

31. Liu, Z.; Jenkinson, S.F.; Kato, A.; Nakagawa, S.; Wormald, M.R.; Yu, C.-Y.; Fleet, G.W.J. 3-Azidoazetidines as the first scaffolds for $\beta$-amino azetidine carboxylic acid peptidomimetics: Azetidine iminosugars containing an acetamido group do not inhibit $\beta-\mathrm{N}$-acetylhexosaminidases. Tetrahedron Asymmetry 2016, 27, 872-881. [CrossRef]

32. Harit, V.K.; Ramesh, N.G. Amino-functionalized iminocyclitols: Synthetic glycomimetics of medicinal interest. Rsc Advances 2016, 6, 109528-109607. [CrossRef] 
33. Kitamura, Y.; Koshino, H.; Nakamura, T.; Tsuchida, A.; Nitoda, T.; Kanzaki, H.; Matsuoka, K.; Takahashi, S. Total synthesis of the proposed structure for pochonicine and determination of its absolute configuration. Tetrahedron Lett. 2013, 54, 1456-1459. [CrossRef]

34. Salunke, R.V.; Ramesh, N.G. A Concise Total Synthesis of the Stereoisomers of (-)-Pochonicine. Eur. J. Org. Chem. 2016, 654-657. [CrossRef]

35. Martinez, S.T.; Belouezzane, C.; Pinto, A.C.; Glasnov, T. Synthetic Strategies towards the Azabicyclo 3.3.0 -Octane Core of Natural Pyrrolizidine Alkaloids. An Overview. Org. Prep. Proced. Int. 2016, 48, 223-253. [CrossRef]

36. Robertson, J.; Stevens, K. Pyrrolizidine alkaloids: Occurrence, biology, and chemical synthesis. Nat. Prod. Rep. 2017, 34, 62-89. [CrossRef] [PubMed]

37. O'Keefe, S.; Roebuck, Q.P.; Nakagome, I.; Hirono, S.; Kato, A.; Nash, R.; High, S. Characterizing the selectivity of ER $\alpha$-glucosidase inhibitors. Glycobiology 2019, 29, 530-542. [CrossRef]

38. Ferhati, X.; Matassini, C.; Fabbrini, M.G.; Goti, A.; Morrone, A.; Cardona, F.; Moreno-Vargas, A.J.; Paoli, P. Dual targeting of PTP1B and glucosidases with new bifunctional iminosugar inhibitors to address type 2 diabetes. Bioorg. Chem. 2019, 87, 534-549. [CrossRef]

39. Yang, L.-F.; Shimadate, Y.; Kato, A.; Li, Y.-X.; Jia, Y.-M.; Fleet, G.W.J.; Yu, C.-Y. Synthesis and glycosidase inhibition of N-substituted derivatives of DIM. Org. Biomol. Chem. 2020, 18, 999-1011. [CrossRef]

40. Suzuki, K.; Nakahara, T.; Kanie, O. 3,4-dihydroxypyrrolidine as glycosidase inhibitor. Curr. Top. Med. Chem. 2009, 9, 34-57. [CrossRef]

41. Takaoka, Y.; Kajimoto, T.; Wong, C.H. Inhibition of $N$-acetylglucosaminyl transfer enzymes: Chemicalenzymic synthesis of new five-membered acetamido azasugars. J. Org. Chem. 1993, 58, 4809-4812. [CrossRef]

42. Liu, J.J.; Numa, M.M.D.; Liu, H.T.; Huang, S.J.; Sears, P.; Shikhman, A.R.; Wong, C.H. Synthesis and HighThroughput Screening of $N$-Acetyl- $\beta$-hexosaminidase Inhibitor Libraries Targeting Osteoarthritis. J. Org. Chem. 2004, 69, 6273-6283. [CrossRef] [PubMed]

43. Li, Y.-X.; Kinami, K.; Hirokami, Y.; Kato, A.; Su, J.-K.; Jia, Y.-M.; Fleet, G.W.J.; Yu, C.-Y. Gem-difluoromethylated and trifluoromethylated derivatives of DMDP-related iminosugars: Synthesis and glycosidase inhibition. Org. Biomol. Chem. 2016, 14, 2249-2263. [CrossRef] [PubMed]

44. Song, Y.-Y.; Kinami, K.; Kato, A.; Jia, Y.-M.; Li, Y.-X.; Fleet, G.W.J.; Yu, C.-Y. First total synthesis of (+)-broussonetine W: Glycosidase inhibition of natural product \& analogs. Org. Biomol. Chem. 2016, 14, 5157-5174. [PubMed]

45. Cheng, B.; Hirokami, Y.; Li, Y.-X.; Kato, A.; Jia, Y.-M.; Yu, C.-Y. Synthesis and glycosidase inhibition of C-7 modified casuarine derivatives. Chin. Chem. Lett. 2017, 28, 1701-1704. [CrossRef]

46. Wu, Q.-K.; Kinami, K.; Kato, A.; Li, Y.-X.; Jia, Y.-M.; Fleet, G.W.J.; Yu, C.-Y. Synthesis and Glycosidase Inhibition of Broussonetine M and Its Analogues. Molecules 2019, 24, 3712. [CrossRef]

47. Revuelta, J.; Cicchi, S.; Goti, A.; Brandi, A. Enantiopure Cyclic Nitrones: A Useful Class of Building Blocks for Asymmetric Syntheses. Synthesis 2007, 2007, 485-504. [CrossRef]

48. Murahashi, S.-I.; Imada, Y. Synthesis and Transformations of Nitrones for Organic Synthesis. Chem. Rev. 2019, 119, 4684-4716. [CrossRef]

49. Holzapfel, C.W.; Crous, R. Synthesis and reactions of chiral cyclic nitrones derived from D-ribose. Heterocycles 1998, 48, 1337-1342. [CrossRef]

50. Pillard, C.; Desvergnes, V.; Py, S. Diastereoselective addition of alkynylalanes to carbohydrate-derived nitrones. Tetrahedron Lett. 2007, 48, 6209-6213. [CrossRef]

51. Wang, W.-B.; Huang, M.-H.; Li, Y.-X.; Rui, P.-X.; Hu, X.-G.; Zhang, W.; Su, J.-K.; Zhang, Z.-L.; Zhu, J.-S.; $\mathrm{Xu}, \mathrm{W} .-\mathrm{H} . ;$ et al. A Practical Synthesis of Sugar-derived Cyclic Nitrones: Powerful Synthons for the Synthesis of Iminosugars. Synlett 2010, 3, 488-492.

52. Messire, G.; Massicot, F.; Vallee, A.; Vasse, J.-L.; Behr, J.-B. Aza-Henry Reaction with Nitrones, an UnderExplored Transformation. Eur. J. Org. Chem. 2019, 7, 1659-1668. [CrossRef]

53. Cheng, W.-C.; Wang, J.-H.; Yun, W.-Y.; Li, H.-Y.; Hu, J.-M. Rapid preparation of $(3 R, 4 S, 5 R)$ polyhydroxylated pyrrolidine-based libraries to discover a pharmacological chaperone for treatment of Fabry disease. Eur. J. Med. Chem. 2017, 126, 1-6. [CrossRef] [PubMed]

54. Hu, X.-G.; Bartholomew, B.; Nash, R.J.; Wilson, F.X.; Fleet, G.W.J.; Nakagawa, S.; Kato, A.; Jia, Y.-M.; Well, R.V.; $\mathrm{Yu}, \mathrm{C} .-$ Y. Synthesis and Glycosidase Inhibition of the Enantiomer of (-)-Steviamine, the First Example of a New Class of Indolizidine Alkaloid. Org. Lett. 2010, 12, 2562-2565. [CrossRef] [PubMed] 
55. Li, Y.-X.; Huang, M.-H.; Yamashita, Y.; Kato, A.; Jia, Y.-M.; Wang, W.-B.; Fleet, G.W.J.; Nash, R.J.; Yu, C.-Y. L-DMDP, L-homoDMDP and their C-3 fluorinated derivatives: Synthesis and glycosidase-inhibition. Org. Biomol. Chem. 2011, 9, 3405-3414. [CrossRef]

56. Tice, C.M.; Ganem, B. The chemistry of naturally occurring polyamines. 6. Efficient syntheses of N1- and N8-acetylspermidine. J. Org. Chem. 1983, 48, 2106-2108. [CrossRef]

57. Welter, A.; Jadot, J.; Dardenne, G.; Marlier, M.; Casimir, J. 2,5-Dihydroxymethyl 3,4-dihydroxypyrrolidine dans les feuilles de Derris elliptica. Phytochemistry 1976, 15, 747-749. [CrossRef]

58. Kessler, M.; Acuto, O.; Storelli, C.; Murer, H.; Semenza, G.A. A modified procedure for the rapid preparation of efficiently transporting vesicles from small intestinal brush border membranes. Their use in investigating some properties of d-glucose and choline transport systems. Biochim. Biophys. Acta 1978, 506, 136-154. [CrossRef]

59. Ayers, B.J.; Ngo, N.; Jenkinson, S.F.; Martínez, R.F.; Shimada, Y.; Adachi, I.; Weymouth-Wilson, A.C.; Kato, A.; Fleet, G.W.J. Glycosidase Inhibition by All 10 Stereoisomeric 2,5-Dideoxy-2,5-iminohexitols Prepared from the Enantiomers of Glucuronolactone. J. Org. Chem. 2012, 77, 7777-7792. [CrossRef]

60. Cheng, T.-J.R.; Chan, T.-H.; Tsou, E.-L.; Chang, S.-Y.; Yun, W.-Y.; Yang, P.-J.; Wu, Y.-T.; Cheng, W.-C. From Natural Product-Inspired Pyrrolidine Scaffolds to the Development of New Human Golgi $\alpha$-Mannosidase II Inhibitors. Chem. Asian J. 2013, 8, 2600-2604. [CrossRef]

61. Cheng, W.-C.; Wang, J.-H.; Li, H.-Y.; Lu, S.-J.; Hu, J.-M.; Yun, W.-Y.; Chiu, C.-H.; Yang, W.-B.; Chien, Y.-H.; $\mathrm{Hwu}$, W.-L. Bioevaluation of sixteen ADMDP stereoisomers toward alpha-galactosidase A: Development of a new pharmacological chaperone for the treatment of Fabry disease and potential enhancement of enzyme replacement therapy efficiency. Eur. J. Med. Chem. 2016, 123, 14-20. [CrossRef]

62. Takebayashi, M.; Hiranuma, S.; Kanie, Y.; Kajimoto, T.; Kanie, O.; Wong, C.H. A Versatile Synthetic Strategy for the Preparation and Discovery of New Iminocyclitols as Inhibitors of Glycosidases. J. Org. Chem. 1999, 64, 5280-5291. [CrossRef]

63. Kang, S.H.; Ryu, D.H. Intramolecular cyclization of C2 symmetric and meso-iodo amino alcohols: A synthetic approach to azasugars. Tetrahedron Lett. 1997, 38, 607-610. [CrossRef]

64. Ganesan, M.; Madhukarrao, R.V.; Ramesh, N.G. Design and synthesis of new amino-modified iminocyclitols: Selective inhibitors of $\alpha$-galactosidase. Org. Biomol. Chem. 2010, 8, 1527-1530. [CrossRef] [PubMed]

Sample Availability: Samples of the compounds are not available from the authors.

(C) 2020 by the authors. Licensee MDPI, Basel, Switzerland. This article is an open access article distributed under the terms and conditions of the Creative Commons Attribution (CC BY) license (http://creativecommons.org/licenses/by/4.0/). 